\title{
Gradient flow structure and exponential decay of the sandwiched Rényi divergence for primitive Lindblad equations with GNS-detailed balance
}

\author{
Yu Cao, ${ }^{1, *}$ Jianfeng $\mathrm{Lu},{ }^{1,2, \dagger}$ and Yulong $\mathrm{Lu}^{1, \ddagger}$ \\ ${ }^{1}$ Department of Mathematics, Duke University, Box 90320, Durham, NC 27ro8, USA \\ ${ }^{2}$ Department of Physics and Department of Chemistry, \\ Duke University, Box 90320, Durham, NC 27r08, USA
}

(Dated: April 9, 2019)

\begin{abstract}
We study the entropy production of the sandwiched Rényi divergence under the primitive Lindblad equation with GNS-detailed balance. We prove that the Lindblad equation can be identified as the gradient flow of the sandwiched Rényi divergence of any order $\alpha \in(0, \infty)$. This extends a previous result by Carlen and Maas [Journal of Functional Analysis, 273(5), 1810-1869] for the quantum relative entropy (i.e., $\alpha=1$ ). Moreover, we show that the sandwiched Rényi divergence of any order $\alpha \in(0, \infty)$ decays exponentially fast under the time-evolution of such a Lindblad equation.

Keywords: gradient flow dynamics; exponential convergence; sandwiched Rényi divergence; Lindblad equation.
\end{abstract}

\section{INTRODUCTION AND MAIN RESULT}

The characterization of dynamics for open classical and quantum systems is an essential and fundamental topic. One approach to characterize the dynamics for open systems is entropy production [1-6], from an information theory perspective. For Markovian dynamics, the entropy production is positive, which relates to the data processing inequality [7-9] and complies with the second law of thermodynamics $[10,11]$. The entropy production is also related with the gradient flow structure on well-chosen Riemannian manifolds for those Markov semigroups [12-19]. Furthermore, the validity of log-Sobolev inequalities [20-26] helps to prove the exponential decay of those entropies under respective Markov semigroups.

In this paper, we focus on the entropy production of sandwiched Rényi divergences, under primitive Lindblad equations with GNS-detailed balance, for finite dimensional quantum systems. Let $n$ be the dimension of the quantum system. The Lindblad equation is an ordinary differential equation (ODE) $\dot{\rho}_{t}=\mathcal{L}^{\dagger}\left(\rho_{t}\right)$, where $\rho_{t}$ are $n$-by- $n$ density matrices, and the Lindblad super-operator $\mathcal{L}^{\dagger}$ is a linear operator acting on the space of density matrices. The Lindblad equation with GNS-detailed balance (see Theorem II.1 below) has the form

$$
\dot{\rho}_{t}=\mathcal{L}^{\dagger}\left(\rho_{t}\right)=\sum_{j=1}^{J} e^{-\omega_{j} / 2}\left(\left[V_{j} \rho_{t}, V_{j}^{*}\right]+\left[V_{j}, \rho_{t} V_{j}^{*}\right]\right),
$$

where the positive integer $\mathrm{J} \leq n^{2}-1 ; \omega_{j} \in \mathbb{R} ; V_{j}$ are $n$-by- $n$ matrices; constraints for $\omega_{j}$ and $V_{j}$ will be given in Theorem II.1 below; the commutator of matrices $A$ and $B$ is defined as $[A, B]:=$ $A B-B A$. The notion GNS-detailed balance will be explained in Sec. II B below. The Lindblad

\footnotetext{
*yucao@math.duke.edu

† jianfeng@math.duke.edu

$\ddagger$ yulonglu@math.duke.edu
} 
equation is called primitive (also sometimes referred as ergodic, e.g., see [18, 27]) if $\operatorname{Ker}(\mathcal{L})$ is spanned by $\mathbb{I}$, which is the $n$-by- $n$ identity matrix.

Before delving more into Lindblad equations for open quantum systems, we would like to review some existing results of Fokker-Planck equations for open classical systems, for the purpose of comparison. In fact, almost all the conclusions in this paper are motivated by the classical results for Fokker-Planck equations. Let us consider, for instance, a $n$-dimensional Brownian particle under an external potential $\Psi(x)$ with $x \in \mathbb{R}^{n}$. Then the time evolution of the probability density function $p_{t}(x)$ of the Brownian particle is described by the Fokker-Planck equation

$$
\partial_{t} p_{t}(x)=\operatorname{div}\left(p_{t}(x) \nabla \Psi(x)+\nabla p_{t}(x)\right) .
$$

It has been shown by Jordan, Kinderlehrer, and Otto [12] that such types of Fokker-Planck equations can be viewed as the Wasserstein gradient flow dynamics of the relative entropy (or the free energy functional). Moreover, based on the method of entropy production and the log-Sobolev inequality [23], one can prove that the solution of the Fokker-Planck equation converges to the Gibbs (stationary) distribution exponentially fast in the relative entropy. In our recent work [28], we generalized these results to the classical Rényi divergence of any order $\alpha \in(0, \infty)$ : (1) the same Fokker-Planck equation can be formally identified as the gradient flow dynamics of the classical Rényi divergence of any order $\alpha$ with respect to a transportation distance defined by a certain metric tensor; (2) the solution of the Fokker-Planck equation converges exponentially fast to the Gibbs distribution in the classical Rényi divergence. Apart from Fokker-Planck equations, several other dissipative dynamics can also be viewed as the gradient flow dynamics of various transport metrics, for instance, the heat equation [14], finite Markov Chains [15] and porous medium equations $[13,16]$.

As for open quantum systems, Lindblad equations [29, 30], generally regarded as the quantum analog of Fokker-Planck equations, could be derived from the weak-coupling limit of the system and baths $[31,32]$. Due to the similar role that Lindblad and Fokker-Planck equations play for open quantum and classical systems respectively, it is interesting to study Lindblad equations from the perspective of gradient flows. In a previous work by Carlen and Maas, they showed that fermionic Fokker-Planck equation can be identified as the gradient flow dynamics of the quantum relative entropy [17]; in their more recent work, they extended results from [17] and showed that for finite dimensional quantum systems, primitive Lindblad equations with GNS-detailed balance can indeed be identified as the gradient flow dynamics of the quantum relative entropy [18]; at almost the same time when the first preprint of this work appeared, they also established a unified picture of gradient flows including the previous two cases and Markov Chains in the setting of $\mathrm{C}^{*}$-algebras [19]. The generalization of their results from finite dimensional matrices to infinite dimensional noncommutative algebras has been recently considered in [33]. In the present paper, we focus on the finite dimensional setting and aim to characterize the gradient flow structure of Lindblad equations in terms of a family of quantum divergences - sandwiched Rényi divergences [34, 35], including the quantum relative entropy as a specific instance. Moreover, we are also interested in quantifying the decaying property of the solution $\rho_{t}$ of the Lindblad equation (1) in terms of the sandwiched Rényi divergence.

The sandwiched Rényi divergence is defined as follows.

Definition I.1 (Sandwiched Rényi divergence [34]). Given two positive semi-definite matrices $\rho, \sigma$ such that $\rho \ll \sigma$ and $\rho \neq 0$, the sandwiched Rényi divergence $D_{\alpha}(\rho \| \sigma)$ of order $\alpha \in(0, \infty)$ is 
defined by

$$
D_{\alpha}(\rho \| \sigma):=\left\{\begin{array}{cl}
\frac{1}{\alpha-1} \log \left(\operatorname{Tr}\left[\left(\sigma^{\frac{1-\alpha}{2 \alpha}} \rho \sigma^{\frac{1-\alpha}{2 \alpha}}\right)^{\alpha}\right]\right), & \alpha \in(0,1) \cup(1, \infty) ; \\
\operatorname{Tr}(\rho \log (\rho)-\rho \log (\sigma)), & \alpha=1 .
\end{array}\right.
$$

The notation $\rho \ll \sigma$ means the kernel of $\sigma$ is a subspace of the kernel of $\rho$. The term $\operatorname{Tr}(\rho)$ in [34, Definition 2] is simply set as one, since we only consider $\rho$ to be a density matrix in this paper. $\sigma$ is assumed to be a full-rank density matrix, so $\rho \ll \sigma$ is always satisfied for this paper. For any fixed $\rho$ and full-rank $\sigma$, the sandwiched Rényi divergence is continuous with respect to the order $\alpha$, in particular, $D(\rho \| \sigma)=\lim _{\alpha \rightarrow 1} D_{\alpha}(\rho \| \sigma)$.

The sandwiched Rényi divergence is a quantum analog of the classical Rényi divergence, and has recently received much attention due to its use in quantum information theory; see e.g., [35, 36]. The sandwiched Rényi divergence unifies many entropy measures: when $\alpha=1$, it reduces to the quantum relative entropy; when $\alpha \rightarrow \infty$, it converges to the quantum relative max-entropy; when $\alpha=1 / 2$ and $\alpha=2$, it is closely connected to the quantum fidelity and $\chi^{2}$ divergence respectively [37]. The sandwiched Rényi divergence satisfies the data processing inequality for any order $\alpha \geq \frac{1}{2}$ and for any completely positive trace-preserving (CPTP) map [8, 34, 38]. For fixed density matrices $\rho$ and $\sigma$, it is monotonically increasing with respect to the order $\alpha \in(0, \infty)[34$, Theorem 7]. The sandwiched Rényi divergence has also been used to study the quantum second laws of thermodynamics [11], which is a major motivation of our work. Its global convergence rate and mixing time under quantum Markov semigroups has been studied in [39].

We remark that there is another well-studied family of quantum Rényi divergences known as Petz-Rényi divergence [40], which is defined by $\widetilde{D}_{\alpha}(\rho \| \sigma):=\frac{1}{\alpha-1} \log \left(\operatorname{Tr}\left(\rho^{\alpha} \sigma^{1-\alpha}\right)\right)$. When $\rho$ and $\sigma$ commute, the sandwiched Rényi divergence is identical to the Petz-Rényi divergence. We also remark that [41] has studied more generalized $(\alpha, z)$-quantum Rényi divergences, which include sandwiched Rényi divergences and Petz-Rényi divergences as special instances; the discussion on the data processing inequality of $(\alpha, z)$-quantum Rényi divergences can be found in a recent review paper [42] and the references therein.

The first main result of this paper is the following theorem.

Theorem I.2. Consider a primitive Lindblad equation with GNS-detailed balance (1) and suppose its unique stationary state $\sigma$ is full-rank. For any order $\alpha \in(0, \infty)$, consider the energy functional $D_{\alpha}(\rho \| \sigma)$ with respect to $\rho$. In the space of strictly positive density matrices $\mathfrak{D}_{+}$equipped with the metric tensor defined in the Definition III.6, the gradient flow dynamics of the sandwiched Rényi divergence $D_{\alpha}(\rho \| \sigma)$ is exactly the Lindblad equation (1).

This theorem immediately implies the monotonicity of the sandwiched Rényi divergence under the evolution of such Lindblad equations, summarized in the following Corollary I.3. For $\alpha \geq \frac{1}{2}$, this corollary can be proved simply by applying the data processing inequality [8]. In our case, the monotonicity also holds for $\alpha \in\left(0, \frac{1}{2}\right)$ for primitive Lindblad equations with GNS-detailed balance, though the data processing inequality does not generally hold for sandwiched Rényi divergences when $\alpha<\frac{1}{2}$ [43, Sec. 4].

Corollary I.3. Under the same conditions as in Theorem I.2, suppose $\rho .:[0, \infty) \rightarrow \mathfrak{D}_{+}$is the solution of that Lindblad equation. For any $\alpha \in(0, \infty)$, the sandwiched Rényi divergence $D_{\alpha}\left(\rho_{t} \| \sigma\right)$, is non-increasing with respect to the time $t$, i.e. $\partial_{t} D_{\alpha}\left(\rho_{t} \| \sigma\right) \leq 0$.

Theorem I.2 only provides a sufficient detailed balance condition for Lindblad equations to be the gradient flow dynamics of sandwiched Rényi divergences. Later in Sec. IV, we will discuss 
in Theorem IV.1 the necessary condition for Lindblad equations to be possibly expressed as the gradient flow dynamics of sandwiched Rényi divergences, by adapting and extending the analysis in [19, Theorem 2.9]. Loosely speaking, the family of Lindblad equations that can be possibly written as the gradient flow dynamics of sandwiched Rényi divergences of any order $\alpha \in(0, \infty)$ is not substantially larger than the class of Lindblad equations with GNS-detailed balance, which explains the reason why we restrict our attention to Lindblad equations with GNS-detailed balance in Theorem I.2. However, identifying a sufficient and necessary condition still remains open.

Next, we would like to study the exponential decay of sandwiched Rényi divergences. For FokkerPlanck equations in classical systems, the exponential decay of the relative entropy could be proved via the (classical) log-Sobolev inequality, namely, the relative entropy is bounded above by the relative Fisher information. This approach has been generalized in our previous work [28] to obtain the exponential decay of the classical Rényi divergence by introducing the relative $\alpha$-Fisher information. For quantum systems, in the same flavor, let us first define the quantum relative $\alpha$-Fisher information as follows.

Definition I.4. Suppose $\mathcal{L}^{\dagger}$ is the generator of a primitive Lindblad equation with GNS-detailed balance and the unique stationary state $\sigma$ is full-rank. The quantum relative $\alpha$-Fisher information between $\rho \in \mathfrak{D}_{+}$and $\sigma$ is defined as

$$
\mathscr{I}_{\alpha}(\rho \| \sigma):= \begin{cases}-\left\langle\frac{\delta D_{\alpha}(\rho \| \sigma)}{\delta \rho}, \mathcal{L}^{\dagger}(\rho)\right\rangle, & \text { if } \alpha \neq 1 \\ -\left\langle\log (\rho)-\log (\sigma), \mathcal{L}^{\dagger}(\rho)\right\rangle, & \text { if } \alpha=1 .\end{cases}
$$

When $\alpha=1$, we denote $\mathscr{I}(\rho \| \sigma) \equiv \mathscr{I}_{1}(\rho \| \sigma)$ and call it quantum relative Fisher information for simplicity. The inner product $\langle\cdot, \cdot\rangle$ herein is the Hilbert-Schmidt inner product, defined by $\langle A, B\rangle:=\operatorname{Tr}\left(A^{*} B\right)$ for all matrices $A, B$. Notice that in the definition above, the quantum relative $\alpha$ Fisher information depends on the Lindblad super-operator $\mathcal{L}^{\dagger}$, which is different from the classical setting where the relative $\alpha$-Fisher information only depends on the Gibbs stationary distribution (see [28]). Also, observe that if $\rho_{t}$ solves the Lindblad equation (1), then $\mathscr{I}_{\alpha}\left(\rho_{t} \| \sigma\right)=-\partial_{t} D_{\alpha}\left(\rho_{t} \| \sigma\right)$. Since by Corollary I.3 $\partial_{t} D_{\alpha}\left(\rho_{t} \| \sigma\right) \leq 0$, the quantum relative $\alpha$-Fisher information $\mathscr{I}_{\alpha}\left(\rho_{t} \| \sigma\right)$ is non-negative.

Definition I.5. Suppose the density matrix $\sigma$ is the unique stationary state of the Lindblad equation (1). For a fixed order $\alpha \in(0, \infty), \sigma$ is said to satisfy the quantum $\alpha$-log Sobolev inequality (or quantum $\alpha$-LSI in short) if there exists $K_{\alpha}>0$, such that

$$
D_{\alpha}(\rho \| \sigma) \leq \frac{1}{2 K_{\alpha}} \mathscr{I}_{\alpha}(\rho \| \sigma), \quad \forall \rho \in \mathfrak{D},
$$

where $\mathfrak{D}$ is the space of density matrices. $K_{\alpha}$ is called the $\alpha$-log Sobolev constant. When $\alpha=1$, we call (5) quantum log-Sobolev inequality and denote $K \equiv K_{1}$ for simplicity.

The $\alpha$-log Sobolev constant $K_{\alpha}$ has been considered in [39, Definition 3.1]. When $\alpha=1$, the above form reduces to the quantum log-Sobolev inequality in [18, Eq. (8.17)].

Remark I.6. Another definition of quantum $\alpha$-LSI was given previously under the framework of noncommutative $\mathbb{L}_{\alpha}$ spaces (see e.g., [44, Definition 3.5], [45, Definition 11] and [46, Sec. 2.5]). We will briefly revisit this concept in Sec. II E and we shall denote the log-Sobolev constant in this setting up by $\kappa_{\alpha}$. The essential difference is that for the definition of $\kappa_{\alpha}$, the entropy function uses the quantum relative entropy for a density matrix raising to the power of $\alpha$, instead of using sandwiched Rényi divergence (see (30) for more details). 
For Lindblad equations with GNS-detailed balance, the primitivity of Lindblad equations is equivalent to the validity of the quantum LSI, summarized in the following Proposition I.7. It is important to note that by Lemma II.4 below, if the Lindblad equation satisfies GNS-detailed balance, then $-\mathcal{L}$ is a positive semi-definite operator, with respect to the inner product $\langle\cdot, \cdot\rangle_{1 / 2}$ which is defined by $\langle A, B\rangle_{1 / 2}:=\operatorname{Tr}\left(A^{*} \sigma^{1 / 2} B \sigma^{1 / 2}\right)$ for all matrices $A, B$. As a consequence, primitivity of $\mathcal{L}$ implies that $-\mathcal{L}$ has a positive spectral gap, denoted by $\lambda_{\mathcal{L}}$. The proposition below provides two-side estimates of $K$ in terms of $\lambda_{\mathcal{L}}$.

Proposition I.7. Consider the Lindblad equation with GNS-detailed balance (1) and suppose a full-rank density matrix $\sigma$ is its stationary state. Then, $\sigma$ satisfies the quantum LSI iff the Lindblad equation is primitive. More quantitatively, if the Lindblad equation is primitive, we have

$$
\frac{1}{1-\log \left(\sqrt{\lambda_{\min }(\sigma)}\right)} \lambda_{\mathcal{L}} \leq K \leq \lambda_{\mathcal{L}}
$$

where $\lambda_{\min }(\sigma)$ is the smallest eigenvalue of $\sigma$.

Proposition I.7 follows mainly from [39, Theorem 5.3], [45, Theorem 16] (or see [39, Theorem 4.1]), and the quantum Stroock-Varopoulos inequality [46, Corollary 16]. The proof is postponed to Sec. V C. We remark that the lower bound above is not sharp and it remains an interesting question to find a tighter lower bound.

It is not hard to see that if the quantum $\alpha$-LSI (5) is valid, then it follows immediately from Grönwall inequality that (see also [39, Theorem 3.2])

$$
D_{\alpha}\left(\rho_{t} \| \sigma\right) \leq D_{\alpha}\left(\rho_{0} \| \sigma\right) e^{-2 K_{\alpha} t}, \quad \forall t \geq 0 .
$$

The validity of (5) with a positive $K_{\alpha}$ is in general difficult to establish. For depolarizing semigroup, $K_{2}$ has been computed in [39, Theorem 3.3] and $K$ has been studied in [47]. For the depolarizing semigroup with stationary state $\sigma$ being the maximally mixed state, both upper bound and lower bound of $K_{\alpha}$ with $\alpha \geq 2$ have been provided in [39, Theorem 3.4]. Moreover, it has been proved in [39, Theorem 4.1] that the $\alpha$-log Sobolev constant cannot exceed the spectral gap, namely for any $\alpha \geq 1, K_{\alpha} \leq \lambda_{\mathcal{L}}$. In the present paper, instead of pursuing the global decay of the sandwiched Rényi divergence $D_{\alpha}\left(\rho_{t} \| \sigma\right)$ as in (6), we focus on the exponential decay of $D_{\alpha}\left(\rho_{t} \| \sigma\right)$ in the large time regime. More specifically, our second main result (Theorem I.8) shows that the sandwiched Rényi divergence $D_{\alpha}\left(\rho_{t} \| \sigma\right)$ decays exponentially with the sharp rate $2 \lambda_{\mathcal{L}}$ when $t$ is large.

Theorem I.8. Under the same conditions as in Theorem I.2, for any given solution $\rho .:[0, \infty) \rightarrow$ $\mathfrak{D}_{+}$of the Lindblad equation and any $\epsilon \in\left(0, \frac{\lambda_{\min }(\sigma)^{2}}{2}\right)$, there exist $C_{\alpha, \epsilon}$ and $\tau_{\alpha, \epsilon}$ such that

$$
D_{\alpha}\left(\rho_{t} \| \sigma\right) \leq C_{\alpha, \epsilon} D_{\alpha}\left(\rho_{0} \| \sigma\right) e^{-2 \lambda_{\mathcal{L}} t}, \quad \forall t \geq \tau_{\alpha, \epsilon},
$$

where

$$
C_{\alpha, \epsilon}=\frac{e^{D_{2}\left(\rho_{0} \| \sigma\right)}-1}{D_{\alpha}\left(\rho_{0} \| \sigma\right)} \exp \left(\Theta(\alpha-2) 2 \lambda_{\mathcal{L}} T_{\alpha, \epsilon}\right)
$$

and

$$
\tau_{\alpha, \epsilon}= \begin{cases}0, & \alpha \leq 2 \\ T_{\alpha, \epsilon}+\max \left(0, \frac{1}{2 K} \log \left(D\left(\rho_{0} \| \sigma\right) / \epsilon\right)\right), & \alpha>2 .\end{cases}
$$


In the above, $\lambda_{\min }(\sigma)$ is the smallest eigenvalue of $\sigma ; T_{\alpha, \epsilon}=\frac{1}{2 K \eta} \log (\alpha-1)$ is a function depending on $\alpha$ and $\epsilon ; \Theta$ is the Heaviside function;

$$
\begin{aligned}
& \eta=\min \left(\frac{1}{2}, \min _{j=1}^{J}\left\{\frac{2 \sqrt{e^{\omega_{j}} \frac{1}{\Lambda}}}{1+e^{\omega_{j}} \Lambda}\right\}\right), \\
& \Lambda=\frac{\lambda_{\max }(\sigma)}{\lambda_{\min }(\sigma)} \exp \left(2 \sqrt{2 \epsilon} \frac{2 \lambda_{\min }(\sigma)-\sqrt{2 \epsilon}}{\lambda_{\min }(\sigma)\left(\lambda_{\min }(\sigma)-\sqrt{2 \epsilon}\right)}\right),
\end{aligned}
$$

where $\omega_{j}$ are Bohr frequencies determined by the stationary state $\sigma$ (see Sec. II B) and $\lambda_{\max }(\sigma)$ is the largest eigenvalue of $\sigma$.

The proof of Theorem I.8 can be found in Sec. V. Here we sketch the strategies used in the proof. First, we prove this theorem for the case $\alpha=2$ by using a uniform lower bound of $\mathscr{I}_{2}(\rho \| \sigma)$ (see (58)). Next, we prove that the quantum LSI (5) holds (see Proposition I.7). Based on the quantum LSI (see (5) for $\alpha=1$ ), we prove a quantum comparison theorem (see Proposition V.8), which implies the exponential decay of the sandwiched Rényi divergence of any order $\alpha \in(0, \infty)$ (see Proposition V.10).

Some remarks of this theorem are in order:

Remark I.9. 1) The upper bounds for $\tau_{\alpha, \epsilon}$ and $C_{\alpha, \epsilon}$ may not be sharp; a better bound might be important if one wants to study the mixing time for quantum decoherence, which however will not be pursued here. For fixed $\alpha>2$, when $\epsilon$ becomes smaller, $T_{\alpha, \epsilon}$ decreases and we have a smaller prefactor $C_{\alpha, \epsilon}$, at the expense of waiting longer time $\tau_{\alpha, \epsilon}$ since the term $D\left(\rho_{0} \| \sigma\right) / \epsilon$ blows up as $\epsilon \downarrow 0^{+}$.

2) The artificial $\epsilon$ and the complicated expression of $T_{\alpha, \epsilon}$ come from Proposition V.8. There is a different way to quantify $\eta$ under the assumption of the quantum 2-LSI in the sense of noncommutative $\mathbb{L}_{\alpha}$ spaces; please see Sec. II E for background and the discussion in Sec. VF for more details. We prefer this version because the log-Sobolev inequality for $\alpha=1$ is also used for the classical result in [28, Theorem 1.2]. One could remove the dependence of the parameter $\epsilon$ by simply choosing, e.g., $\epsilon=\lambda_{\min }(\sigma)^{2} / 8$ in this theorem, then

$$
\Lambda=\frac{\lambda_{\max }(\sigma)}{\lambda_{\min }(\sigma)} e^{3}, \quad \eta=\min \left(\frac{1}{2}, \min _{j=1}^{\jmath}\left\{\frac{2 e^{-3 / 2} \sqrt{e^{\omega_{j}} \frac{\lambda_{\min }(\sigma)}{\lambda_{\max }(\sigma)}}}{1+e^{\omega_{j}} \frac{\lambda_{\max }(\sigma)}{\lambda_{\min }(\sigma)} e^{3}}\right\}\right) .
$$

If we further assume that $\sigma$ is the maximally mixed state, i.e., $\sigma=\frac{1}{n} \mathbb{I}$, then

$$
\Lambda=e^{3}, \quad \eta=\frac{2 e^{-3 / 2}}{1+e^{3}}
$$

are independent of the dimension $n$.

Our contribution. We summarize here contributions of the current work.

1) We extend the work of Carlen and Maas in [18] by proving that primitive Lindblad equations with GNS-detailed balance can be identified as the gradient flow dynamics of the sandwiched Rényi divergence of any order $\alpha \in(0, \infty)$; see Theorem I.2. In addition, we follow a recent work of Carlen and Maas [19] to study the necessary condition for Lindblad equations to be possibly written as the gradient flow dynamics of sandwiched Rényi divergences; see Theorem IV.1. 
2) We prove that the sandwiched Rényi divergence decays exponentially fast under the evolution of primitive Lindblad equations with GNS-detailed balance; see Theorem I.8. Our result (7) shows that the sandwiched Rényi divergence decays with an asymptotic rate $2 \lambda_{\mathcal{L}}$, which is sharp according to [39, Theorem 4.1].

To prove Theorem I.8, we first prove a quantum comparison theorem (see Proposition V.8), which is of interest in its own right. It is essentially a hypercontractivity-type estimation of the solution of the Lindblad equation, enabling us to bound $D_{\alpha_{1}}\left(\rho_{T} \| \sigma\right)$ by $D_{\alpha_{0}}\left(\rho_{0} \| \sigma\right)$ with some $T>0$ when $\alpha_{1}>\alpha_{0}$. As a direct consequence of the comparison theorem, Proposition V.10 shows that the sandwiched Rényi divergence exponentially decays for all orders $\alpha \in(0, \infty)$. Such a comparison result for classical Rényi divergences was proved for the Fokker-Planck equation in [28, Theorem 1.2]. For quantum dynamics, a similar comparison result was also obtained in [46, Corollary 17]. However, a major difference between our result and theirs is that we use $K$ in the estimation of the waiting time, while they use $\kappa_{2}$ (see (28)). See a detailed discussion in Sec. VF.

The rest of the paper is organized as follows. In Sec. II, we will recall several preliminary results on, e.g., gradient flows, Lindblad equations with detailed balance, and noncommutative $\mathbb{L}_{\alpha}$ spaces. Sec. III will be devoted to the proof of Theorem I.2 and Corollary I.3. In Sec. IV, we will study the necessary detailed balance condition for Lindblad equations to be the gradient flow dynamics of the sandwiched Rényi divergence of order $\alpha \in(0, \infty)$; see Theorem IV.1. Proposition I.7 and Theorem I.8 will be proved in Sec. V. Finally, we will discuss in Sec. VI some potential directions in the future, in particular, the generalization of quantum Wasserstein distance involving the sandwiched Rényi divergence.

\section{PRELIMINARIES}

In this section, we recall some technical notions: gradient flow dynamics on the Riemannian manifold, Lindblad equations with GNS-detailed balance, quantum analog of gradient and divergence operators, chain rule identity, and quantum noncommutative $\mathbb{L}_{\alpha}$ spaces. Most results from Sec. II B to Sec. II D follow from [18]; Sec. II E is mostly based on [46].

Notations: Throughout this section and the rest of this paper, we will use the following notations. Denote $\mathfrak{M}$ as the space of all $n \times n$ complex-valued matrices. The space of Hermitian matrices is $\mathfrak{A}$; the space of strictly positive matrices is $\mathfrak{P}_{+}$; the space of traceless Hermitian matrices is $\mathfrak{A}_{0}$. The space of density matrices is $\mathfrak{D}$ and the space of invertible density matrices is $\mathfrak{D}_{+}$. Unity $\mathbb{I}$ is the $n$-by- $n$ identity matrix; $\mathcal{I}$ is the identity super-operator on $\mathfrak{M}$.

Hilbert-Schmidt inner product is $\langle A, B\rangle:=\operatorname{Tr}\left(A^{*} B\right)$ for $A, B \in \mathfrak{M}$. We extend the inner product $\langle\cdot, \cdot\rangle$ to vector fields over $\mathfrak{M}$ by $\langle\vec{A}, \vec{B}\rangle:=\sum_{j=1}^{J}\left\langle A_{j}, B_{j}\right\rangle$, where $\vec{A}=\left(\begin{array}{lll}A_{1} & A_{2} \cdots A_{\mathrm{J}}\end{array}\right)$, $\vec{B}=\left(\begin{array}{llll}B_{1} & B_{2} & \cdots & B_{\mathrm{J}}\end{array}\right)$ and $A_{j}, B_{j} \in \mathfrak{M}$ for all $1 \leq j \leq \mathrm{J}$; here $\mathrm{J}$ is a fixed positive integer. The space of such vector fields is denoted by $\mathfrak{M}^{\mathrm{J}}$. Such a convention is also applied analogously to all inner products on $\mathfrak{M}$.

Denote $\lambda_{\min }(\rho)$ as the smallest eigenvalue of a density matrix $\rho$ and $\lambda_{\max }(\rho)$ as the largest one. Asterisk $*$ in the superscript means Hermitian-conjugate; dagger $\dagger$ means the adjoint operator with respect to the Hilbert-Schmidt inner product.

To simplify the notation, a weight operator $\Gamma_{\sigma}: \mathfrak{M} \rightarrow \mathfrak{M}$ is defined by

$$
\Gamma_{\sigma}(A):=\sigma^{\frac{1}{2}} A \sigma^{\frac{1}{2}}
$$


for any matrix $A \in \mathfrak{M}$. Hence, for any $\gamma \in \mathbb{R}$,

$$
\Gamma_{\sigma}^{\gamma}(A)=\sigma^{\gamma / 2} A \sigma^{\gamma / 2} .
$$

\section{A. Gradient flow dynamics}

Consider a Riemannian manifold $\left(\mathcal{M}, g_{\rho}\right): \mathcal{M}$ is a smooth manifold and $g_{\rho}(\cdot, \cdot)$ is an inner product on the tangent space $\mathcal{T}_{\rho} \mathcal{M}$ for any $\rho \in \mathcal{M}$. Given any (energy) functional $E$ on $\mathcal{M}$, the gradient, denoted by $\left.\operatorname{grad} E\right|_{\rho}$ at any state $\rho \in \mathcal{M}$, is defined as the element in the tangent space $\mathcal{T}_{\rho} \mathcal{M}$ such that

$$
g_{\rho}\left(\left.\operatorname{grad} E\right|_{\rho}, \nu\right)=\left.\frac{\mathrm{d}}{\mathrm{d} \epsilon} E(\rho+\epsilon \nu)\right|_{\epsilon=0}, \quad \forall \nu \in \mathcal{T}_{\rho} \mathcal{M}
$$

Then, the gradient flow dynamics refers to following autonomous differential equation

$$
\frac{\mathrm{d}}{\mathrm{d} t} \rho_{t}=-\left.\operatorname{grad} E\right|_{\rho_{t}} .
$$

This gradient flow dynamics is essentially a generalization of steepest descend dynamics in the Euclidean space. In this paper, we will take $\mathcal{M}$ to be the space of strictly positive density matrices $\mathfrak{D}_{+}$. The choice of $g_{\rho}$ is more subtle and technical; thus we will explain it below in Sec. III.

\section{B. Quantum Markov semigroup with GNS-detailed balance}

For a continuous time-parameterized semigroup $\left(\mathcal{P}_{t}\right)_{t \geq 0}$, acting on the space of linear operators on a finite dimensional Hilbert space $\mathbb{C}^{n}$, it is called a quantum Markov semigroup (QMS) if $\mathcal{P}_{t}$ is completely positive and $\mathcal{P}_{t}(\mathbb{I})=\mathbb{I}$, for any $t \geq 0$. The generator of the QMS is denoted by $\mathcal{L}$, i.e., $\mathcal{P}_{t}=e^{t \mathcal{L}}$. Physically, this semigroup $\mathcal{P}_{t}$ usually refers to the time evolution super-operator for observables in the Heisenberg picture.

To explain quantum detailed balance, let us define inner products $\langle\cdot, \cdot\rangle_{s}$ parameterized by $s \in$ $[0,1]$, for a given full-rank density matrix $\sigma$, in the following way: for any $A, B \in \mathfrak{M}$,

$$
\langle A, B\rangle_{s}:=\operatorname{Tr}\left(\sigma^{s} A^{*} \sigma^{1-s} B\right) .
$$

Essentially, $\langle\cdot, \cdot\rangle_{s}$ is a $\sigma$-weighted Hilbert-Schmidt inner product. The QMS is said to satisfy GNSdetailed balance if $\mathcal{P}_{t}$ is self-adjoint with respect to the inner product $\langle\cdot, \cdot\rangle_{1}$ for all $t \geq 0$, that is to say, $\left\langle\mathcal{P}_{t}(A), B\right\rangle_{1}=\left\langle A, \mathcal{P}_{t}(B)\right\rangle_{1}$ for all $A, B \in \mathfrak{M}$ and $t \geq 0$. In some literature, the case $s=1 / 2$ is considered instead and the quantum Markov semigroup is said to satisfy KMS-detailed balance if $\mathcal{P}_{t}$ is self-adjoint with respect to the inner product $\langle\cdot, \cdot\rangle_{1 / 2}$. It is not hard to verify that the QMS satisfies KMS-detailed balance iff

$$
\Gamma_{\sigma}^{-1} \circ \mathcal{L}^{\dagger} \circ \Gamma_{\sigma}=\mathcal{L} .
$$

An important relation is that GNS-detailed balance is a more restrictive situation and it implies KMS-detailed balance; reversely, it is not true. More discussion on the relation between GNS and KMS detailed balance could be found in [18], in particular, Theorem 2.9 therein. Another important 
consequence is that if $\mathcal{P}_{t}$ satisfies detailed balance (either KMS or GNS), then $\sigma$ is invariant under dynamical evolution $\mathcal{P}_{t}^{\dagger}$, that is to say, $\mathcal{P}_{t}^{\dagger}(\sigma)=\sigma$. We add the prefix GNS and KMS to explicitly distinguish these two conditions. More results on the quantum detailed balance can be found in e.g. $[37,48-50]$ and the references therein.

The general form of quantum Markov semigroups with GNS-detailed balance is given by the theorem below.

Theorem II.1 ([18, Theorem 3.1]). Suppose that the $Q M S \mathcal{P}_{t}$ satisfies GNS-detailed balance, then

$$
\begin{aligned}
\mathcal{L}(A) & =\sum_{j=1}^{J} e^{-\omega_{j} / 2} V_{j}^{*}\left[A, V_{j}\right]+e^{\omega_{j} / 2}\left[V_{j}, A\right] V_{j}^{*} \\
& =\sum_{j=1}^{J} e^{-\omega_{j} / 2}\left(V_{j}^{*}\left[A, V_{j}\right]+\left[V_{j}^{*}, A\right] V_{j}\right),
\end{aligned}
$$

where $\omega_{j} \in \mathbb{R}$ and $\mathrm{J} \leq n^{2}-1$. Moreover $\left\{V_{j}\right\}_{j=1}^{J}$ satisfy the following:

1. $\operatorname{Tr}\left(V_{j}\right)=0$ and $\left\langle V_{j}, V_{k}\right\rangle=\delta_{j, k} c_{j}$ for all $1 \leq j, k \leq \mathrm{J}$; constants $c_{j}>0$ (for all $1 \leq j \leq \mathrm{J}$ ) come from normalization;

2. for each $j$, there exists $1 \leq j^{\prime} \leq \mathrm{J}$ such that $V_{j}^{*}=V_{j^{\prime}}$;

3. $\Delta_{\sigma}\left(V_{j}\right)=e^{-\omega_{j}} V_{j}$, where the modular operator $\Delta_{\sigma}: \mathfrak{M} \rightarrow \mathfrak{M}$ is defined as

$$
\Delta_{\sigma}(A):=\sigma A \sigma^{-1}, \quad \text { for } A \in \mathfrak{M}
$$

4. if $V_{j}^{*}=V_{j^{\prime}}$ then $c_{j}=c_{j^{\prime}}$ and $\omega_{j}=-\omega_{j^{\prime}}$.

Then it could be easily derived that (see also Eq. (3.7) in [18])

$$
\begin{aligned}
\mathcal{L}^{\dagger}(A) & =\sum_{j=1}^{J}\left(e^{-\omega_{j} / 2}\left[V_{j} A, V_{j}^{*}\right]+e^{\omega_{j} / 2}\left[V_{j}^{*}, A V_{j}\right]\right) \\
& =\sum_{j=1}^{J} e^{-\omega_{j} / 2}\left(\left[V_{j} A, V_{j}^{*}\right]+\left[V_{j}, A V_{j}^{*}\right]\right),
\end{aligned}
$$

which is well known as the Lindblad super-operator.

Example II.2. Consider the depolarizing semigroup with the generator $\mathcal{L}_{\text {depol }}^{\dagger}(A)=\gamma(\sigma \operatorname{Tr}(A)-A)$, and $\gamma>0$. It is not hard to verify that $\mathcal{L}_{\text {depol }}(A)=\gamma(\operatorname{Tr}(\sigma A) \mathbb{I}-A)$ and that $\mathcal{L}_{\text {depol }}$ is self-adjoint with respect to the inner product $\langle\cdot, \cdot\rangle_{1}$. Thus this depolarizing semigroup satisfies the GNS-detailed balance.

The following lemma is a special case of [18, Lemma 2.5] and is enough for our purpose.

Lemma II.3. Suppose $Q M S \mathcal{P}_{t}$ satisfies the GNS-detailed balance, then

$$
\left[\mathcal{P}_{t}, \Delta_{\sigma}\right]=0, \quad \text { and equivalently } \quad\left[\mathcal{L}, \Delta_{\sigma}\right]=0 .
$$




\section{Noncommutative analog of gradient and divergence}

Given those $V_{j}$ from (13), define the noncommutative partial derivative $\partial_{j}$ by

$$
\partial_{j}(A):=\left[V_{j}, A\right],
$$

for any matrix $A \in \mathfrak{M}$; as a result, its adjoint operator $\partial_{j}^{\dagger}$ has the form

$$
\partial_{j}^{\dagger}(A)=\left[V_{j}^{*}, A\right] .
$$

Let $\mathfrak{M}^{\jmath}$ be the vector fields over $\mathfrak{M}$, i.e., each element $\vec{A} \in \mathfrak{M}^{\jmath}$ has the form $\vec{A}=\left(\begin{array}{llll}A_{1} & A_{2} & \cdots & A_{\jmath}\end{array}\right)$ where $A_{j} \in \mathfrak{M}$ for all $1 \leq j \leq \mathrm{J}$. The noncommutative gradient $\nabla: \mathfrak{M} \rightarrow \mathfrak{M}^{\jmath}$ is defined by

$$
\nabla A:=\left(\begin{array}{llll}
\partial_{1} A & \partial_{2} A & \cdots & \partial_{\jmath} A
\end{array}\right) \in \mathfrak{M}^{\mathrm{J}} .
$$

The noncommutative divergence, acting on $\mathfrak{M}^{\lrcorner}$, is defined by

$$
\operatorname{div}(\vec{A}):=-\sum_{j=1}^{J} \partial_{j}^{\dagger}\left(A_{j}\right) \equiv \sum_{j=1}^{J}\left[A_{j}, V_{j}^{*}\right]
$$

for $\vec{A}=\left(\begin{array}{llll}A_{1} & A_{2} & \cdots & A_{J}\end{array}\right) \in \mathfrak{M}^{\mathrm{J}}$. The divergence is defined as this so that it is the adjoint operator of gradient, with a multiplicative factor -1 , analogous to the classical case.

Lemma II.4 ([18, Eq. (5.3)]). For a quantum Markov semigroup with GNS-detailed balance,

$$
\langle\nabla A, \nabla A\rangle_{1 / 2}=\langle A,-\mathcal{L}(A)\rangle_{1 / 2} .
$$

Thus $-\mathcal{L}$ is a positive semi-definite operator on the Hilbert space $\left(\mathfrak{M},\langle\cdot, \cdot\rangle_{1 / 2}\right)$.

Lemma II.5 ([18, Theorem 5.3]). Suppose the Lindblad equation satisfies GNS-detailed balance. Then the kernel of $\mathcal{L}$ equals to the commutant of $\left\{V_{j}\right\}_{j=1}^{J}$. In other words, $\operatorname{Ker}(\mathcal{L})=\operatorname{Ker}(\nabla)$.

The lemma below gives a sufficient condition for Lindblad equations with GNS-detailed balance to be primitive, though the analysis in the rest of this manuscript does not rely on this result. However, the condition $\mathrm{J}=n^{2}-1$ is not necessary for Lindblad equations with GNS-detailed balance to be primitive. For example, the case $n=2, \sigma=\frac{1}{2} \mathbb{I}, V_{1}=\sigma_{X}$ and $V_{2}=\sigma_{Z}$ with $\mathrm{J}=2$ still leads to a primitive Lindblad equation, though $\mathrm{J}<n^{2}-1 ; \sigma_{X}$ and $\sigma_{Z}$ here are Pauli matrices.

Lemma II.6. If the Lindblad equation satisfies the GNS-detailed balance and $\mathrm{J}=n^{2}-1$, then the Lindblad equation is primitive.

Proof. Note that $\left\{\mathbb{I}, V_{1}, V_{2}, \cdots V_{J}\right\}$ form a (non-normalized) basis of $\mathfrak{M}$. Hence for any matrix $A \in \mathfrak{M}$, one can decompose $A=\frac{\operatorname{Tr}(A)}{n} \mathbb{I}+\widetilde{A}$ where $\operatorname{Tr}(\widetilde{A})=0$. By the definition of primitive Lindblad equation and Lemma II.5, it is equivalent to show that the commutant of $\left\{V_{j}\right\}_{j=1}^{J}$ is spanned by $\mathbb{I}$, that is, if $A$ is in the commutant of $\left\{V_{j}\right\}_{j=1}^{\lrcorner}$, then $\widetilde{A}=0$.

If $A$ is in the commutant, then $\left[\widetilde{A}, V_{j}\right]=0$ for all $j$. Since $\left\{V_{j}\right\}_{j=1}^{J}$ spans the space of traceless matrices, $0=[\widetilde{A},|j\rangle\langle k|]$ for all $j \neq k$, that is, $\widetilde{A}|j\rangle\langle k|=| j\rangle\langle k| \widetilde{A}$. Considering the matrix element $\langle a|\cdot| b\rangle$, we have $\widetilde{A}_{a, j} \delta_{k, b}=\widetilde{A}_{k, b} \delta_{a, j}$. Case (1): take $a=b=k$, then we know that $\widetilde{A}_{k, j}=0$, hence off-diagonal terms of $\widetilde{A}$ are all zero. Case (2): take $a=j$ and $b=k$, then we know that $\widetilde{A}_{j, j}=\widetilde{A}_{k, k} ;$ since $0=\operatorname{Tr}(\widetilde{A})=\sum_{j} \widetilde{A}_{j, j}$, then $\widetilde{A}_{j, j}=0$ for all $j$. In summary, $\widetilde{A}=0$, thus completes the proof. 


\section{Chain rule identity}

One of the key steps in connecting the Lindblad equation and the gradient flow dynamics of the quantum relative entropy is the following chain rule identity. In Sec. III below, this chain rule identity is also the key in proving Lemma III.1.

Lemma II.7 (Chain rule identity [18, Lemma 5.5]). For any $V \in \mathfrak{M}, X \in \mathfrak{P}_{+}$and $\omega \in \mathbb{R}$,

$$
[X]_{\omega}\left(V \log \left(e^{-\omega / 2} X\right)-\log \left(e^{\omega / 2} X\right) V\right)=e^{-\omega / 2} V X-e^{\omega / 2} X V
$$

where the operator $[X]_{\omega}: \mathfrak{M} \rightarrow \mathfrak{M}$ is defined by

$$
[X]_{\omega}(A):=\int_{0}^{1} e^{\omega(s-1 / 2)} X^{s} A X^{1-s} \mathrm{~d} s .
$$

This operator $[X]_{\omega}$ is a noncommutative multiplication of the operator $X$; for convenience, when $\omega=0$, let $[X] \equiv[X]_{0}$. The operator $[X]_{\omega}$ can be extended and defined for vector fields $\mathfrak{M}^{\jmath}$. For $\vec{A}=\left(\begin{array}{llll}A_{1} & A_{2} & \cdots & A_{\lrcorner}\end{array}\right) \in \mathfrak{M}^{\jmath}$ and $\vec{\omega}=\left(\begin{array}{llll}\omega_{1} & \omega_{2} & \cdots & \omega_{\lrcorner}\end{array}\right) \in \mathbb{R}^{\lrcorner}$, define the operator $[X]_{\vec{\omega}}: \mathfrak{M}^{\jmath} \rightarrow \mathfrak{M}^{\jmath}$ by

$$
[X]_{\vec{\omega}}(\vec{A}):=\left([X]_{\omega_{1}}\left(A_{1}\right)[X]_{\omega_{2}}\left(A_{2}\right) \cdots[X]_{\omega_{\jmath}}\left(A_{\jmath}\right)\right) .
$$

As a reminder, these $\omega_{j}$ with $1 \leq j \leq \mathrm{J}$ come from the spectrum of the modular operator $\Delta_{\sigma}$ (see Theorem II.1).

Lemma II.8. If $X \in \mathfrak{P}_{+}$, then for any $\omega \in \mathbb{R},[X]_{\omega}$ is a strictly positive operator on $\mathfrak{M}$. As a consequence, $[X]_{\omega}^{-1}$ is a well-defined strictly positive operator.

Proof. Notice that

$$
\begin{aligned}
\left\langle A,[X]_{\omega} A\right\rangle & =\int_{0}^{1} e^{\omega(s-1 / 2)} \operatorname{Tr}\left(A^{*} X^{s} A X^{1-s}\right) \mathrm{d} s \\
& =\int_{0}^{1} e^{\omega(s-1 / 2)}\left\langle X^{s / 2} A X^{(1-s) / 2}, X^{s / 2} A X^{(1-s) / 2}\right\rangle \mathrm{d} s \geq 0 .
\end{aligned}
$$

Moreover, $\left\langle A,[X]_{\omega} A\right\rangle=0$ iff $A=0$. Thus $[X]_{\omega}$ is a strictly positive operator on $\mathfrak{M}$ for any $\omega \in \mathbb{R}$. This completes the proof.

More explicitly, the inverse of $[X]_{\omega}$ is (see $[18$, Lemma 5.8])

$$
\begin{aligned}
{[X]_{\omega}^{-1}(A) } & =\int_{0}^{\infty} \frac{1}{t+e^{\omega / 2} X} A \frac{1}{t+e^{-\omega / 2} X} \mathrm{~d} t \\
& \equiv \int_{0}^{1} \frac{1}{1-s+s e^{\omega / 2} X} A \frac{1}{1-s+s e^{-\omega / 2} X} \mathrm{~d} s
\end{aligned}
$$

where we change the variable $s=\frac{1}{t+1}$ from the first to the second line above.

By expressions (21) and (23), it is straightforward to verify the following Lemma.

Lemma II.9 ([18, Lemma 5.8]). For any $\omega \in \mathbb{R}$ and $A \in \mathfrak{M}$,

$$
\left([X]_{\omega}(A)\right)^{*}=[X]_{-\omega}\left(A^{*}\right), \quad\left([X]_{\omega}^{-1}(A)\right)^{*}=[X]_{-\omega}^{-1}\left(A^{*}\right) .
$$




\section{E. Noncommutative $\mathbb{L}_{\alpha}$ spaces}

In this subsection, we briefly recall some concepts from noncommutative $\mathbb{L}_{\alpha}$ spaces and logSobolev constants in that setting [44-46], We also recall an implication of the quantum StroockVaropoulos inequality [46], which compares the magnitudes of various log-Sobolev constants.

Let us first introduce the weighted $\mathbb{L}_{\alpha}$ norm, defined as

$$
\|A\|_{\alpha, \sigma}:=\left(\operatorname{Tr}\left(\left|\Gamma_{\sigma}^{1 / \alpha}(A)\right|^{\alpha}\right)\right)^{1 / \alpha} .
$$

Then we need to introduce the power operator, for any $\alpha, \beta \in \mathbb{R}, A \in \mathfrak{M}$,

$$
I_{\beta, \alpha}(A):=\Gamma_{\sigma}^{-\frac{1}{\beta}}\left(\left|\Gamma_{\sigma}^{\frac{1}{\alpha}}(A)\right|^{\frac{\alpha}{\beta}}\right) .
$$

When $A$ is positive and commutes with $\sigma, I_{\beta, \alpha}(A)=A^{\alpha / \beta}$.

Two most important ingredients in noncommutative $\mathbb{L}_{\alpha}$ spaces are entropy function and Dirichlet form. The $\alpha$-Entropy function for $X \in \mathfrak{P}_{+}$is defined as

$$
\begin{aligned}
\operatorname{Ent}_{\alpha, \sigma}(X):= & \operatorname{Tr}\left[\left(\Gamma_{\sigma}^{1 / \alpha}(X)\right)^{\alpha} \log \left(\left(\Gamma_{\sigma}^{1 / \alpha}(X)\right)^{\alpha}\right)\right] \\
& -\operatorname{Tr}\left[\left(\Gamma_{\sigma}^{1 / \alpha}(X)\right)^{\alpha} \log (\sigma)\right]-\|X\|_{\alpha, \sigma}^{\alpha} \log \left(\|X\|_{\alpha, \sigma}^{\alpha}\right),
\end{aligned}
$$

and the $\alpha$-Dirichlet form, for $\alpha \in(0,1) \cup(1, \infty)$, is defined as

$$
\mathcal{E}_{\alpha, \mathcal{L}}(X):=\frac{\alpha \widetilde{\alpha}}{4}\left\langle I_{\widetilde{\alpha}, \alpha}(X),-\mathcal{L}(X)\right\rangle_{1 / 2},
$$

where $1 / \widetilde{\alpha}+1 / \alpha=1$. When $\alpha=1$, the Dirichlet form is defined by taking the limit $\alpha \rightarrow 1$, i.e.,

$$
\mathcal{E}_{1, \mathcal{L}}(X):=\lim _{\alpha \rightarrow 1} \mathcal{E}_{\alpha, \mathcal{L}}(X)=\frac{1}{4}\left\langle\log \left(\Gamma_{\sigma}(X)\right)-\log (\sigma),-\mathcal{L}(X)\right\rangle_{1 / 2} .
$$

The $\alpha$-log Sobolev constant in this setting is defined as

$$
\kappa_{\alpha} \equiv \kappa_{\alpha}(\mathcal{L}):=\inf _{X>0} \frac{\mathcal{E}_{\alpha, \mathcal{L}}(X)}{\operatorname{Ent}_{\alpha, \sigma}(X)},
$$

and thus the quantum $\alpha$-LSI refers to

$$
\kappa_{\alpha} \operatorname{Ent}_{\alpha, \sigma}(X) \leq \mathcal{E}_{\alpha, \mathcal{L}}(X), \quad \forall X>0
$$

In order to compare (5) and (29), for any matrix $X>0$, let us introduce $\rho:=\Gamma_{\sigma}(X) / \operatorname{Tr}\left(\Gamma_{\sigma}(X)\right)$, which is a strictly positive density matrix. By inverting the operator $\Gamma_{\sigma}, X=\operatorname{Tr}\left(\Gamma_{\sigma}(X)\right) \Gamma_{\sigma}^{-1}(\rho)$. By the fact that both $\mathcal{E}_{\alpha, \mathcal{L}}(X)$ and $\operatorname{Ent}_{\alpha, \sigma}(X)$ are homogeneous with respect to $X$ (see, e.g., Proposition 4 (ii) and Proposition 8 (ii) in [46]), we have

$$
\begin{aligned}
\kappa_{\alpha} & =\inf _{\rho \in \mathfrak{D}_{+}} \frac{\mathcal{E}_{\alpha, \mathcal{L}}\left(\Gamma_{\sigma}^{-1}(\rho)\right)}{\operatorname{Ent}_{\alpha, \sigma}\left(\Gamma_{\sigma}^{-1}(\rho)\right)} \\
& =\inf _{\rho \in \mathfrak{D}_{+}} \frac{\frac{\alpha^{2}}{4(\alpha-1)}\left\langle\Gamma_{\sigma^{\alpha}}^{\frac{1-\alpha}{\alpha}}\left(\left(\Gamma_{\sigma^{\frac{1-\alpha}{\alpha}}}(\rho)\right)^{\alpha-1}\right),-\mathcal{L}\left(\Gamma_{\sigma}^{-1}(\rho)\right)\right\rangle_{1 / 2}}{Z D\left(\left(\Gamma_{\sigma}^{(1-\alpha) / \alpha}(\rho)\right)^{\alpha} / Z \| \sigma\right)},
\end{aligned}
$$


where $Z=\operatorname{Tr}\left[\left(\Gamma_{\sigma}^{(1-\alpha) / \alpha}(\rho)\right)^{\alpha}\right]$, which turns out to be the exponent in the sandwiched Rényi divergence (3). Provided that Lindblad equations satisfy KMS-detailed balance, by (12) and Lemma III.1 (see below),

$$
\begin{aligned}
\kappa_{\alpha} & =\inf _{\rho \in \mathfrak{D}_{+}} \frac{\frac{\alpha^{2}}{4(\alpha-1)} \frac{1}{Z}\left\langle\Gamma_{\sigma^{\frac{1-\alpha}{\alpha}}}\left(\left(\Gamma_{\sigma^{\frac{1-\alpha}{\alpha}}}(\rho)\right)^{\alpha-1}\right),-\mathcal{L}^{\dagger}(\rho)\right\rangle}{D\left(\left(\Gamma_{\sigma}^{(1-\alpha) / \alpha}(\rho)\right)^{\alpha} / Z \| \sigma\right)} \\
& =\inf _{\rho \in \mathfrak{D}_{+}} \frac{\frac{\alpha}{4}\left\langle\frac{\delta D_{\alpha}(\rho \| \sigma)}{\delta \rho},-\mathcal{L}^{\dagger}(\rho)\right\rangle}{D\left(\left(\Gamma_{\sigma}^{(1-\alpha) / \alpha}(\rho)\right)^{\alpha} / Z \| \sigma\right)}=\inf _{\rho \in \mathfrak{D}_{+}} \frac{\frac{\alpha}{4} \mathscr{I}_{\alpha}(\rho \| \sigma)}{D\left(\left(\Gamma_{\sigma}^{(1-\alpha) / \alpha}(\rho)\right)^{\alpha} / Z \| \sigma\right)} .
\end{aligned}
$$

As one might observe, the $\alpha$-Dirichlet form becomes the quantum relative $\alpha$-Fisher information up to a multiplicative constant, after the above transformation. However, the denominator in the above equation is different from the sandwiched Rényi divergence, which makes two definitions of the quantum $\alpha$-LSI in (5) and (29) different. When $\alpha=1$, this log-Sobolev constant $\kappa_{1}$ becomes

$$
\kappa_{1}=\frac{1}{4} \inf _{\rho \in \mathfrak{D}_{+}} \frac{\mathscr{I}(\rho \| \sigma)}{D(\rho \| \sigma)}=\frac{1}{4} 2 K=\frac{K}{2} .
$$

Therefore, both definitions (5) and (29) coincide when $\alpha=1$, up to a multiplicative constant $1 / 2$.

The following implication of the quantum Stroock-Varopoulos inequality (see [46, Theorem 14]) characterizes the relationship between various $\kappa_{\alpha}$.

Theorem II.10 ([46, Corollary 16]). Consider primitive Lindblad equations with GNS-detailed balance. Then $\kappa_{\alpha}$ is non-increasing with respect to $\alpha \in[0,2]$. In particular,

$$
\kappa_{1} \geq \kappa_{2}
$$

\section{LINDBLAD EQUATIONS AS THE GRADIENT FLOW DYNAMICS OF THE SANDWICHED RÉNYI DIVERGENCE}

This section devotes to the proof of Theorem 2. We will focus on the case that $\alpha \neq 1$ since the case $\alpha=1$ has been treated previously in [18]. Alternatively, one may easily adapt the proof below to the case $\alpha=1$. The proof follows from a sequence of lemmas whose proofs are postponed to the end of this section.

\section{A. Proof of Theorem I.2}

To simplify notations, for any density matrix $\rho \in \mathfrak{D}$, define

$$
\rho_{\sigma}:=\Gamma_{\sigma}^{\frac{1-\alpha}{\alpha}}(\rho) \equiv \sigma^{\frac{1-\alpha}{2 \alpha}} \rho \sigma^{\frac{1-\alpha}{2 \alpha}} .
$$

Then the sandwiched Rényi divergence for $\alpha \neq 1$ can then be rewritten as

$$
D_{\alpha}(\rho \| \sigma)=\frac{1}{\alpha-1} \log \left(\operatorname{Tr}\left(\rho_{\sigma}^{\alpha}\right)\right) .
$$

The following lemma, connecting the functional derivative of sandwiched Rényi divergence and Lindblad equation, plays an essential role in the proof of Theorem I.2. 
Lemma III.1 (Functional derivative). The functional derivative of the sandwiched Rényi divergence in the space $\mathfrak{D}$ is

$$
\frac{\delta D_{\alpha}(\rho \| \sigma)}{\delta \rho}=\frac{\alpha}{\alpha-1} \frac{\Gamma_{\sigma}^{\frac{1-\alpha}{\alpha}}\left(\rho_{\sigma}^{\alpha-1}\right)}{\operatorname{Tr}\left(\rho_{\sigma}^{\alpha}\right)}
$$

and moreover for $\rho \in \mathfrak{D}_{+}$,

$$
[\rho]_{\alpha, \omega_{j}} \partial_{j}\left(\frac{\delta D_{\alpha}(\rho \| \sigma)}{\delta \rho}\right)=e^{-\omega_{j} / 2} V_{j} \rho-e^{\omega_{j} / 2} \rho V_{j},
$$

where the operator $[X]_{\alpha, \omega}: \mathfrak{M} \rightarrow \mathfrak{M}$, for positive $X \in \mathfrak{P}_{+}$and $\omega \in \mathbb{R}$, is defined by

$$
[X]_{\alpha, \omega}:=\frac{\operatorname{Tr}\left(\left(\Gamma_{\sigma^{\frac{1-\alpha}{\alpha}}}(X)\right)^{\alpha}\right)}{\alpha} \Gamma_{\sigma^{\alpha}}^{\frac{\alpha-1}{\alpha}} \circ\left[\Gamma_{\sigma^{\frac{1-\alpha}{\alpha}}}(X)\right]_{\omega / \alpha} \circ\left[\left(\Gamma_{\sigma^{\alpha}}^{\frac{1-\alpha}{\alpha}}(X)\right)^{\alpha-1}\right]_{(\alpha-1) \omega / \alpha}^{-1} \circ \Gamma_{\sigma^{\alpha}}^{\frac{\alpha-1}{\alpha}} .
$$

Remark III.2. 1) When $\alpha=1, \Gamma_{\sigma}^{\frac{1-\alpha}{\alpha}}=\mathcal{I}$ and $\rho_{\sigma}=\rho$. Then $[\rho]_{\alpha, \omega}$ reduces to $[\rho]_{\omega}$. Hence, $[\rho]_{\alpha, \omega}$ is the generalization of $[\rho]_{\omega}$ to the case of sandwiched Rényi divergence.

2) When $\alpha=2$, the above operator $[\rho]_{2, \omega}$ has a simple form

$$
[\rho]_{2, \omega}=\frac{\operatorname{Tr}\left(\rho_{\sigma}^{2}\right)}{2} \Gamma_{\sigma}=\frac{\operatorname{Tr}\left(\sigma^{-1 / 2} \rho \sigma^{-1 / 2} \rho\right)}{2} \Gamma_{\sigma} .
$$

Thus the mapping

$$
(A, \rho) \rightarrow\left\langle A,[\rho]_{2, \omega}(A)\right\rangle=\frac{\left\langle\rho, \sigma^{-1 / 2} \rho \sigma^{-1 / 2}\right\rangle\left\langle A, \sigma^{1 / 2} A \sigma^{1 / 2}\right\rangle}{2}
$$

is the multiplication of quadratic terms with respect to both $A$ and $\rho$.

The properties of the operator $[X]_{\omega}$ stated in Lemma II.8 and Lemma II.9 generalize to the operator $[X]_{\alpha, \omega}$, as we summarize in the next lemma.

Lemma III.3. If $X \in \mathfrak{P}_{+}$and $\omega \in \mathbb{R}$, then

1. $[X]_{\alpha, \omega}: \mathfrak{M} \rightarrow \mathfrak{M}$ is strictly positive;

2. For any matrix $A$,

$$
\left([X]_{\alpha, \omega}(A)\right)^{*}=[X]_{\alpha,-\omega}\left(A^{*}\right)
$$

The operator $[X]_{\alpha, \omega}$ can be similarly extended to the space $\mathfrak{M}^{\jmath}$. For vector fields $\vec{A}=$ $\left(\begin{array}{llll}A_{1} & A_{2} & \cdots & A_{\lrcorner}\end{array}\right) \in \mathfrak{M}^{\jmath}$ and $\vec{\omega}=\left(\begin{array}{llll}\omega_{1} & \omega_{2} & \cdots & \omega_{\jmath}\end{array}\right) \in \mathbb{R}^{\jmath}$, we define

$$
[X]_{\alpha, \vec{\omega}}(\vec{A}):=\left([X]_{\alpha, \omega_{1}}\left(A_{1}\right)[X]_{\alpha, \omega_{2}}\left(A_{2}\right) \cdots[X]_{\alpha, \omega_{\jmath}}\left(A_{\jmath}\right)\right),
$$

and analogously,

$$
[X]_{\alpha, \vec{\omega}}^{-1}(\vec{A}):=\left([X]_{\alpha, \omega_{1}}^{-1}\left(A_{1}\right)[X]_{\alpha, \omega_{2}}^{-1}\left(A_{2}\right) \cdots \quad[X]_{\alpha, \omega_{\jmath}}^{-1}\left(A_{\jmath}\right)\right) .
$$

The following lemma will be useful to characterize the tangent space of density matrices. 
Lemma III.4. Assume that the Lindblad equation (1) is primitive and $X \in \mathfrak{P}_{+}$. Denote the space of traceless matrices by $\mathfrak{M}_{0}$ and denote the space of traceless Hermitian matrices by $\mathfrak{A}_{0}$, that is, $\mathfrak{A}_{0}=\mathfrak{M}_{0} \cap \mathfrak{A}$.

1. Given $\vec{\omega} \in \mathbb{R}^{J}$, define the operator $\mathfrak{D}_{\alpha, X, \vec{\omega}}: \mathfrak{M}_{0} \rightarrow \mathfrak{M}_{0}$ by

$$
\mathfrak{D}_{\alpha, X, \vec{\omega}}(A):=-\operatorname{div}\left([X]_{\alpha, \vec{\omega}}(\nabla A)\right) .
$$

This operator $\mathfrak{D}_{\alpha, X, \vec{\omega}}$ is strictly positive, and thus invertible.

2. For any $A \in \mathfrak{M}_{0}$,

$$
\left(\mathfrak{D}_{\alpha, X, \vec{\omega}}(A)\right)^{*}=\mathfrak{D}_{\alpha, X, \vec{\omega}}\left(A^{*}\right),
$$

and the restriction of $\mathfrak{D}_{\alpha, X, \vec{\omega}}$ on $\mathfrak{A}_{0}$ is still strictly positive and invertible.

In the case $\alpha=1$, this lemma has been proved in [51, Lemma 3].

Before defining the metric tensor, we need to first introduce an inner product, weighted by $[\rho]_{\alpha, \vec{\omega}}$.

Definition III.5 (Inner product). Given $\alpha \in(0, \infty), \rho \in \mathfrak{D}_{+}$, for vector fields $\vec{A}=\left(\begin{array}{llll}A_{1} & A_{2} & \cdots & A_{\jmath}\end{array}\right)$ and $\vec{B}=\left(\begin{array}{llll}B_{1} & B_{2} & \cdots & B_{\mathrm{J}}\end{array}\right)$, an inner product on vector fields is defined as

$$
\langle\vec{A}, \vec{B}\rangle_{\alpha, \rho, \mathcal{L}}:=\sum_{j=1}^{J}\left\langle A_{j},[\rho]_{\alpha, \omega_{j}}\left(B_{j}\right)\right\rangle .
$$

Given $\rho \in \mathfrak{D}_{+}$, the tangent space is $\mathfrak{A}_{0}$. For $\nu_{1}$ and $\nu_{2} \in \mathfrak{A}_{0}$, by Lemma III.4 part (2), there is a unique $U_{k} \in \mathfrak{A}_{0}$, such that

$$
\nu_{k}=\mathfrak{D}_{\alpha, \rho, \vec{\omega}}\left(U_{k}\right)=-\operatorname{div}\left([\rho]_{\alpha, \vec{\omega}}\left(\nabla U_{k}\right)\right), \quad k=1,2 .
$$

Finally, we are ready to define the metric tensor we need.

Definition III.6 (Metric tensor). Given $\rho \in \mathfrak{D}_{+}$, for $\nu_{k} \in \mathcal{T}_{\rho} \mathfrak{D} \equiv \mathfrak{A}_{0}$, define a metric tensor $g_{\alpha, \rho, \mathcal{L}}$ by

$$
g_{\alpha, \rho, \mathcal{L}}\left(\nu_{1}, \nu_{2}\right):=\left\langle\nabla U_{1}, \nabla U_{2}\right\rangle_{\alpha, \rho, \mathcal{L}} \equiv \sum_{j=1}^{\mathcal{J}}\left\langle\partial_{j} U_{1},[\rho]_{\alpha, \omega_{j}}\left(\partial_{j} U_{2}\right)\right\rangle,
$$

where $U_{k}$ and $\nu_{k}$ are linked via (42).

Proof of Theorem I.2. Up to now, the Riemannian structure $\left(\mathfrak{D}_{+}, g_{\alpha, \rho, \mathcal{L}}\right)$ has been specified, as well as the energy functional $E(\rho) \equiv D_{\alpha}(\rho \| \sigma)$. Then, we will verify that the gradient flow dynamics defined in (10) is exactly the Lindblad equation in (1). To achieve that, we need to compute the gradient at any $\rho_{0} \in \mathfrak{D}_{+}$.

Recall the notion of gradient in the Riemannian manifold (9), $\left.\operatorname{grad} E\right|_{\rho_{0}}$ is defined as the traceless Hermitian matrix such that for any differentiable trajectory $\rho_{t} \in \mathfrak{D}_{+}$with $\rho_{0}$ at time $t=0$,

$$
\left.\left.g_{\alpha, \rho_{0}, \mathcal{L}}\left(\left.\operatorname{grad} E\right|_{\rho_{0}},\left.\dot{\rho}_{t}\right|_{t=0}\right) \equiv \frac{\mathrm{d}}{\mathrm{d} t}\right|_{t=0} E\left(\rho_{t}\right) \equiv \frac{\mathrm{d}}{\mathrm{d} t}\right|_{t=0} D_{\alpha}\left(\rho_{t} \| \sigma\right) .
$$


By direct computation,

$$
\begin{aligned}
\left.\frac{\mathrm{d}}{\mathrm{d} t}\right|_{t=0} D_{\alpha}\left(\rho_{t} \| \sigma\right) & =\left\langle\left.\frac{\delta D_{\alpha}(\rho \| \sigma)}{\delta \rho}\right|_{\rho=\rho_{0}},\left.\dot{\rho}_{t}\right|_{t=0}\right\rangle \\
& =\left\langle\left.\frac{\delta D_{\alpha}(\rho \| \sigma)}{\delta \rho}\right|_{\rho=\rho_{0}},-\operatorname{div}\left(\left[\rho_{0}\right]_{\alpha, \vec{\omega}}\left(\nabla U_{0}\right)\right)\right\rangle \\
& =\left\langle\left.\nabla \frac{\delta D_{\alpha}(\rho \| \sigma)}{\delta \rho}\right|_{\rho=\rho_{0}},\left[\rho_{0}\right]_{\alpha, \vec{\omega}}\left(\nabla U_{0}\right)\right\rangle \\
& =\left\langle\left.\nabla \frac{\delta D_{\alpha}(\rho \| \sigma)}{\delta \rho}\right|_{\rho=\rho_{0}}, \nabla U_{0}\right\rangle_{\alpha, \rho_{0}, \mathcal{L}},
\end{aligned}
$$

where we used the following fact that follows from Lemma III.4: there exists a unique family of traceless Hermitian matrices $\left\{U_{t}\right\}_{t \geq 0}$ such that $\dot{\rho}_{t}=-\operatorname{div}\left(\left[\rho_{t}\right]_{\alpha, \vec{\omega}} \nabla U_{t}\right)$. After combing the last two equations, we have

$$
g_{\alpha, \rho_{0}, \mathcal{L}}\left(\left.\operatorname{grad} E\right|_{\rho_{0}},\left.\dot{\rho}_{t}\right|_{t=0}\right)=\left\langle\left.\nabla \frac{\delta D_{\alpha}(\rho \| \sigma)}{\delta \rho}\right|_{\rho=\rho_{0}}, \nabla U_{0}\right\rangle_{\alpha, \rho_{0}, \mathcal{L}} .
$$

By the definition of the metric tensor $g_{\alpha, \rho, \mathcal{L}}(\cdot, \cdot)(43)$,

$$
\begin{aligned}
\left.\operatorname{grad} E\right|_{\rho_{0}} & =\mathfrak{D}_{\alpha, \rho_{0}, \vec{\omega}}\left(\left.\frac{\delta D_{\alpha}(\rho \| \sigma)}{\delta \rho}\right|_{\rho=\rho_{0}}\right) \\
& =-\operatorname{div}\left(\left[\rho_{0}\right]_{\alpha, \vec{\omega}}\left(\left.\nabla \frac{\delta D_{\alpha}(\rho \| \sigma)}{\delta \rho}\right|_{\rho=\rho_{0}}\right)\right) .
\end{aligned}
$$

Consequently, the gradient flow dynamics (10) is

$$
\dot{\rho}_{t}=-\left.\operatorname{grad} E\right|_{\rho_{t}}=\operatorname{div}\left(\left[\rho_{t}\right]_{\alpha, \vec{\omega}}\left(\left.\nabla \frac{\delta D_{\alpha}(\rho|| \sigma)}{\delta \rho}\right|_{\rho=\rho_{t}}\right)\right) .
$$

Let us rewrite the term on the right hand side

$$
\begin{aligned}
& \operatorname{div}\left(\left[\rho_{t}\right]_{\alpha, \vec{\omega}}\left(\left.\nabla \frac{\delta D_{\alpha}(\rho \| \sigma)}{\delta \rho}\right|_{\rho=\rho_{t}}\right)\right) \\
& \stackrel{(18)}{=} \sum_{j=1}^{J}\left[\left[\rho_{t}\right]_{\alpha, \omega_{j}}\left(\left.\partial_{j} \frac{\delta D_{\alpha}(\rho \| \sigma)}{\delta \rho}\right|_{\rho=\rho_{t}}\right), V_{j}^{*}\right] \\
& \stackrel{(35)}{=} \sum_{j=1}^{J}\left[e^{-\omega_{j} / 2} V_{j} \rho_{t}-e^{\omega_{j} / 2} \rho_{t} V_{j}, V_{j}^{*}\right] \\
& \stackrel{(14)}{=} \mathcal{L}^{\dagger}\left(\rho_{t}\right) .
\end{aligned}
$$

Thus the gradient flow dynamics is exactly the Lindblad equation in (1). 
Proof of Corollary I.3. If $\rho_{t}$ is the solution of the Lindblad equation, then $U_{0}=-\left.\frac{\delta D_{\alpha}(\rho \| \sigma)}{\delta \rho}\right|_{\rho=\rho_{0}}$. Hence,

$$
\begin{aligned}
\left.\frac{\mathrm{d}}{\mathrm{d} t}\right|_{t=0} D_{\alpha}\left(\rho_{t} \| \sigma\right) & =\left\langle\left.\nabla \frac{\delta D_{\alpha}(\rho \| \sigma)}{\delta \rho}\right|_{\rho=\rho_{0}}, \nabla U_{0}\right\rangle_{\alpha, \rho_{0}, \mathcal{L}} \\
& =-\left\langle\left.\nabla \frac{\delta D_{\alpha}(\rho \| \sigma)}{\delta \rho}\right|_{\rho=\rho_{0}},\left.\nabla \frac{\delta D_{\alpha}(\rho \| \sigma)}{\delta \rho}\right|_{\rho=\rho_{0}}\right\rangle_{\alpha, \rho_{0}, \mathcal{L}} \leq 0 .
\end{aligned}
$$

\section{B. Proof of Lemmas}

Here we present the proofs of lemmas that we used in the previous subsection.

Proof of Lemma III.1. For any differentiable curve $\rho_{t} \in \mathfrak{D}$ passing through $\rho$ at time $t=0$, that is, $\rho_{0}=\rho$, we have

$$
\begin{aligned}
\left\langle\frac{\delta D_{\alpha}(\rho \| \sigma)}{\delta \rho}, \dot{\rho}_{0}\right\rangle & \left.\equiv \frac{\mathrm{d}}{\mathrm{d} t}\right|_{t=0} D_{\alpha}\left(\rho_{t} \| \sigma\right) \\
& =\frac{\alpha}{\alpha-1} \frac{\operatorname{Tr}\left(\rho_{\sigma}^{\alpha-1} \sigma^{\frac{1-\alpha}{2 \alpha}} \dot{\rho}_{0} \sigma^{\frac{1-\alpha}{2 \alpha}}\right)}{\operatorname{Tr}\left(\rho_{\sigma}^{\alpha}\right)} \\
& =\frac{\alpha}{\alpha-1} \frac{\operatorname{Tr}\left(\sigma^{\frac{1-\alpha}{2 \alpha}} \rho_{\sigma}^{\alpha-1} \sigma^{\frac{1-\alpha}{2 \alpha}} \dot{\rho}_{0}\right)}{\operatorname{Tr}\left(\rho_{\sigma}^{\alpha}\right)} .
\end{aligned}
$$

Then it is straightforward to obtain (34).

Next, we compute the partial derivative of $\frac{\delta D_{\alpha}(\rho \| \sigma)}{\delta \rho}$. In fact,

$$
\begin{aligned}
& \partial_{j}\left(\frac{\delta D_{\alpha}(\rho \| \sigma)}{\delta \rho}\right) \\
= & \frac{\alpha}{(\alpha-1) \operatorname{Tr}\left(\rho_{\sigma}^{\alpha}\right)}\left[V_{j}, \sigma^{\frac{1-\alpha}{2 \alpha}} \rho_{\sigma}^{\alpha-1} \sigma^{\frac{1-\alpha}{2 \alpha}}\right] \\
= & \frac{\alpha}{(\alpha-1) \operatorname{Tr}\left(\rho_{\sigma}^{\alpha}\right)} \sigma^{\frac{1-\alpha}{2 \alpha}}\left(\sigma^{\frac{\alpha-1}{2 \alpha}} V_{j} \sigma^{\frac{1-\alpha}{2 \alpha}} \rho_{\sigma}^{\alpha-1}-\rho_{\sigma}^{\alpha-1} \sigma^{\frac{1-\alpha}{2 \alpha}} V_{j} \sigma^{\frac{\alpha-1}{2 \alpha}}\right) \sigma^{\frac{1-\alpha}{2 \alpha}} \\
= & \frac{\alpha}{(\alpha-1) \operatorname{Tr}\left(\rho_{\sigma}^{\alpha}\right)} \Gamma^{\frac{1-\alpha}{\alpha}}\left(e^{-\frac{\alpha-1}{2 \alpha} \omega_{j}} V_{j} \rho_{\sigma}^{\alpha-1}-e^{\frac{\alpha-1}{2 \alpha} \omega_{j}} \rho_{\sigma}^{\alpha-1} V_{j}\right) \\
\stackrel{(20)}{=} & \frac{\alpha}{(\alpha-1) \operatorname{Tr}\left(\rho_{\sigma}^{\alpha}\right)} \Gamma_{\sigma}^{\frac{1-\alpha}{\alpha}}\left(\left[\rho_{\sigma}^{\alpha-1}\right]_{\frac{\alpha-1}{\alpha} \omega_{j}}\left(V_{j} \log \left(e^{-\frac{\alpha-1}{2 \alpha} \omega_{j}} \rho_{\sigma}^{\alpha-1}\right)-\log \left(e^{\frac{\alpha-1}{2 \alpha} \omega_{j}} \rho_{\sigma}^{\alpha-1}\right) V_{j}\right)\right) \\
= & \frac{\alpha}{\operatorname{Tr}\left(\rho_{\sigma}^{\alpha}\right)}\left(\Gamma_{\sigma}^{\frac{1-\alpha}{\alpha}} \circ\left[\rho_{\sigma}^{\alpha-1}\right]_{\frac{\alpha-1}{\alpha} \omega_{j}}\right)\left(V_{j} \log \left(e^{-\frac{\omega_{j}}{2 \alpha}} \rho_{\sigma}\right)-\log \left(e^{\frac{\omega_{j}}{2 \alpha}} \rho_{\sigma}\right) V_{j}\right) .
\end{aligned}
$$

To get the fifth line, we have used (20) with $X=\rho_{\sigma}^{\alpha-1}$ and $\omega=\frac{\alpha-1}{\alpha} \omega_{j}$ and $V=V_{j}$. Note that since $\rho, \sigma \in \mathfrak{D}_{+}, \rho_{\sigma}, \rho_{\sigma}^{\alpha-1} \in \mathfrak{P}_{+}$. 
Furthermore, applying (20) again with $X=\rho_{\sigma}, \omega=\omega_{j} / \alpha$ and $V=V_{j}$,

$$
\begin{aligned}
& {\left[\rho_{\sigma}\right]_{\omega_{j} / \alpha}\left(V_{j} \log \left(e^{-\omega_{j} /(2 \alpha)} \rho_{\sigma}\right)-\log \left(e^{\omega_{j} /(2 \alpha)} \rho_{\sigma}\right) V_{j}\right) } \\
= & e^{-\omega_{j} /(2 \alpha)} V_{j} \rho_{\sigma}-e^{\omega_{j} /(2 \alpha)} \rho_{\sigma} V_{j} \\
= & e^{-\omega_{j} /(2 \alpha)} \sigma^{\frac{1-\alpha}{2 \alpha}}\left(\sigma^{\frac{\alpha-1}{2 \alpha}} V_{j} \sigma^{\frac{1-\alpha}{2 \alpha}}\right) \rho \sigma^{\frac{1-\alpha}{2 \alpha}}-e^{\omega_{j} /(2 \alpha)} \sigma^{\frac{1-\alpha}{2 \alpha}} \rho\left(\sigma^{\frac{1-\alpha}{2 \alpha}} V_{j} \sigma^{\frac{\alpha-1}{2 \alpha}}\right) \sigma^{\frac{1-\alpha}{2 \alpha}} \\
= & \Gamma^{\frac{1-\alpha}{\alpha}}\left(e^{-\omega_{j} / 2} V_{j} \rho-e^{\omega_{j} / 2} \rho V_{j}\right) .
\end{aligned}
$$

Therefore,

$$
e^{-\omega_{j} / 2} V_{j} \rho-e^{\omega_{j} / 2} \rho V_{j}=\left(\Gamma_{\sigma^{\alpha}}^{\frac{\alpha-1}{\alpha}} \circ\left[\rho_{\sigma}\right]_{\omega_{j} / \alpha}\right)\left(V_{j} \log \left(e^{-\omega_{j} /(2 \alpha)} \rho_{\sigma}\right)-\log \left(e^{\omega_{j} /(2 \alpha)} \rho_{\sigma}\right) V_{j}\right) .
$$

After plugging it back into (45), one could straightforwardly obtain (35) after arranging a few terms and

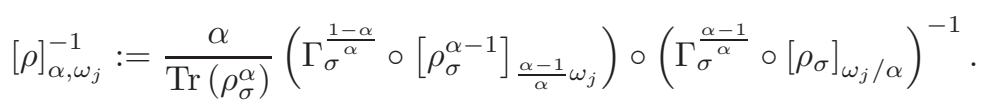

This becomes (36) after we invert this operator.

Proof of Lemma III.3. 1. Since $X \in \mathfrak{P}_{+}, \sigma \in \mathfrak{D}_{+}$, then $X_{\sigma}:=\Gamma_{\sigma}^{\frac{1-\alpha}{\alpha}}(X)$ is strictly positive. For any $A \in \mathfrak{M}$, let $\widetilde{A}:=\Gamma_{\sigma^{\frac{\alpha-1}{\alpha}}}(A)$, then

$$
\begin{aligned}
& \left\langle A,[X]_{\alpha, \omega}(A)\right\rangle \\
= & \frac{\operatorname{Tr}\left(X_{\sigma}^{\alpha}\right)}{\alpha}\left\langle A, \Gamma_{\sigma}^{\frac{\alpha-1}{\alpha}} \circ\left[X_{\sigma}\right]_{\omega / \alpha} \circ\left[X_{\sigma}^{\alpha-1}\right]_{(\alpha-1) \omega / \alpha}^{-1} \circ \Gamma_{\sigma}^{\frac{\alpha-1}{\alpha}}(A)\right\rangle \\
= & \frac{\operatorname{Tr}\left(X_{\sigma}^{\alpha}\right)}{\alpha}\left\langle\widetilde{A},\left[X_{\sigma}\right]_{\omega / \alpha} \circ\left[X_{\sigma}^{\alpha-1}\right]_{(\alpha-1) \omega / \alpha}^{-1}(\widetilde{A})\right\rangle \\
\stackrel{(21)}{=} & \frac{\operatorname{Tr}\left(X_{\sigma}^{\alpha}\right)}{\alpha} \int_{0}^{1} e^{\frac{\omega}{\alpha}\left(s-\frac{1}{2}\right)} \operatorname{Tr}\left(\widetilde{A}^{*} X_{\sigma}^{s}\left(\left[X_{\sigma}^{\alpha-1}\right]_{(\alpha-1) \omega / \alpha}^{-1}(\widetilde{A})\right) X_{\sigma}^{1-s}\right) \mathrm{d} s \\
= & \frac{\operatorname{Tr}\left(X_{\sigma}^{\alpha}\right)}{\alpha} \int_{0}^{1} e^{\frac{\omega}{\alpha}\left(s-\frac{1}{2}\right)} \operatorname{Tr}\left(X_{\sigma}^{\frac{1-s}{2}} \widetilde{A}^{*} X_{\sigma}^{\frac{s}{2}} X_{\sigma}^{\frac{s}{2}}\left(\left[X_{\sigma}^{\alpha-1}\right]_{(\alpha-1) \omega / \alpha}^{-1}(\widetilde{A})\right) X_{\sigma}^{\frac{1-s}{2}}\right) \mathrm{d} s \\
\stackrel{(23)}{=} & \frac{\operatorname{Tr}\left(X_{\sigma}^{\alpha}\right)}{\alpha} \int_{0}^{1} e^{\frac{\omega}{\alpha}\left(s-\frac{1}{2}\right)} \operatorname{Tr}\left(X_{\sigma}^{\frac{1-s}{2}} \widetilde{A}^{*} X_{\sigma}^{\frac{s}{2}}\left(\left[X_{\sigma}^{\alpha-1}\right]_{(\alpha-1) \omega / \alpha}^{-1}\left(X_{\sigma}^{\frac{s}{2}} \widetilde{A} X_{\sigma}^{\frac{1-s}{2}}\right)\right)\right) \mathrm{d} s \\
= & \frac{\operatorname{Tr}\left(X_{\sigma}^{\alpha}\right)}{\alpha} \int_{0}^{1} e^{\frac{\omega}{\alpha}\left(s-\frac{1}{2}\right)}\left\langle X_{\sigma}^{\frac{s}{2}} \widetilde{A} X_{\sigma}^{\frac{1-s}{2}},\left[X_{\sigma}^{\alpha-1}\right]_{(\alpha-1) \omega / \alpha}^{-1}\left(X_{\sigma}^{\frac{s}{2}} \widetilde{A} X_{\sigma}^{\frac{1-s}{2}}\right)\right\rangle \mathrm{d} s \geq 0,
\end{aligned}
$$

since $\left[X_{\sigma}^{\alpha-1}\right]_{(\alpha-1) \omega / \alpha}^{-1}$ is a strictly positive operator by Lemma II.8. Additionally, $\left\langle A,[X]_{\alpha, \omega}(A)\right\rangle=$ 0 if and only if $0=X_{\sigma}^{\frac{s}{2}} \widetilde{A} X_{\sigma^{2}}^{\frac{1-s}{2}} \equiv X_{\sigma}^{\frac{s}{2}}\left(\Gamma_{\sigma^{\frac{\alpha-1}{\alpha}}}(A)\right) X_{\sigma^{\frac{1-s}{2}}}$, which implies that $A=0$. 
2. For any matrix $A$, by Lemma II.9,

$$
\begin{aligned}
\left([X]_{\alpha, \omega}(A)\right)^{*} & =\frac{\operatorname{Tr}\left(X_{\sigma}^{\alpha}\right)}{\alpha} \Gamma_{\sigma^{\alpha-1}}^{\frac{\alpha-1}{\alpha}}\left(\left[X_{\sigma}\right]_{\omega / \alpha} \circ\left[X_{\sigma}^{\alpha-1}\right]_{(\alpha-1) \omega / \alpha}^{-1} \circ \Gamma_{\sigma}^{\frac{\alpha-1}{\alpha}}(A)\right)^{*} \\
& =\frac{\operatorname{Tr}\left(X_{\sigma}^{\alpha}\right)}{\alpha} \Gamma_{\sigma^{\alpha-1}}^{\frac{\alpha-1}{\alpha}} \circ\left[X_{\sigma}\right]_{-\omega / \alpha}\left(\left[X_{\sigma}^{\alpha-1}\right]_{(\alpha-1) \omega / \alpha}^{-1} \circ \Gamma_{\sigma^{\alpha}}^{\alpha-1}(A)\right)^{*} \\
& =\frac{\operatorname{Tr}\left(X_{\sigma}^{\alpha}\right)}{\alpha} \Gamma_{\sigma^{\alpha-1}}^{\frac{\alpha-1}{\alpha}} \circ\left[X_{\sigma}\right]_{-\omega / \alpha} \circ\left[X_{\sigma}^{\alpha-1}\right]_{-(\alpha-1) \omega / \alpha}^{-1}\left(\Gamma_{\sigma}^{\frac{\alpha-1}{\alpha}}(A)\right)^{*} \\
& =\frac{\operatorname{Tr}\left(X_{\sigma}^{\alpha}\right)}{\alpha} \Gamma_{\sigma^{\alpha-1}}^{\frac{\alpha-1}{\alpha}} \circ\left[X_{\sigma}\right]_{-\omega / \alpha} \circ\left[X_{\sigma}^{\alpha-1}\right]_{-(\alpha-1) \omega / \alpha}^{-1} \circ \Gamma_{\sigma}^{\frac{\alpha-1}{\alpha}}\left(A^{*}\right) \\
& =[X]_{\alpha,-\omega}\left(A^{*}\right) .
\end{aligned}
$$

Proof of Lemma III.4. 1. Consider a traceless matrix $A \in \mathfrak{M}_{0}$. It is easy to verify that $\operatorname{Tr}\left(\mathfrak{D}_{\alpha, X, \vec{\omega}}(A)\right)=0$. From the definition of the divergence operator (18), we have

$$
\left\langle A, \mathfrak{D}_{\alpha, X, \vec{\omega}}(A)\right\rangle=\sum_{j=1}^{\mathrm{J}}\left\langle\partial_{j} A,[X]_{\alpha, \omega_{j}}\left(\partial_{j} A\right)\right\rangle \geq 0 .
$$

Moreover, since $[X]_{\alpha, \omega_{j}}$ is strictly positive by Lemma III.3, we know that $\left\langle A, \mathfrak{D}_{\alpha, X, \vec{\omega}}(A)\right\rangle=0$ iff $\partial_{j} A=0$ for all $1 \leq j \leq$ J. Thanks to Lemma II.5 and the assumption that the Lindblad equation (1) is primitive, we have $A=0$ since $A$ is assumed to be traceless. Therefore, $\mathfrak{D}_{\alpha, X, \vec{\omega}}$ is a strictly positive operator on the space of traceless matrices $\mathfrak{M}_{0}$.

2. We only need to prove that $\left(\mathfrak{D}_{\alpha, X, \vec{\omega}}(A)\right)^{*}=\mathfrak{D}_{\alpha, X, \vec{\omega}}\left(A^{*}\right)$. The rest simply follows from the first part of the lemma. Note that

$$
\begin{aligned}
\mathfrak{D}_{\alpha, X, \vec{\omega}}(A) & =-\operatorname{div}\left([X]_{\alpha, \vec{\omega}}(\nabla A)\right) \stackrel{(18)}{=} \sum_{j}\left[V_{j}^{*},[X]_{\alpha, \omega_{j}}\left(\partial_{j} A\right)\right] \\
& =\sum_{j} V_{j}^{*}\left([X]_{\alpha, \omega_{j}}\left(V_{j} A-A V_{j}\right)\right)-\left([X]_{\alpha, \omega_{j}}\left(V_{j} A-A V_{j}\right)\right) V_{j}^{*} .
\end{aligned}
$$

Hence by Theorem II.1 and Lemma III.3, we have

$$
\begin{aligned}
& \left(\mathfrak{D}_{\alpha, X, \vec{\omega}}(A)\right)^{*} \\
= & \sum_{j}\left([X]_{\alpha,-\omega_{j}}\left(A^{*} V_{j}^{*}-V_{j}^{*} A^{*}\right)\right) V_{j}-V_{j}\left([X]_{\alpha,-\omega_{j}}\left(A^{*} V_{j}^{*}-V_{j}^{*} A^{*}\right)\right) \\
= & \sum_{j^{\prime}}\left([X]_{\alpha, \omega_{j^{\prime}}}\left(A^{*} V_{j^{\prime}}-V_{j^{\prime}} A^{*}\right)\right) V_{j^{\prime}}^{*}-V_{j^{\prime}}^{*}\left([X]_{\alpha, \omega_{j^{\prime}}}\left(A^{*} V_{j^{\prime}}-V_{j^{\prime}} A^{*}\right)\right) \\
= & \mathfrak{D}_{\alpha, X, \vec{\omega}}\left(A^{*}\right) .
\end{aligned}
$$




\section{THE NECESSARY CONDITION FOR LINDBLAD EQUATIONS TO BE THE GRADIENT FLOW DYNAMICS OF SANDWICHED RÉNYI DIVERGENCES}

Recall from Theorem I.2 that Lindblad equations with GNS-detailed balance can be regarded as the gradient flow dynamics of sandwiched Rényi divergences, including the quantum relative entropy. A natural and immediate following-up question is the extent that one can possibly generalize Theorem I.2 in the sense of considering a larger family of Lindblad equations. In a recent paper [19], such an issue has been briefly addressed; however, currently, there is still a gap between the class of Lindblad equations (i.e., primitive Lindblad equations with GNS detailed balance) that are known to be the gradient flow dynamics of the quantum relative entropy and the necessary condition (i.e., BKM detailed balance condition) for a primitive Lindblad equation to be possibly expressed as the gradient flow dynamics of the quantum relative entropy; this gap will be revisited and explained in more details below.

In this section, we shall explore the necessary condition for Lindblad equations to be the gradient flow dynamics of sandwiched Rényi divergences, summarized in Theorem IV.1, which adapts the argument from [19, Theorem 2.9]. Next, in Sec. IV B, we will discuss detailed balance conditions arising from Theorem IV.1 and in Sec. IV C, we will discuss relations between various detailed balance conditions.

\section{A. Necessary condition}

Below is the main result of this section. Recall the notation $[X]_{\omega}(21)$ and recall that $[X] \equiv[X]_{0}$.

Theorem IV.1. Suppose that the dual $Q M S \mathcal{P}_{t}^{\dagger}=e^{t \mathcal{L}^{\dagger}}$ is primitive and its unique stationary state is $\sigma \in \mathfrak{D}_{+}$. If there exists a continuously differentiable metric tensor $g_{\rho}(\cdot, \cdot): \mathfrak{A}_{0} \times \mathfrak{A}_{0} \rightarrow \mathbb{R}$ for any

$\rho \in \mathfrak{D}_{+}$such that the Lindblad equation $\dot{\rho}_{t}=\mathcal{L}^{\dagger}\left(\rho_{t}\right)$ is the gradient flow dynamics of the sandwiched Rényi divergence $D_{\alpha}(\rho \| \sigma)$, then $\mathcal{L}$ is self-adjoint with respect to the inner product weighted by an operator $\mathscr{W}_{\sigma, \alpha}: \mathfrak{M} \rightarrow \mathfrak{M}$ defined by

$$
\begin{aligned}
\mathscr{W}_{\sigma, \alpha}(A) & :=\left[\sigma^{\frac{1}{\alpha}}\right] \circ\left[\sigma^{\frac{\alpha-1}{\alpha}}\right]^{-1} \circ \Gamma_{\sigma}^{\frac{2(\alpha-1)}{\alpha}}(A) \\
& \equiv \int_{0}^{1} \int_{0}^{1}\left(\frac{\left(\sigma^{\frac{1}{\alpha}}\right)^{1-s} \sigma^{\frac{\alpha-1}{\alpha}}}{(1-r) \mathbb{I}+r \sigma^{\frac{\alpha-1}{\alpha}}}\right) A\left(\frac{\sigma^{\frac{\alpha-1}{\alpha}}\left(\sigma^{\frac{1}{\alpha}}\right)^{s}}{(1-r) \mathbb{I}+r \sigma^{\frac{\alpha-1}{\alpha}}}\right) \mathrm{d} r \mathrm{~d} s,
\end{aligned}
$$

for all $A \in \mathfrak{M}$; more specifically, we have for all $A, B \in \mathfrak{M}$,

$$
\langle\mathcal{L}(A), B\rangle_{\mathscr{W}_{\sigma, \alpha}}=\langle A, \mathcal{L}(B)\rangle_{\mathscr{W}_{\sigma, \alpha}},
$$

where $\langle A, B\rangle_{\mathscr{W}_{\sigma, \alpha}}:=\left\langle A, \mathscr{W}_{\sigma, \alpha}(B)\right\rangle$.

Remark IV.2. The operator $\mathscr{W}_{\sigma, \alpha}$ is a noncommutative way to multiply $\sigma$ and the condition (49) is equivalent to

$$
\mathscr{W}_{\sigma, \alpha} \circ \mathcal{L} \circ \mathscr{W}_{\sigma, \alpha}^{-1}=\mathcal{L}^{\dagger} .
$$

Motivated by the definition of GNS and KMS detailed balance conditions, we shall define a detailed balance condition based on sandwiched Rényi divergences, for the convenience of discussion below. 
Definition IV.3 (Sandwiched Rényi divergence (SRD) detailed balance condition). For a primitive Lindblad equation with a unique stationary state $\sigma \in \mathfrak{D}_{+}$, it is said to satisfy the sandwiched Rényi divergence $(S R D)$ detailed balance condition if the generator $\mathcal{L}$ is self-adjoint with respect to the inner product $\langle\cdot, \cdot\rangle_{\mathscr{W}_{\sigma, \alpha}}$ for any $\alpha \in(0, \infty)$.

Proof of Theorem IV.1. We first need to introduce some notations following [19, Sec. 2.3]. Define the operator $\mathscr{G}_{\rho}: \mathfrak{A}_{0} \rightarrow \mathfrak{A}_{0}$ by $\left\langle A, \mathscr{G}_{\rho}(B)\right\rangle=g_{\rho}(A, B)$; since $g_{\rho}$ is a $\mathbb{R}$-valued inner product, $\mathscr{G}_{\rho}$ must be invertible and self-adjoint. The inverse of $\mathscr{G}_{\rho}$ is denoted by $\mathscr{K}_{\rho}$, which is also self-adjoint. From (9), one could easily derive that the gradient flow dynamics can be expressed by

$$
\dot{\rho}_{t}=-\mathscr{K}_{\rho_{t}}\left(\left.\frac{\delta D_{\alpha}(\rho \| \sigma)}{\delta \rho}\right|_{\rho=\rho_{t}}\right) .
$$

For convenience, notice that the domain of $\mathscr{K}_{\rho}$ can be extended from $\mathfrak{A}_{0}$ to $\mathfrak{A}$ by setting $\mathscr{K}_{\rho}(\mathbb{I}):=0$.

Under the assumption that $\dot{\rho}_{t}=\mathcal{L}^{\dagger}\left(\rho_{t}\right)$ is the gradient flow dynamics of the sandwiched Rényi divergence $D_{\alpha}(\rho \| \sigma)$ and by $(51)$,

$$
\mathcal{L}^{\dagger}(\rho)=-\mathscr{K}_{\rho}\left(\frac{\delta D_{\alpha}(\rho \| \sigma)}{\delta \rho}\right) .
$$

Choose $\rho_{\epsilon}=\sigma+\epsilon A$ with $A \in \mathfrak{A}_{0}$ and let $\epsilon>0$ small enough such that $\rho_{\epsilon} \in \mathfrak{D}_{+}$. Then

$$
\begin{aligned}
\mathcal{L}^{\dagger}(A) & =\frac{\mathcal{L}^{\dagger}\left(\rho_{\epsilon}\right)-\mathcal{L}^{\dagger}(\sigma)}{\epsilon}=\lim _{\epsilon \downarrow 0^{+}} \frac{\mathrm{d}}{\mathrm{d} \epsilon} \mathcal{L}^{\dagger}\left(\rho_{\epsilon}\right)=-\lim _{\epsilon \downarrow 0^{+}} \frac{\mathrm{d}}{\mathrm{d} \epsilon} \mathscr{K}_{\rho_{\epsilon}}\left(\left.\frac{\delta D_{\alpha}(\rho \| \sigma)}{\delta \rho}\right|_{\rho=\rho_{\epsilon}}\right) \\
& \stackrel{(34)}{=}-\frac{\alpha}{\alpha-1} \lim _{\epsilon \downarrow 0^{+}} \frac{\mathrm{d}}{\mathrm{d} \epsilon}\left(\left(\mathscr{K}_{\sigma}+\epsilon\left(\delta \mathscr{K}_{\sigma}\right)+\mathcal{O}\left(\epsilon^{2}\right)\right)\left(\left.\frac{\Gamma_{\sigma}^{\frac{1-\alpha}{\alpha}}\left(\rho_{\sigma}^{\alpha-1}\right)}{\operatorname{Tr}\left(\rho_{\sigma}^{\alpha}\right)}\right|_{\rho=\rho_{\epsilon}}\right)\right) \\
& =-\frac{\alpha}{\alpha-1} \lim _{\epsilon \downarrow 0^{+}} \frac{\mathrm{d}}{\mathrm{d} \epsilon}\left(\left(\mathscr{K}_{\sigma}+\epsilon\left(\delta \mathscr{K}_{\sigma}\right)+\mathcal{O}\left(\epsilon^{2}\right)\right)\left(\frac{\mathbb{I}+\epsilon B+\mathcal{O}\left(\epsilon^{2}\right)}{1+\mathcal{O}(\epsilon)}\right)\right) \\
& =-\frac{\alpha}{\alpha-1} \mathscr{K}_{\sigma}(B),
\end{aligned}
$$

where the term $B$ in the expansion is (see e.g., [17, Proposition 7.2])

$$
\begin{aligned}
B & =\left.\frac{\mathrm{d}}{\mathrm{d} \epsilon}\right|_{\epsilon=0} \Gamma_{\sigma^{\frac{1-\alpha}{\alpha}}}\left(\left(\Gamma_{\sigma^{\frac{1-\alpha}{\alpha}}}\left(\rho_{\epsilon}\right)\right)^{\alpha-1}\right) \\
& =\Gamma_{\sigma^{\frac{1-\alpha}{\alpha}}}\left(\int_{0}^{1} \int_{0}^{\alpha-1} \frac{\sigma^{\frac{\alpha-1-\beta}{\alpha}}}{(1-s) \mathbb{I}+s \sigma^{\frac{1}{\alpha}}} \Gamma^{\frac{1-\alpha}{\alpha}}(A) \frac{\sigma^{\frac{\beta}{\alpha}}}{(1-s) \mathbb{I}+s \sigma^{\frac{1}{\alpha}}} \mathrm{d} \beta \mathrm{d} s\right) \\
& =(\alpha-1) \int_{0}^{1} \int_{0}^{1} \frac{\left(\sigma^{\frac{\alpha-1}{\alpha}}\right)^{1-\beta}}{(1-s) \mathbb{I}+s \sigma^{\frac{1}{\alpha}}} \Gamma_{\sigma}^{\frac{2(1-\alpha)}{\alpha}}(A) \frac{\left(\sigma^{\frac{\alpha-1}{\alpha}}\right)^{\beta}}{(1-s) \mathbb{I}+s \sigma^{\frac{1}{\alpha}}} \mathrm{d} \beta \mathrm{d} s \\
& =(\alpha-1)\left[\sigma^{\frac{\alpha-1}{\alpha}}\right] \circ\left[\sigma^{\frac{1}{\alpha}}\right]^{-1} \circ \Gamma_{\sigma}^{\frac{2(1-\alpha)}{\alpha}}(A)=(\alpha-1) \mathscr{W}_{\sigma, \alpha}^{-1}(A) .
\end{aligned}
$$

To get the third line, we change the variable $\beta$ by $(\alpha-1) \beta$; in the fourth line, we use expressions $(21)$ and (23). Note that all three operators in the fourth line above pairwise commute. By combining the last two equations,

$$
\mathcal{L}^{\dagger}(A)=-\alpha \mathscr{K}_{\sigma}\left(\mathscr{W}_{\sigma, \alpha}^{-1}(A)\right)
$$


Therefore, for any $C, D \in \mathfrak{A}_{0}$,

$$
\langle\mathcal{L}(C), D\rangle_{\mathscr{W}_{\sigma, \alpha}}=\left\langle C, \mathcal{L}^{\dagger} \circ \mathscr{W}_{\sigma, \alpha}(D)\right\rangle=-\alpha\left\langle C, \mathscr{K}_{\sigma}(D)\right\rangle,
$$

and similarly,

$$
\langle C, \mathcal{L}(D)\rangle_{\mathscr{W}_{\sigma, \alpha}}=(-\alpha)\left\langle\mathscr{K}_{\sigma}(C), D\right\rangle .
$$

As mentioned earlier at the beginning of this proof, the operator $\mathscr{K}_{\sigma}$ is self-adjoint. Thus $\mathcal{L}$ is self-adjoint with respect to the inner product $\langle\cdot, \cdot\rangle_{\mathscr{W}_{\sigma, \alpha}}$.

\section{B. Decomposition of the operator $\mathscr{W}_{\sigma, \alpha}$}

To understand better the operator $\mathscr{W}_{\sigma, \alpha}$, we would like to study its decomposition and various special cases. Suppose the eigenvalue decomposition of $\sigma$ is $\sigma=\sum_{k} \lambda_{k}\left|\psi_{k}\right\rangle\left\langle\psi_{k}\right|$ where $\lambda_{k}>0$ for all $k$. When $\alpha \neq 1$, the expansion of (48) in terms of eigenbasis of $\sigma$ leads into

$$
\mathscr{W}_{\sigma, \alpha}(\cdot)=\sum_{k, j} f_{k, j}^{\alpha}\left|\psi_{k}\right\rangle\left\langle\psi_{k}|\cdot| \psi_{j}\right\rangle\left\langle\psi_{j}\right|
$$

where the coefficients $f_{k, j}^{\alpha}$ are given by

$$
f_{k, j}^{\alpha}=\left\{\begin{aligned}
\lambda_{k}, & \lambda_{k}=\lambda_{j} ; \\
(\alpha-1) \frac{\lambda_{k}^{\frac{1}{\alpha}}-\lambda_{j}^{\frac{1}{\alpha}}}{\lambda_{j}^{\frac{1-\alpha}{\alpha}}-\lambda_{k}^{\frac{1-\alpha}{\alpha}}}, & \lambda_{k} \neq \lambda_{j} .
\end{aligned}\right.
$$

Let us consider a few special weight operators $\mathscr{W}_{\sigma, \alpha}$ :

- $(\alpha=1)$. The operator $\mathscr{W}_{\sigma, \alpha}$ reduces to $[\sigma](\cdot)=\int_{0}^{1} \sigma^{1-s} \cdot \sigma^{s} \mathrm{~d} s$ and the inner product $\langle\cdot, \cdot\rangle_{\mathscr{W}_{\sigma, \alpha}}$ is known as the BKM inner product. As a remark, this case has been shown in [19, Theorem 2.9].

- $(\alpha=2)$. The operator $\mathscr{W}_{\sigma, \alpha}$ reduces to $\Gamma_{\sigma}$ and the inner product $\langle\cdot, \cdot\rangle_{\mathscr{W}_{\sigma, \alpha}}$ is KMS inner product. This immediately implies that in the context of the gradient flow of sandwiched Rényi divergences, one cannot work on a class of Lindblad equations larger than the one with KMS detailed balance condition.

Even though $\alpha \in(0, \infty)$ in the Definition IV.3, we can still apply the limiting argument to define $\mathscr{W}_{\sigma, \infty}$ and $\mathscr{W}_{\sigma, 0}$, so that we could better understand the SRD detailed balance condition.

- $(\alpha=\infty)$. As $\alpha \rightarrow \infty, \sigma^{\frac{1}{\alpha}} \rightarrow \mathbb{I}$ and $\sigma^{\frac{\alpha-1}{\alpha}} \rightarrow \sigma$. As a result, the operator $\mathscr{W}_{\sigma, \alpha}$ converges to $\mathscr{W}_{\sigma, \infty}:=[\sigma]^{-1} \circ \Gamma_{\sigma}^{2}$.

- $(\alpha=0)$. This case is slightly more subtle, since $\sigma^{\frac{\alpha-1}{\alpha}}$ will blow up. When $\lambda_{k} \neq \lambda_{j}$, as $\alpha \downarrow 0$, one could show that $f_{k, j}^{\alpha} \rightarrow \max \left(\lambda_{k}, \lambda_{j}\right)$. Therefore,

$$
\mathscr{W}_{\sigma, 0}(\cdot)=\sum_{k, j} \max \left(\lambda_{k}, \lambda_{j}\right)\left|\psi_{k}\right\rangle\left\langle\psi_{k}|\cdot| \psi_{j}\right\rangle\left\langle\psi_{j}\right| .
$$




\section{Relations between GNS, SRD, KMS detailed balance conditions}

Proposition IV.4. For a primitive Lindblad equation with the unique stationary state $\sigma \in \mathfrak{D}_{+}$,

1. GNS detailed balance condition implies SRD detailed balance condition.

2. SRD detailed balance condition implies KMS detailed balance condition, while conversely it is generally not true.

Remark IV.5. 1. This suggests that the class of Lindblad equations with GNS detailed balance considered in Theorem I.2 seems to be general enough, because it is impossible to generalize Theorem I.2 to the class of Lindblad equations with KMS detailed balance by Proposition IV.4. More specifically, as we shall show later in the proof of Proposition IV.4, there exists at least one primitive Lindblad equation that satisfies KMS detailed balance condition, but does not satisfy SRD detailed balance condition (in particular, $\mathscr{W}_{\sigma, \alpha} \circ \mathcal{L} \circ \mathscr{W}_{\sigma, \alpha}^{-1} \neq \mathcal{L}^{\dagger}$ for any $\alpha \neq 2$ ); because SRD detailed balance condition is necessary for having a gradient flow structure, this particular Lindblad equation cannot be expressed as the gradient flow dynamics of $D_{\alpha}(\rho \| \sigma)$ for any $\alpha \neq 2$.

2. It is still unknown whether the SRD detailed balance condition is equivalent to the GNS detailed balance condition or not. Thus it might be interesting to characterize Lindblad equations with SRD detailed balance, though this task seems to be rather technical.

Proof of Proposition IV.4. The first statement comes immediately by combining Theorem I.2 and Theorem IV.1. As for the second statement, since the SRD detailed balance condition contains KMS detailed balance condition as a special instance when $\alpha=2$, obviously SRD implies KMS. In the following, we provide a primitive two-level Lindblad equation with KMS detailed balance from [18, Appendix B] to demonstrate that KMS does not imply SRD.

Let us consider $K_{1}=|\psi\rangle\langle 0|$ and $K_{2}=|\phi\rangle\langle 1|$ where $|\psi\rangle=\frac{1}{\sqrt{2}}(|0\rangle+|1\rangle)$ and $|\phi\rangle=\frac{1}{\sqrt{5}}(|0\rangle+2|1\rangle)$.

- Define an operator $\mathscr{K}$ by $\mathscr{K}(A):=K_{1}^{*} A K_{1}+K_{2}^{*} A K_{2}$. Thus $\mathscr{K}$ is $\mathrm{CP}$ and $\mathscr{K}\left(\mathbb{I}_{2}\right)=\mathbb{I}_{2}$.

- The adjoint operator

$$
\mathscr{K}^{\dagger}(A)=K_{1} A K_{1}^{*}+K_{2} A K_{2}^{*}=|\psi\rangle\langle 0|A| 0\rangle\langle\psi|+| \phi\rangle\langle 1|A| 1\rangle\langle\phi|
$$

is also CP. Note that $\mathscr{K}^{\dagger}(|0\rangle\langle 1|)=\mathscr{K}^{\dagger}(|1\rangle\langle 0|)=0$ and $\mathscr{K}^{\dagger}(|0\rangle\langle 0|)=|\psi\rangle\langle\psi|$ and $\mathscr{K}^{\dagger}(|1\rangle\langle 1|)=|\phi\rangle\langle\phi|$. It can be readily verified that there is one and only one eigenstate for $\mathscr{K}^{\dagger}$ associated with eigenvalue 1 , which is

$$
\sigma=\frac{1}{7}\left[\begin{array}{ll}
2 & 3 \\
3 & 5
\end{array}\right]
$$

The other non-zero eigenvalue of $\mathscr{K}^{\dagger}$ is $3 / 10$.

- Define $\widetilde{K}_{j}=\sigma^{1 / 2} K_{j}^{*} \sigma^{-1 / 2}$ for $j=1,2$ and define a CP operator $\widetilde{\mathscr{K}}$ via $\widetilde{\mathscr{K}}(A)=\widetilde{K}_{1}^{*} A \widetilde{K}_{1}+$ $\widetilde{K}_{2}^{*} A \widetilde{K}_{2}$. Then $\widetilde{\mathscr{K}}\left(\mathbb{I}_{2}\right)=\mathbb{I}_{2}$ and $\widetilde{\mathscr{K}^{\dagger}}(\sigma)=\sigma$; furthermore, we can verify that

$$
\langle\widetilde{\mathscr{K}} B, A\rangle_{1 / 2}=\langle B, \mathscr{K} A\rangle_{1 / 2} .
$$


Hence, the QMS $\mathcal{P}_{t}=e^{t \mathcal{L}}$ with $\mathcal{L}:=\widetilde{\mathscr{K}} \mathscr{K}-\mathcal{I}_{2}$ can be readily verified to satisfy the KMS detailed balance condition. Moreover, it is easy to show $\mathcal{L}^{\dagger}(\sigma)=0$ and one could verify that it is the only eigenvector of $\mathcal{L}^{\dagger}$ with eigenvalue 0 , i.e., the Lindblad equation is primitive.

Next, we numerically show that (50) does not hold. Figure 1 plots the trace-norm of $\mathscr{W}_{\sigma, \alpha} \circ \mathcal{L} \circ$ $\mathscr{W}_{\sigma, \alpha}^{-1}-\mathcal{L}^{\dagger}$ for various $\alpha$; from this figure, it is clear that (50) holds only when $\alpha=2$ (i.e., KMS detailed balance condition).

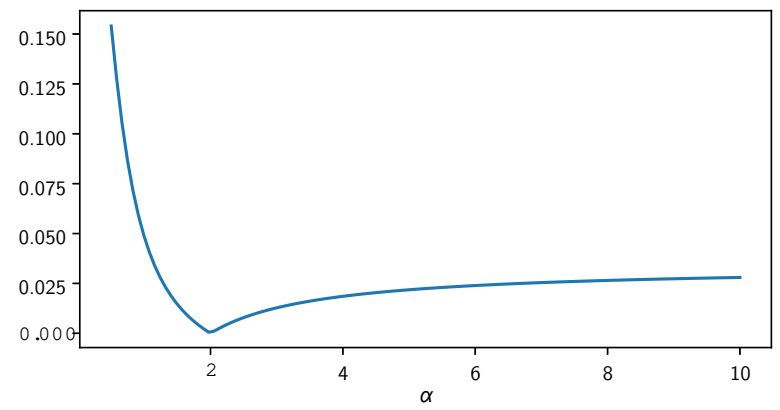

FIG. 1: $\left\|\mathscr{W}_{\sigma, \alpha} \circ \mathcal{L} \circ \mathscr{W}_{\sigma, \alpha}^{-1}-\mathcal{L}^{\dagger}\right\|_{\operatorname{Tr}}$ with respect to various $\alpha$

\section{EXPONENTIAL DECAY OF SANDWICHED RÉNYI DIVERGENCES}

This section is devoted to proving Theorem I.8, i.e., the exponential decay of sandwiched Rényi divergences for primitive Lindblad equations with GNS-detailed balance. We start by recalling the definition of a spectral gap and then we prove a Poincaré inequality (see Proposition V.1). With this Poincaré inequality, we derive a uniform lower bound of the quantum relative 2-Fisher information, which immediately implies Theorem I.8 in the case $\alpha=2$. Next we prove the Proposition I.7, which shows that for primitive Lindblad equations with GNS-detailed balance, the quantum LSI (5) holds for $\alpha=1$ (i.e., there exists $K>0$ ). Then we prove a quantum comparison theorem (see Proposition V.8), which implies that the exponential decay rates of sandwiched Rényi divergences along the Lindblad equation are the same for all $\alpha>1$. This together with the monotonicity of sandwiched Rényi divergences with respect to the order $\alpha$ concludes the proof of Theorem I.8.

\section{A. Spectral gap and Poincaré inequality}

Let us recall from Sec. II that if the Lindblad equation satisfies GNS-detailed balance, then it also satisfies KMS-detailed balance $\left(i . e ., \mathcal{L}\right.$ is self-adjoint with respect to the inner product $\langle\cdot, \cdot\rangle_{1 / 2}$ ). Moreover, by Lemma II.4, $-\mathcal{L}$ is a positive semi-definite operator with respect to the inner product $\langle\cdot, \cdot\rangle_{1 / 2}$. By the additional assumption that the Lindblad equation is primitive, we know $\mathbb{I}$ is the only eigenvector of $-\mathcal{L}$ with respect to the eigenvalue zero. Hence, spectral theory shows that there exists an orthonormal basis $\left\{L_{1}, L_{2}, \cdots, L_{n^{2}-1}, \mathbb{I}\right\}$ such that

$$
-\mathcal{L}\left(L_{j}\right)=\theta_{j} L_{j}, \quad \theta_{1} \geq \theta_{2} \geq \cdots \geq \theta_{n^{2}-1}>0 ; \quad-\mathcal{L}(\mathbb{I})=0 .
$$


The spectral gap of the operator $-\mathcal{L}$ is $\lambda_{\mathcal{L}}:=\theta_{n^{2}-1}$. It is worth remarking that $\left\{L_{1}, L_{2}, \cdots, L_{n^{2}-1}, \mathbb{I}\right\}$ is an orthonormal basis in the Hilbert space $\mathfrak{M}$ equipped with the inner product $\langle\cdot, \cdot\rangle_{1 / 2}$, but not necessarily with other inner products. The following Poincaré inequality follows directly from the definition of the spectral gap.

Proposition V.1 (Poincaré inequality). Assume that the primitive Lindblad equation satisfies $G N S$-detailed balance (1). For any $A \in \mathfrak{M}$ such that $\langle\mathbb{I}, A\rangle_{1 / 2}=0$ (or equivalently, $\left.\operatorname{tr}(\sigma A)=0\right)$,

$$
\langle\nabla A, \nabla A\rangle_{1 / 2}=\langle A,-\mathcal{L}(A)\rangle_{1 / 2} \geq \lambda_{\mathcal{L}}\langle A, A\rangle_{1 / 2}
$$

Moreover, the equality can be achieved when $A=L_{n^{2}-1}$.

Proof. For any $A \in \mathfrak{M}$ such that $\langle\mathbb{I}, A\rangle_{1 / 2}=0$, we know that $A \in \operatorname{span}\left\{L_{j}\right\}_{j=1}^{n^{2}-1}$. Thus by the definition of the spectral gap, $\langle A,-\mathcal{L}(A)\rangle_{1 / 2} \geq \lambda_{\mathcal{L}}\langle A, A\rangle_{1 / 2}$. Moreover, when $A=V_{n^{2}-1}$, the equality holds. Then (57) follows immediately from (19).

Remark V.2. In fact, the above Poincaré inequality is a special case of a whole family of Poincaré inequalities (see [52, Eq. (51)]): given any function $\varphi:(0, \infty) \rightarrow(0, \infty)$, we could consider

$$
\begin{aligned}
C_{\varphi}\left\langle A, \widetilde{\varphi}\left(\Delta_{\sigma}\right)(A)\right\rangle_{1 / 2} & \equiv C_{\varphi}\left\langle A, \varphi\left(\Delta_{\sigma}\right)(A)\right\rangle_{1} \\
& \leq\left\langle A, \varphi\left(\Delta_{\sigma}\right) \circ(-\mathcal{L})(A)\right\rangle_{1} \equiv\left\langle A, \widetilde{\varphi}\left(\Delta_{\sigma}\right) \circ(-\mathcal{L})(A)\right\rangle_{1 / 2}
\end{aligned}
$$

for all $A \in \mathfrak{M}$ such that $\operatorname{Tr}(\sigma A)=0$, where $\widetilde{\varphi}(x):=x^{-1 / 2} \varphi(x)$. The inequality in (57) simply refers to the case $\varphi(x)=x^{1 / 2}$. Because $\left[-\mathcal{L}, \Delta_{\sigma}\right]=0$ (see Lemma II.3), $-\mathcal{L}$ and $\Delta_{\sigma}$ are simultaneously diagonalizable; thus we might as well choose $L_{j}(56)$ in a way that all $L_{j}$ are eigenvectors of both $-\mathcal{L}$ and $\Delta_{\sigma}$. Also note that both $-\mathcal{L}$ and $\Delta_{\sigma}$ are positive semi-definite operators, so they have non-negative spectrum. By expanding $A=\sum_{j=1}^{n^{2}-1} a_{j} L_{j}$, it is not hard to show that $C_{\varphi}=\lambda_{\mathcal{L}}$ in our situation. Therefore, all such Poincaré inequalities have the same prefactor $C_{\varphi}$ for primitive Lindblad equations with GNS-detailed balance.

\section{B. Proof of Theorem I.8 for the case $\alpha=2$}

Proposition V.3 (Lower bound of $\left.\mathscr{I}_{2}(\rho \| \sigma)\right)$. Assume that the primitive Lindblad equation (1) satisfies GNS-detailed balance. Then

$$
\mathscr{I}_{2}(\rho \| \sigma) \geq 2 \lambda_{\mathcal{L}}\left(1-e^{-D_{2}(\rho \| \sigma)}\right), \forall \rho \in \mathfrak{D}
$$

Proof. The case $\alpha=2$ is easier to deal with as certain quantities become more explicit:

$$
\begin{aligned}
\frac{\delta D_{2}(\rho \| \sigma)}{\delta \rho} & =\frac{2 \sigma^{-1 / 2} \rho \sigma^{-1 / 2}}{\left\langle\rho, \sigma^{-1 / 2} \rho \sigma^{-1 / 2}\right\rangle}=\frac{2 \Gamma_{\sigma}^{-1}(\rho)}{\left\langle\Gamma_{\sigma}^{-1}(\rho), \Gamma_{\sigma}^{-1}(\rho)\right\rangle_{1 / 2}} \\
D_{2}(\rho \| \sigma) & =\log \operatorname{Tr}\left(\sigma^{-1 / 2} \rho \sigma^{-1 / 2} \rho\right)=\log \left(\left\langle\Gamma_{\sigma}^{-1}(\rho), \Gamma_{\sigma}^{-1}(\rho)\right\rangle_{1 / 2}\right) .
\end{aligned}
$$


Then, by the definition of the quantum relative $\alpha$-Fisher information (4)

$$
\begin{aligned}
\mathscr{I}_{2}(\rho \| \sigma) & =-\left\langle\frac{2 \Gamma_{\sigma}^{-1}(\rho)}{\left\langle\Gamma_{\sigma}^{-1}(\rho), \Gamma_{\sigma}^{-1}(\rho)\right\rangle_{1 / 2}}, \mathcal{L}^{\dagger}(\rho)\right\rangle \\
& =\frac{-2}{\left\langle\Gamma_{\sigma}^{-1}(\rho), \Gamma_{\sigma}^{-1}(\rho)\right\rangle_{1 / 2}}\left\langle\Gamma_{\sigma}^{-1}(\rho), \Gamma_{\sigma}^{-1} \circ \mathcal{L}^{\dagger} \circ \Gamma_{\sigma}\left(\Gamma_{\sigma}^{-1}(\rho)\right)\right\rangle_{1 / 2} \\
& \stackrel{(12)}{=} \frac{-2}{\left\langle\Gamma_{\sigma}^{-1}(\rho), \Gamma_{\sigma}^{-1}(\rho)\right\rangle_{1 / 2}}\left\langle\Gamma_{\sigma}^{-1}(\rho), \mathcal{L}\left(\Gamma_{\sigma}^{-1}(\rho)\right)\right\rangle_{1 / 2} \\
& =\frac{-2}{\left\langle\Gamma_{\sigma}^{-1}(\rho), \Gamma_{\sigma}^{-1}(\rho)\right\rangle_{1 / 2}}\left\langle\Gamma_{\sigma}^{-1}(\rho-\sigma), \mathcal{L}\left(\Gamma_{\sigma}^{-1}(\rho-\sigma)\right)\right\rangle_{1 / 2} \\
& \stackrel{(57)}{\geq} \frac{2}{\left\langle\Gamma_{\sigma}^{-1}(\rho), \Gamma_{\sigma}^{-1}(\rho)\right\rangle_{1 / 2}} \lambda_{\mathcal{L}}\left\langle\Gamma_{\sigma}^{-1}(\rho-\sigma), \Gamma_{\sigma}^{-1}(\rho-\sigma)\right\rangle_{1 / 2} .
\end{aligned}
$$

It is not hard to verify that

$$
\left\langle\Gamma_{\sigma}^{-1}(\rho), \Gamma_{\sigma}^{-1}(\rho)\right\rangle_{1 / 2}=1+\left\langle\Gamma_{\sigma}^{-1}(\rho-\sigma), \Gamma_{\sigma}^{-1}(\rho-\sigma)\right\rangle_{1 / 2}
$$

It is interesting to note that the term $\left\langle\Gamma_{\sigma}^{-1}(\rho-\sigma), \Gamma_{\sigma}^{-1}(\rho-\sigma)\right\rangle_{1 / 2}$ turns out to be the quantum $\chi^{2}$-divergence $\chi_{1 / 2}^{2}(\rho, \sigma)$ studied in [37]. Then

$$
\begin{aligned}
\mathscr{I}_{2}(\rho \| \sigma) & \geq \frac{2 \lambda_{\mathcal{L}}}{\left\langle\Gamma_{\sigma}^{-1}(\rho), \Gamma_{\sigma}^{-1}(\rho)\right\rangle_{1 / 2}}\left(\left\langle\Gamma_{\sigma}^{-1}(\rho), \Gamma_{\sigma}^{-1}(\rho)\right\rangle_{1 / 2}-1\right) \\
& =\frac{2 \lambda_{\mathcal{L}}}{e^{D_{2}(\rho \| \sigma)}}\left(e^{D_{2}(\rho \| \sigma)}-1\right)
\end{aligned}
$$

which yields (58).

With Proposition V.3, we can immediately show the exponential decay of the sandwiched Rényi divergence of order $\alpha=2$.

Proof of Theorem I.8 for the case $\alpha=2$. From the definition of the quantum relative 2-Fisher information in (4) and by Proposition V.3,

$$
\frac{\mathrm{d}}{\mathrm{d} t} D_{2}\left(\rho_{t} \| \sigma\right)=-\mathscr{I}_{2}\left(\rho_{t} \| \sigma\right) \leq-2 \lambda_{\mathcal{L}}\left(1-e^{-D_{2}\left(\rho_{t} \| \sigma\right)}\right) .
$$

Then $\frac{\mathrm{d}}{\mathrm{d} t} \log \left(e^{D_{2}\left(\rho_{t} \| \sigma\right)}-1\right) \leq-2 \lambda_{\mathcal{L}}$. After integrating it from time 0 to $t$, one could find after some straightforward simplification that

$$
D_{2}\left(\rho_{t} \| \sigma\right) \leq \log \left(1+\left(e^{D_{2}\left(\rho_{0} \| \sigma\right)}-1\right) e^{-2 \lambda_{\mathcal{L}} t}\right) \leq\left(e^{D_{2}\left(\rho_{0} \| \sigma\right)}-1\right) e^{-2 \lambda_{\mathcal{L}} t} .
$$

Thus $D_{2}\left(\rho_{t} \| \sigma\right)$ decays exponentially fast with rate $2 \lambda_{\mathcal{L}}$. Apparently, the prefactor $C_{2, \epsilon}$ and the waiting time $\tau_{2, \epsilon}$ could be chosen as

$$
C_{2, \epsilon}=\frac{1}{D_{2}\left(\rho_{0} \| \sigma\right)}\left(e^{D_{2}\left(\rho_{0} \| \sigma\right)}-1\right), \quad \tau_{2, \epsilon}=0 .
$$


The lower bound of $\mathscr{I}_{2}(\rho \| \sigma)$ in Proposition V.3 immediately leads into a lower bound of the quantum 2-log Sobolev constant $K_{2}$ in (5).

Corollary V.4 ([39, Theorem 4.2]). For primitive Lindblad equations with GNS-detailed balance, the quantum 2-log Sobolev constant $K_{2}$ is bounded below by the spectral gap; specifically,

$$
K_{2} \geq \frac{1-\lambda_{\min }(\sigma)}{\log \left(\lambda_{\min }(\sigma)^{-1}\right)} \lambda_{\mathcal{L}}
$$

Proof. From Proposition V.3, we know that

$$
\frac{\mathscr{I}_{2}(\rho \| \sigma)}{D_{2}(\rho \| \sigma)} \geq 2 \lambda_{\mathcal{L}} \frac{1-e^{-D_{2}(\rho \| \sigma)}}{D_{2}(\rho \| \sigma)} \geq 2 \lambda_{\mathcal{L}} \frac{1-\lambda_{\min }(\sigma)}{\log \left(\lambda_{\min }(\sigma)^{-1}\right)}
$$

because $x \rightarrow \frac{1-e^{-x}}{x}$ is monotonically decreasing on $(0, \infty)$ and $0 \leq D_{2}(\rho \| \sigma) \leq \log \left(\lambda_{\min }(\sigma)^{-1}\right)[39$, Lemma 2.1]. The above result follows immediately by taking the minimization over $\rho$ for both sides.

\section{Proof of the quantum LSI}

In this subsection, we prove the Proposition I.7, which shows the equivalence of the primitivity and the validity of quantum LSI (5), for Lindblad equations with GNS-detailed balance.

One key ingredient is the $l_{2}$ mixing time defined as the following for any $\epsilon>0$,

$$
\begin{aligned}
t_{2}(\epsilon) & =\inf \left\{t \geq 0:\left\|e^{t \mathcal{L}}(A)-\operatorname{Tr}(\sigma A) \mathbb{I}\right\|_{2, \sigma} \leq \epsilon, \forall A \in \mathfrak{M} \text { s.t. }\|A\|_{1, \sigma}=1\right\} \\
& \equiv \inf \left\{t \geq 0:\left\|e^{t \mathcal{L}}(\cdot)-\operatorname{Tr}(\sigma \cdot) \mathbb{I}\right\|_{1 \rightarrow 2, \sigma} \leq \epsilon\right\} .
\end{aligned}
$$

We shall need the following two results.

Lemma V.5. Under the same assumption as in Proposition I.7, if the Lindblad equation is primitive with the spectral gap $\lambda_{\mathcal{L}}$, then

$$
t_{2}(\epsilon) \leq \max \left(0, \frac{1}{2 \lambda_{\mathcal{L}}} \log \left(\frac{1}{\lambda_{\min }(\sigma) \epsilon^{2}}\right)\right) .
$$

Its proof will be given slightly later in this subsection. As a remark, when $\epsilon \geq \sqrt{\frac{1}{\lambda_{\min }(\sigma)}}$, we have $t_{2}(\epsilon)=0$; this is intuitively reasonable since when $\epsilon$ is large enough, the inequality in (61) trivially holds.

Theorem V.6 ([39, Theorem 5.3]). Under the same assumption as in Proposition I.7, if the Lindblad equation is primitive, then

$$
t_{2}\left(e^{-1}\right) \kappa_{2} \geq \frac{1}{2}
$$

Proof of Proposition I.7. First, recall that [45, Theorem 16] has proved that $K \leq \lambda_{\mathcal{L}}$ by linearizing the quantum LSI, thus $K>0$ implies $\lambda_{\mathcal{L}}>0$. 
As for the other direction, assume that the Lindblad equation is primitive. Suppose $\rho_{t}=e^{t \mathcal{L}^{\dagger}}\left(\rho_{0}\right)$ is the solution of the Lindblad equation. Combining the estimates above, we have

$$
K \stackrel{(31)}{=} 2 \kappa_{1} \stackrel{(32)}{\geq} 2 \kappa_{2} \stackrel{(63)}{\geq} \frac{1}{t_{2}\left(e^{-1}\right)} \stackrel{(62)}{\geq} \frac{2 \lambda_{\mathcal{L}}}{2-\log \left(\lambda_{\min }(\sigma)\right)}=\frac{1}{1-\log \left(\sqrt{\lambda_{\min }(\sigma)}\right)} \lambda_{\mathcal{L}}
$$

Remark V.7. In the proof above, we use the mixing time $t_{2}\left(e^{-1}\right)$ as a bridge to connect $\kappa_{2}$ and $\lambda_{\mathcal{L}}$, and use the quantum Stroock-Varopoulos inequality to connect $K$ and $\kappa_{2}$. To the best of our knowledge, a direct proof of the quantum LSI (5) has not appeared in the literature. For classical systems, Bakry-Émery [53] condition is an important criterion for classical LSI to hold for Fokker-Planck equations. It is an interesting open question to see whether there is a quantum analog.

Proof of Lemma V.5. Let us introduce $B=e^{t \mathcal{L}}(A)-\operatorname{Tr}(\sigma A) \mathbb{I}$ and decompose $A=a_{0} \mathbb{I}+\sum_{j=1}^{n^{2}-1} a_{j} L_{j}$, where $L_{j}$ are eigenvectors of the operator $\mathcal{L}$; recall (56) for notations. Then we easily know that $B=\sum_{j=1}^{n^{2}-1} a_{j} e^{-t \theta_{j}} L_{j}$ and that

$$
\begin{aligned}
\left\|e^{t \mathcal{L}}(A)-\operatorname{Tr}(\sigma A) \mathbb{I}\right\|_{2, \sigma}^{2} & =\operatorname{Tr}\left(\sigma^{\frac{1}{4}} B^{*} \sigma^{\frac{1}{4}} \sigma^{\frac{1}{4}} B \sigma^{\frac{1}{4}}\right)=\langle B, B\rangle_{1 / 2} \\
& =\sum_{j=1}^{n^{2}-1} e^{-2 t \theta_{j}}\left|a_{j}\right|^{2} \leq e^{-2 t \lambda_{\mathcal{L}}} \sum_{j=1}^{n^{2}-1}\left|a_{j}\right|^{2} .
\end{aligned}
$$

Let $\widetilde{A}:=\left|\sigma^{\frac{1}{2}} A \sigma^{\frac{1}{2}}\right|$. From the condition that $\|A\|_{1, \sigma}=1$ in $(61)$, we have $\operatorname{Tr}(\widetilde{A})=1$. Then

$$
\begin{aligned}
1 & \geq \operatorname{Tr}\left(\widetilde{A}^{2}\right)=\operatorname{Tr}\left(\sigma^{\frac{1}{2}} A^{*} \sigma^{\frac{1}{2}} \sigma^{\frac{1}{2}} A \sigma^{\frac{1}{2}}\right)=\left\langle\sigma^{\frac{1}{4}}\left(\sigma^{\frac{1}{4}} A \sigma^{\frac{1}{4}}\right) \sigma^{\frac{1}{4}}, \sigma^{\frac{1}{4}}\left(\sigma^{\frac{1}{4}} A \sigma^{\frac{1}{4}}\right) \sigma^{\frac{1}{4}}\right\rangle \\
& \geq \lambda_{\min }(\sigma)\left\langle\sigma^{\frac{1}{4}} A \sigma^{\frac{1}{4}}, \sigma^{\frac{1}{4}} A \sigma^{\frac{1}{4}}\right\rangle=\lambda_{\min }(\sigma)\langle A, A\rangle_{1 / 2} \geq \lambda_{\min }(\sigma)\left(\sum_{j=1}^{n^{2}-1}\left|a_{j}\right|^{2}\right) .
\end{aligned}
$$

Therefore, we know that

$$
\left\|e^{t \mathcal{L}}(A)-\operatorname{Tr}(\sigma A) \mathbb{I}\right\|_{2, \sigma}^{2} \leq e^{-2 t \lambda_{\mathcal{L}}} \frac{1}{\lambda_{\min }(\sigma)} .
$$

Easily, we know that whenever $t \geq \frac{1}{2 \lambda_{\mathcal{L}}} \log \left(\frac{1}{\lambda_{\min }(\sigma) \epsilon^{2}}\right)$, we have $\left\|e^{t \mathcal{L}}(A)-\operatorname{Tr}(\sigma A) \mathbb{I}\right\|_{2, \sigma} \leq \epsilon$. Then (62) follows immediately.

\section{Proof of Theorem I.8 for $\alpha \in(0, \infty)$ via a quantum comparison theorem}

As a quantum analog of [28, Lemma 3.4] for Fokker-Planck equations and [25, Theorem 3.2.3] for the Ornstein-Uhlenbeck process, in Proposition V.8, we will show that under primitive Lindblad equations with GNS-detailed balance (1), the sandwiched Rényi divergence of higher order $\alpha_{1}$ can be bounded above by the sandwiched Rényi divergence of lower order $\alpha_{0}\left(1<\alpha_{0}<\alpha_{1}\right)$, at the expense of some time delay. This result helps to prove the exponential decay for sandwiched Rényi divergences under Lindblad equations, summarized in Proposition V.10. 
Proposition V.8 (Quantum comparison theorem). Let $\rho_{t}$ be the solution of the Lindblad equation with GNS-detailed balance (1). Assume that the quantum LSI (5) holds with constant K. Assume also that

$$
D\left(\rho_{0} \| \sigma\right) \leq \epsilon \text { for some fixed } \epsilon \in\left(0, \frac{\lambda_{\min }(\sigma)^{2}}{2}\right) .
$$

Then for any two orders $1<\alpha_{0} \leq \alpha_{1}$, we have

$$
D_{\alpha_{1}}\left(\rho_{T} \| \sigma\right) \leq D_{\alpha_{0}}\left(\rho_{0} \| \sigma\right)
$$

with

$$
T=\frac{1}{2 K \eta} \log \left(\frac{\alpha_{1}-1}{\alpha_{0}-1}\right)
$$

where

$$
\begin{aligned}
& \eta=\min \left(\frac{1}{2}, \min _{j=1}^{\jmath}\left\{\frac{2 \sqrt{e^{\omega_{j}} \frac{1}{\Lambda}}}{1+e^{\omega_{j}} \Lambda}\right\}\right), \\
& \Lambda=\frac{\lambda_{\max }(\sigma)}{\lambda_{\min }(\sigma)} \exp \left(\alpha_{0} \sqrt{2 \epsilon} \frac{2 \lambda_{\min }(\sigma)-\sqrt{2 \epsilon}}{\lambda_{\min }(\sigma)\left(\lambda_{\min }(\sigma)-\sqrt{2 \epsilon}\right)}\right) .
\end{aligned}
$$

The proof is postponed to Sec. VE. Later in Sec. VF, we will discuss this quantum comparison theorem in details. As a reminder, we have, in fact, implicitly assumed that Lindblad equations under consideration are primitive, by Proposition I.7.

Remark V.9. 1. The assumption (64) on the initial condition $\rho_{0}$ is merely a technical assumption allowing us to obtain a neat expression for the delay time $T$. This assumption (64) is not essential for the inequality (65) to hold; one could remove the assumption at the expense of longer delay time. In fact, the validity of the quantum LSI (5) leads to the exponential decay of the quantum relative entropy, so that $D\left(\rho_{t_{0}} \| \sigma\right) \leq \epsilon$ holds for some $t_{0} \geq 0$. Taking $\rho_{t_{0}}$ in place of $\rho_{0}$, one sees that this would imply (65) with a larger decay time $T$.

2. Another reason for imposing the assumption (64) is to avoid technicalities. Thanks to the quantum Pinsker's inequality

$$
D(\rho \| \sigma) \geq \frac{1}{2}(\operatorname{Tr}|\rho-\sigma|)^{2}
$$

the assumption that $D\left(\rho_{0} \| \sigma\right) \leq \epsilon$ with $0<\epsilon<\frac{\lambda_{\min }(\sigma)^{2}}{2}$ implies that $\rho_{0}$ is a strictly positive density matrix; in fact, we can even show that $\rho_{t} \in \mathfrak{D}_{+}$, for any $t \geq 0$. This assumption can help avoid many technical issues arising from degenerate density matrices and it validates the usage of results in the previous two sections, e.g., properties of the operator $[X]_{\omega}$ (which require $X$ to be strictly positive).

Proposition V.10. Let $\rho_{t}$ be the solution of the Lindblad equation with GNS-detailed balance (1). Assume that the quantum LSI (5) holds with constant $K$. If the sandwiched Rényi divergence decays exponentially fast with rate $R$ for some order $\alpha_{0}>1$, then it decays exponentially fast for any $\alpha \in(0, \infty)$ with rate at least $R$. 
Proof of Proposition V.10. The exponential decay of the sandwiched Rényi divergence means there exists some time $t_{0}$ and constant $C_{0}$ such that

$$
D_{\alpha_{0}}\left(\rho_{t} \| \sigma\right) \leq C_{0} D_{\alpha_{0}}\left(\rho_{0} \| \sigma\right) e^{-R t}, \quad \forall t \geq t_{0} .
$$

Case (I): $\alpha \leq \alpha_{0}$. By the monotonicity of sandwiched Rényi divergences with respect to the order $\alpha[34$, Theorem 7],

$$
D_{\alpha}\left(\rho_{t} \| \sigma\right) \leq D_{\alpha_{0}}\left(\rho_{t} \| \sigma\right) \leq C_{0} D_{\alpha_{0}}\left(\rho_{0} \| \sigma\right) e^{-R t}=C_{\alpha} D_{\alpha}\left(\rho_{0} \| \sigma\right) e^{-R t},
$$

for all $t \geq t_{0}$, where $C_{\alpha}=\frac{C_{0} D_{\alpha_{0}}\left(\rho_{0} \| \sigma\right)}{D_{\alpha}\left(\rho_{0} \| \sigma\right)}$.

Case (II): $\alpha>\alpha_{0}$. Pick an arbitrary $0<\epsilon<\frac{\lambda_{\min }(\sigma)^{2}}{2}$. Notice that the quantum LSI implies the exponential decay of the quantum relative entropy, namely,

$$
D\left(\rho_{t} \| \sigma\right) \leq D\left(\rho_{0} \| \sigma\right) e^{-2 K t} .
$$

Hence when $t \geq \frac{1}{2 K} \log \left(\frac{D\left(\rho_{0} \| \sigma\right)}{\epsilon}\right)$, one has $D\left(\rho_{t} \| \sigma\right) \leq \epsilon$. Moreover, if $t \geq T+\max \left(t_{0}, \frac{1}{2 K} \log \left(\frac{D\left(\rho_{0} \| \sigma\right)}{\epsilon}\right)\right)$, where $T$ is the time delay given in Proposition V.8 with the choice $\alpha_{1}=\alpha$, then one obtains from Proposition V.8 that

$$
D_{\alpha}\left(\rho_{t} \| \sigma\right) \leq D_{\alpha_{0}}\left(\rho_{t-T} \| \sigma\right) \leq C_{0} D_{\alpha_{0}}\left(\rho_{0} \| \sigma\right) e^{-R(t-T)}=C_{\alpha} D_{\alpha}\left(\rho_{0} \| \sigma\right) e^{-R t},
$$

where $C_{\alpha}=\frac{C_{0} D_{\alpha_{0}}\left(\rho_{0} \| \sigma\right) e^{R T}}{D_{\alpha}\left(\rho_{0} \| \sigma\right)}$.

Combining both cases above finishes the proof of Proposition V.10.

Proof of Theorem I.8. Set $\alpha_{0}=2$ and $R=2 \lambda_{\mathcal{L}}$ in Proposition V.10. Theorem I.8 follows immediately from Proposition V.10 and the case $\alpha=2$ proved in Sec. VB; the validity of the quantum LSI has been shown in Proposition I.7. The expression of $T_{\alpha, \epsilon}$ follows immediately from Proposition V.8.

\section{E. Proof of quantum comparison theorem}

We now turn to the proof of Proposition V.8. Consider the density matrix

$$
\varrho=\frac{\left(\Gamma_{\sigma^{\frac{1-\alpha}{\alpha}}}(\rho)\right)^{\alpha}}{Z} \equiv \frac{\rho_{\sigma}^{\alpha}}{Z},
$$

where the normalization constant $Z=\operatorname{Tr}\left(\rho_{\sigma}^{\alpha}\right)$ and $\rho \in \mathfrak{D}_{+}$. Then apparently, $\varrho \in \mathfrak{D}_{+}$. The $\log$-Sobolev inequality (5), with $\rho$ in (5) chosen as $\varrho$ above, becomes

$$
\operatorname{Tr}\left(\rho_{\sigma}^{\alpha} \log \left(\rho_{\sigma}^{\alpha}\right)\right)-Z \log (Z)-\operatorname{Tr}\left(\rho_{\sigma}^{\alpha} \log (\sigma)\right) \leq \frac{1}{2 K} \operatorname{Tr}\left(-\mathcal{L}^{\dagger}\left(\rho_{\sigma}^{\alpha}\right)\left(\log \left(\rho_{\sigma}^{\alpha}\right)-\log (\sigma)\right)\right) .
$$

This inequality can be regarded as a variant of the log-Sobolev inequality and it will be used later. Let us define the operator $G_{X, \omega}(s): \mathfrak{M} \rightarrow \mathfrak{M}$ by

$$
G_{X, \omega}(s)(A):=e^{\omega s} X^{s} A X^{-s}+e^{\omega(1-s)} X^{1-s} A X^{-(1-s)} .
$$

The lemma below collects some properties of $G_{X, \omega}(s)$ which will be useful in the proof of Proposition V.8. 
Lemma V.11. Assume that $X \in \mathfrak{P}_{+}$and $\omega \in \mathbb{R}$. Then the operator $G_{X, \omega}(s)$ satisfies:

1. For $0 \leq s_{1}<s_{2} \leq \frac{1}{2}$,

$$
G_{X, \omega}\left(s_{1}\right) \geq G_{X, \omega}\left(s_{2}\right) .
$$

Moreover, the equality is obtained iff $\omega=0$ and $X=c \mathbb{I}$ for some constant $c>0$.

2. For $s \in\left[0, \frac{1}{2}\right]$, a bound for the spectrum of $G_{X, \omega}(s)$ is

$$
2 \sqrt{e^{\omega} \frac{\lambda_{\min }(X)}{\lambda_{\max }(X)}} \mathcal{I} \leq G_{X, \omega}(s) \leq\left(1+e^{\omega} \frac{\lambda_{\max }(X)}{\lambda_{\min }(X)}\right) \mathcal{I} .
$$

As a consequence,

$$
\eta G_{X, \omega}\left(s_{1}\right) \leq G_{X, \omega}\left(s_{2}\right), \quad \forall s_{1}, s_{2} \in\left[0, \frac{1}{2}\right]
$$

with

$$
\eta=\frac{2 \sqrt{e^{\omega} \frac{\lambda_{\min }(X)}{\lambda_{\max }(X)}}}{1+e^{\omega} \frac{\lambda_{\max }(X)}{\lambda_{\min }(X)}} .
$$

Proof of Lemma V.11. 1. For any positive number $b$, notice that $b^{s}+b^{1-s}$ is a non-increasing convex function with respect to $s$ on the interval $\left[0, \frac{1}{2}\right]$. Moreover,

- the range of the function $b^{s}+b^{1-s}$ on this interval is $[2 \sqrt{b}, 1+b]$;

- $b^{s_{1}}+b^{1-s_{1}}=b^{s_{2}}+b^{1-s_{2}}$ for some $0 \leq s_{1}<s_{2} \leq \frac{1}{2}$ iff $b=1$.

Let us write the spectral decomposition of $X$ as $X=\sum_{k=1}^{n} \lambda_{k}|k\rangle\langle k|$ where $\lambda_{k}>0$ by assumption. Then for any matrix $A$,

$$
\left\langle A, G_{X, \omega}(s) A\right\rangle=\sum_{k, l=1}^{n}\left(\left(e^{\omega} \frac{\lambda_{k}}{\lambda_{l}}\right)^{s}+\left(e^{\omega} \frac{\lambda_{k}}{\lambda_{l}}\right)^{1-s}\right)\left|A_{k, l}\right|^{2} .
$$

Since $b^{s}+b^{1-s}$ is non-increasing with respect to $s$, so is $G_{X, \omega}(s)$. To achieve equality, we need $e^{\omega} \frac{\lambda_{k}}{\lambda_{l}}=1$ for all $k, l$. Hence $\omega=0$ and there exists some $c=\lambda_{k}$ for all $1 \leq k \leq n$ (i.e., $X=c \mathbb{I})$.

2. From the last equation, by using the range of $b^{s}+b^{1-s}$, we immediately have

$$
\begin{aligned}
& \left\langle A, G_{X, \omega}(s) A\right\rangle \leq \sum_{k, l}\left(1+e^{\omega} \frac{\lambda_{k}}{\lambda_{l}}\right)\left|A_{k, l}\right|^{2} \leq\left(1+e^{\omega} \frac{\lambda_{\max }(X)}{\lambda_{\min }(X)}\right)\langle A, A\rangle ; \\
& \left\langle A, G_{X, \omega}(s) A\right\rangle \geq \sum_{k, l} 2 \sqrt{e^{\omega} \frac{\lambda_{k}}{\lambda_{l}}}\left|A_{k, l}\right|^{2} \geq 2 \sqrt{e^{\omega} \frac{\lambda_{\min }(X)}{\lambda_{\max }(X)}}\langle A, A\rangle,
\end{aligned}
$$

which proves part (2). 
Proof of Proposition V.8. Let us first introduce some useful notations. Suppose $\rho_{t}$ is evolving according to the Lindblad equation (1) and $\beta_{t}=1+\left(\alpha_{0}-1\right) e^{\eta 2 K t}$ is also changing with respect to time for some positive constant $\eta$, independent of any specific initial condition $\rho_{0}$. The parameter $\eta \in(0,1]$ is yet to be determined later. We are interested in the time interval $[0, T]$ such that $\left.\beta_{t}\right|_{t=0}=\alpha_{0},\left.\beta_{t}\right|_{t=T}=\alpha_{1}$. More specifically, $T=\frac{1}{2 K \eta} \log \left(\frac{\alpha_{1}-1}{\alpha_{0}-1}\right)$.

Let us define an important quantity $\rho_{\sigma, t}$ by

$$
\rho_{\sigma, t}:=\Gamma_{\sigma}^{\frac{1-\beta_{t}}{\beta_{t}}}\left(\rho_{t}\right) \text {. }
$$

We also define

$$
Z_{t}:=\operatorname{Tr}\left(\left(\Gamma_{\sigma}^{\frac{1-\beta_{t}}{\beta_{t}}}\left(\rho_{t}\right)\right)^{\beta_{t}}\right) \equiv \operatorname{Tr}\left(\rho_{\sigma, t}^{\beta_{t}}\right) \text { and } F_{t}:=\frac{1}{\beta_{t}} \log Z_{t}
$$

After taking the derivative of $F_{t}$ with respect to time $t$ and arranging terms,

$$
\beta_{t}^{2} Z_{t} \dot{F}_{t}=\beta_{t} \dot{Z}_{t}-\dot{\beta}_{t} Z_{t} \log \left(Z_{t}\right)
$$

We claim that to prove the inequality (65) it suffices to show that $F_{t}$ is monotonically decreasing, i.e., $\dot{F}_{t} \leq 0$ on the interval $[0, T]$. In fact, from the definition of $F$ and the sandwiched Rényi divergence $(3)$, we immediately obtain from $F_{T} \leq F_{0}$ that

$$
D_{\alpha_{1}}\left(\rho_{T} \| \sigma\right) \leq \frac{\alpha_{1}}{\alpha_{1}-1} \frac{\alpha_{0}-1}{\alpha_{0}} D_{\alpha_{0}}\left(\rho_{0} \| \sigma\right) \leq D_{\alpha_{0}}\left(\rho_{0} \| \sigma\right) .
$$

The rest devotes to the proof of $\dot{F}_{t} \leq 0$, which is done in the following steps.

Step (I): Simplification of $\dot{F}_{t}$.

Let us first compute $\dot{Z}_{t}$.

$$
\begin{aligned}
\dot{Z}_{t} & \equiv \frac{\mathrm{d}}{\mathrm{d} t} \operatorname{Tr}\left(\left(\sigma^{\frac{1-\beta_{t}}{2 \beta_{t}}} \rho_{t} \sigma^{\frac{1-\beta_{t}}{2 \beta_{t}}}\right)^{\beta_{t}}\right) \\
& =\beta_{t} \operatorname{Tr}\left(\rho_{\sigma, t}^{\beta_{t}-1}\left(\sigma^{\frac{1-\beta_{t}}{2 \beta_{t}}} \mathcal{L}^{\dagger}\left(\rho_{t}\right) \sigma^{\frac{1-\beta_{t}}{2 \beta_{t}}}-\frac{\dot{\beta}_{t}}{2 \beta_{t}^{2}} \sigma^{\frac{1-\beta_{t}}{2 \beta_{t}}}\left\{\log (\sigma), \rho_{t}\right\} \sigma^{\frac{1-\beta_{t}}{2 \beta_{t}}}\right)\right)+\dot{\beta}_{t} \operatorname{Tr}\left(\rho_{\sigma, t}^{\beta_{t}} \log \left(\rho_{\sigma, t}\right)\right) \\
& =\beta_{t} \operatorname{Tr}\left(\sigma^{\frac{1-\beta_{t}}{2 \beta_{t}}} \rho_{\sigma, t}^{\beta_{t}-1} \sigma^{\frac{1-\beta_{t}}{2 \beta_{t}}} \mathcal{L}^{\dagger}\left(\rho_{t}\right)\right)-\frac{\dot{\beta}_{t}}{\beta_{t}} \operatorname{Tr}\left(\rho_{\sigma, t}^{\beta_{t}} \log (\sigma)\right)+\frac{\dot{\beta}_{t}}{\beta_{t}} \operatorname{Tr}\left(\rho_{\sigma, t}^{\beta_{t}} \log \left(\rho_{\sigma, t}^{\beta_{t}}\right)\right)
\end{aligned}
$$

where the anti-commutator $\{\cdot, \cdot\}$ is defined as $\{A, B\}:=A B+B A$ for all matrices $A$ and $B$.

Hence by (68) and the fact that $\dot{\beta}_{t}>0$,

$$
\begin{aligned}
& \beta_{t}^{2} Z_{t} \dot{F}_{t} \\
& =\beta_{t}^{2} \operatorname{Tr}\left(\sigma^{\frac{1-\beta_{t}}{2 \beta_{t}}} \rho_{\sigma, t}^{\beta_{t}-1} \sigma^{\frac{1-\beta_{t}}{2 \beta_{t}}} \mathcal{L}^{\dagger}\left(\rho_{t}\right)\right)-\dot{\beta}_{t} \operatorname{Tr}\left(\rho_{\sigma, t}^{\beta_{t}} \log (\sigma)\right)+\dot{\beta}_{t} \operatorname{Tr}\left(\rho_{\sigma, t}^{\beta_{t}} \log \left(\rho_{\sigma, t}^{\beta_{t}}\right)\right)-\dot{\beta}_{t} Z_{t} \log \left(Z_{t}\right) \\
& \leq \beta_{t}^{2} \operatorname{Tr}\left(\sigma^{\frac{1-\beta_{t}}{2 \beta_{t}}} \rho_{\sigma, t}^{\beta_{t}-1} \sigma^{\frac{1-\beta_{t}}{2 \beta_{t}}} \mathcal{L}^{\dagger}\left(\rho_{t}\right)\right)+\frac{\dot{\beta}_{t}}{2 K} \operatorname{Tr}\left(-\mathcal{L}^{\dagger}\left(\rho_{\sigma, t}^{\beta_{t}}\right)\left(\log \left(\rho_{\sigma, t}^{\beta_{t}}\right)-\log (\sigma)\right)\right) \\
& =: T_{1}+\frac{\dot{\beta}_{t}}{2 K} T_{2} .
\end{aligned}
$$


In the last line, we introduce $T_{1}$ and $T_{2}$ as short-hand notations. Next, we further simplify $T_{1}$ and $T_{2}$.

For $T_{1}$

$$
\begin{aligned}
& T_{1}=\beta_{t}^{2} \operatorname{Tr}\left(\sigma^{\frac{1-\beta_{t}}{2 \beta_{t}}} \rho_{\sigma, t}^{\beta_{t}-1} \sigma^{\frac{1-\beta_{t}}{2 \beta_{t}}} \mathcal{L}^{\dagger}\left(\rho_{t}\right)\right) \\
& \stackrel{(34)}{=} \beta_{t}\left(\beta_{t}-1\right) Z_{t} \operatorname{Tr}\left(\left.\frac{\delta D_{\beta_{t}}(\rho \| \sigma)}{\delta \rho}\right|_{\rho=\rho_{t}} \mathcal{L}^{\dagger}\left(\rho_{t}\right)\right) \\
& \stackrel{(44)}{=}-\beta_{t}\left(\beta_{t}-1\right) Z_{t}\left\langle\left.\nabla \frac{\delta D_{\beta_{t}}(\rho \| \sigma)}{\delta \rho}\right|_{\rho=\rho_{t}},\left[\rho_{t}\right]_{\beta_{t}, \vec{\omega}}\left(\left.\nabla \frac{\delta D_{\beta_{t}}(\rho \| \sigma)}{\delta \rho}\right|_{\rho=\rho_{t}}\right)\right\rangle \\
& \stackrel{(36)}{=}-\left(\beta_{t}-1\right) Z_{t}^{2} \sum_{j}\left\langle A_{j},\left[\rho_{\sigma, t}^{\beta_{t}-1}\right]_{\frac{\left(\beta_{t}-1\right) \omega_{j}}{\beta_{t}}} \circ\left[\rho_{\sigma, t}\right]_{\frac{\omega_{j}}{\beta_{t}}}\left(A_{j}\right)\right\rangle,
\end{aligned}
$$

where $A_{j}=\left[\rho_{\sigma, t}^{\beta_{t}-1}\right]_{\frac{\left(\beta_{t}-1\right) \omega_{j}}{\beta_{t}}}^{-1} \circ \Gamma_{\sigma}^{\frac{\beta_{t}-1}{\beta_{t}}}\left(\left.\partial_{j} \frac{\delta D_{\beta_{t}}(\rho|| \sigma)}{\delta \rho}\right|_{\rho=\rho_{t}}\right)$.

As for $T_{2}$, by using (44) again (for the case $\alpha=1$ in (44)),

$$
\begin{aligned}
T_{2} & =-\left\langle\log \left(\rho_{\sigma, t}^{\beta_{t}}\right)-\log (\sigma), \mathcal{L}^{\dagger}\left(\rho_{\sigma, t}^{\beta_{t}}\right)\right\rangle \\
& =\left\langle\nabla\left(\beta_{t} \log \left(\rho_{\sigma, t}\right)-\log (\sigma)\right),\left[\rho_{\sigma, t}^{\beta_{t}}\right]_{\vec{\omega}} \nabla\left(\beta_{t} \log \left(\rho_{\sigma, t}\right)-\log (\sigma)\right)\right\rangle .
\end{aligned}
$$

Consider the term

$$
\begin{aligned}
& \partial_{j}\left(\beta_{t} \log \left(\rho_{\sigma, t}\right)-\log (\sigma)\right) \\
&= \beta_{t}\left(\partial_{j} \log \left(\rho_{\sigma, t}\right)-\frac{\omega_{j}}{\beta_{t}} V_{j}\right) \\
&= \beta_{t}\left(V_{j} \log \left(e^{-\frac{\omega_{j}}{2 \beta_{t}}} \rho_{\sigma, t}\right)-\log \left(e^{\frac{\omega_{j}}{2 \beta_{t}}} \rho_{\sigma, t}\right) V_{j}\right) \\
& \stackrel{(45)}{=} Z_{t}\left(\left[\rho_{\sigma, t}^{\beta_{t}-1}\right]_{\frac{\beta_{t}-1}{\beta_{t}} \omega_{j}}^{-1} \circ \Gamma_{\sigma}^{\frac{\beta_{t}-1}{\beta_{t}}}\right) \partial_{j}\left(\left.\frac{\delta D_{\beta_{t}}(\rho \| \sigma)}{\delta \rho}\right|_{\rho=\rho_{t}}\right) \\
&=Z_{t} A_{j} .
\end{aligned}
$$

Here, to obtain the second line, we have used $\partial_{j} \log (\sigma) \equiv\left[V_{j}, \log (\sigma)\right]=\left.\partial_{s}\left(\Delta_{\sigma}^{-s} V_{j}\right)\right|_{s=0}=$ $\left.\partial_{s}\left(e^{\omega_{j} s} V_{j}\right)\right|_{s=0}=\omega_{j} V_{j}$ (see [18, Lemma 5.9]). Then, it follows immediately that

$$
T_{2}=Z_{t}^{2} \sum_{j}\left\langle A_{j},\left[\rho_{\sigma, t}^{\beta_{t}}\right]_{\omega_{j}}\left(A_{j}\right)\right\rangle .
$$

By plugging the expressions of $T_{1}$ and $T_{2}$ back into (72), and using the fact that $\frac{\dot{\beta}_{t}}{2 K}=$ $\frac{\eta 2 K\left(\alpha_{0}-1\right) e^{\eta 2 K t}}{2 K}=\eta\left(\beta_{t}-1\right)$, we have

$$
\beta_{t}^{2} Z_{t} \dot{F}_{t} \leq\left(\beta_{t}-1\right) Z_{t}^{2} \sum_{j}\left(\eta\left\langle A_{j},\left[\rho_{\sigma, t}^{\beta_{t}}\right]_{\omega_{j}}\left(A_{j}\right)\right\rangle-\left\langle A_{j},\left[\rho_{\sigma, t}^{\beta_{t}-1}\right]_{\frac{\left(\beta_{t}-1\right) \omega_{j}}{\beta_{t}}} \circ\left[\rho_{\sigma, t}\right]_{\frac{\omega_{j}}{\beta_{t}}}\left(A_{j}\right)\right\rangle\right) .
$$


Step (II): We show that $\dot{F}_{t} \leq 0$.

By observing the last equation, since $\beta_{t}-1>0$ and $Z_{t}>0$, it is then sufficient to show that each term indexed by $j$ on the right hand side of the last equation is less than or equal to zero, namely,

$$
\eta\left\langle A_{j},\left[\rho_{\sigma, t}^{\beta_{t}}\right]_{\omega_{j}}\left(A_{j}\right)\right\rangle \leq\left\langle A_{j},\left[\rho_{\sigma, t}^{\beta_{t}-1}\right]_{\frac{\left(\beta_{t}-1\right) \omega_{j}}{\beta_{t}}} \circ\left[\rho_{\sigma, t}\right]_{\frac{\omega_{j}}{\beta_{t}}}\left(A_{j}\right)\right\rangle,
$$

for any time $t \in[0, T]$ and index $j$. In the commutative case, both left and right hand sides represent multiplication of the operator $\rho_{\sigma, t}^{\beta_{t}}$ and the above inequality holds trivially. For noncommutative (quantum) systems, the above inequality is non-trivial. In this step, we will simplify the expression in the last equation and furthermore find sufficient conditions for $\eta$ so that the last inequality holds.

By definition (21) and let $B_{j}=A_{j} \frac{\beta_{t}}{\frac{2}{2}}$, the above inequality can be simplified to

$$
\begin{aligned}
& \eta\left\langle B_{j}, \int_{0}^{1} e^{\omega_{j} s} \rho_{\sigma, t}^{\beta_{t} s} B_{j} \rho_{\sigma, t}^{-\beta_{t} s} \mathrm{~d} s\right\rangle \\
\leq & \left\langle B_{j}, \int_{0}^{1} \int_{0}^{1} e^{\frac{\left(\beta_{t}-1\right) \omega_{j} v}{\beta_{t}}} e^{\frac{\omega_{j} u}{\beta_{t}}} \rho_{\sigma, t}^{\left(\beta_{t}-1\right) v+u} B_{j} \rho_{\sigma, t}^{-\left(\beta_{t}-1\right) v-u} \mathrm{~d} u \mathrm{~d} v\right\rangle \\
= & \left\langle B_{j}, \int_{0}^{1} e^{\omega_{j} s} \rho_{\sigma, t}^{\beta_{t} s} B_{j} \rho_{\sigma, t}^{-\beta_{t} s}\left(\int_{U_{s}}\left|\frac{\partial(u, v)}{\partial(s, \widetilde{s})}\right| \mathrm{d} \widetilde{s}\right) \mathrm{d} s\right\rangle \\
= & \left\langle B_{j}, \int_{0}^{1} e^{\omega_{j} s} \rho_{\sigma, t}^{\beta_{t} s} B_{j} \rho_{\sigma, t}^{-\beta_{t} s} f(s) \mathrm{d} s\right\rangle .
\end{aligned}
$$

From the second line to the third line, we have used the change of variable $s=\frac{\left(\beta_{t}-1\right) v+u}{\beta_{t}}$ and $\widetilde{s}=\frac{\left(\beta_{t}-1\right) v-u}{\beta_{t}}$. The set $U_{s}$ is defined as

$$
U_{s}:=\left\{\tilde{s} \in \mathbb{R}: 0 \leq \frac{\beta_{t}}{2\left(\beta_{t}-1\right)}(\widetilde{s}+s) \leq 1 \text { and } 0 \leq \frac{\beta_{t}}{2}(s-\widetilde{s}) \leq 1\right\}
$$

The weight function $f(s):=\int_{U_{s}}\left|\frac{\partial(u, v)}{\partial(s, \widetilde{s})}\right| \mathrm{d} \widetilde{s}=\int_{U_{s}} \frac{\beta_{t}^{2}}{2\left(\beta_{t}-1\right)} \mathrm{d} \widetilde{s}$ is a probability distribution on $[0,1]$ which can be expressed explicitly as

$$
f(s)=\frac{\beta_{t}^{2}}{2\left(\beta_{t}-1\right)}\left(\min \left(s, \frac{2\left(\beta_{t}-1\right)}{\beta_{t}}-s\right)-\max \left(-s, s-\frac{2}{\beta_{t}}\right)\right), \quad s \in[0,1] .
$$

The graphs of $f(s)$ for $\beta_{t}=1.5,2$, and 3 are given in Figure 2 for illustration. The largest value of $f(s)$ on the interval $[0,1]$ is $\beta_{t}$ if $\beta_{t} \leq 2$, and is $\frac{\beta_{t}}{\beta_{t}-1}$ if $\beta_{t} \geq 2$. In either case, since $\beta_{t}>1$, the largest value of $f(s)$ must be strictly larger than 1 . Therefore, we can define $s_{1}$ and $s_{2}$ as the two zeros of the function $f(s)-1$, as visualized in the figure. More explicitly, $s_{1}=\left(\beta_{t}-1\right) / \beta_{t}^{2}$ and by symmetry, $s_{2}=1-s_{1}$.

To show (74), it is then sufficient to show that

$$
\eta \int_{0}^{1} e^{\omega_{j} s} \rho_{\sigma, t}^{\beta_{t} s}(\cdot) \rho_{\sigma, t}^{-\beta_{t} s} \mathrm{~d} s \leq \int_{0}^{1} e^{\omega_{j} s} \rho_{\sigma, t}^{\beta_{t} s}(\cdot) \rho_{\sigma, t}^{-\beta_{t} s} f(s) \mathrm{d} s,
$$




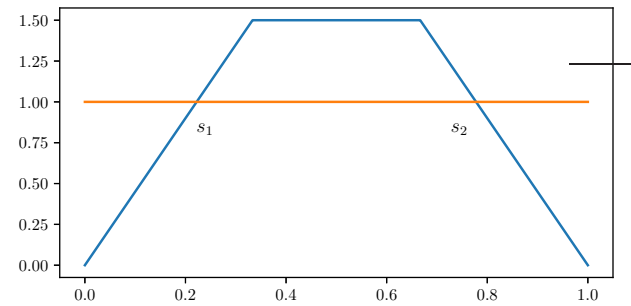

(a) $\beta_{t}=1.5$

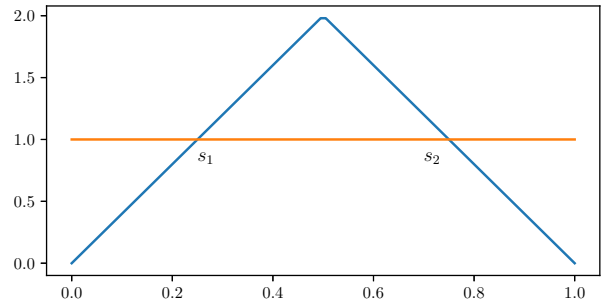

(b) $\beta_{t}=2$

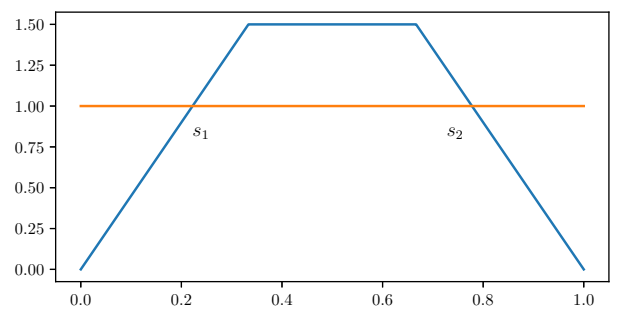

(c) $\beta_{t}=3$

FIG. 2: Weight function $f(s)$ in blue color for $\beta_{t}=1.5,2,3$.

in the sense of operators. In the above inequality, the notation $\rho_{\sigma, t}^{\beta_{t} s}(\cdot) \rho_{\sigma, t}^{-\beta_{t} s}$ is an operator mapping $A \in \mathfrak{M}$ to $\rho_{\sigma, t}^{\beta_{t} s} A \rho_{\sigma, t}^{-\beta_{t} s} \in \mathfrak{M}$. Notice that the function $f(s)$ is symmetric with respect to the axis $s=1 / 2$ and recall the definition of $G_{X, \omega}(s)$ given by (69). We can rewrite the last inequality as

$$
\eta \int_{0}^{1 / 2} G_{\rho_{\sigma, t}^{\beta_{t}, \omega_{j}}}(s) \mathrm{d} s \leq \int_{0}^{1 / 2} G_{\rho_{\sigma, t}^{\beta_{t}, \omega_{j}}}(s) f(s) \mathrm{d} s .
$$

By Lemma V.11 part (1), we have

$$
\int_{0}^{1 / 2} G_{\rho_{\sigma, t}^{\beta_{t}, \omega_{j}}}(s) \mathrm{d} s \geq \int_{0}^{1 / 2} G_{\rho_{\sigma, t}^{\beta_{t}, \omega_{j}}}(s) f(s) \mathrm{d} s .
$$

Apparently we need $\eta \leq 1$ for the inequality (76) to hold. Thus from now on we shall restrict to $\eta \leq 1$. By subtracting the common parts in the integral (76), in order to show (76), it suffices to prove that

$$
\eta \int_{0}^{s_{1}} G_{\rho_{\sigma, t}^{\beta_{t}, \omega_{j}}}(s)(1-f(s)) \mathrm{d} s \leq \int_{s_{1}}^{\frac{1}{2}} G_{\rho_{\sigma, t}^{\beta_{t}}, \omega_{j}}(s)(f(s)-1) \mathrm{d} s .
$$

Note that $\int_{0}^{s_{1}}(1-f(s)) \mathrm{d} s=\int_{s_{1}}^{\frac{1}{2}}(f(s)-1) \mathrm{d} s$ since $f(s)$ is symmetric about the line $s=\frac{1}{2}$ and also $f(s)$ is a probability distribution on $[0,1]$. Then by Lemma V.11 part (1),

$$
\int_{s_{1}}^{\frac{1}{2}} G_{\rho_{\sigma, t}^{\beta_{t},}, \omega_{j}}(s)(f(s)-1) \mathrm{d} s \geq \int_{s_{1}}^{\frac{1}{2}} G_{\rho_{\sigma, t}^{\beta_{t}}, \omega_{j}}\left(\frac{1}{2}\right)(f(s)-1) \mathrm{d} s=\int_{0}^{s_{1}} G_{\rho_{\sigma, t}^{\beta_{t}}, \omega_{j}}\left(\frac{1}{2}\right)(1-f(s)) \mathrm{d} s .
$$


By Lemma V.11 part (2), if we choose $\eta$ such that

$$
\eta \leq \frac{2 \sqrt{e^{\omega_{j}} \frac{\lambda_{\min }\left(\rho_{\sigma, t}^{\beta,}\right)}{\lambda_{\max }\left(\rho_{\sigma, t}^{\beta, t}\right)}}}{1+e^{\omega_{j}} \frac{\lambda_{\max }\left(\rho_{\sigma, t)}^{\beta, t}\right)}{\lambda_{\min }\left(\rho_{\sigma, t}^{\beta, t}\right)}}
$$

then $\eta G_{\rho_{\sigma, t}^{\beta_{t}, \omega_{j}}}(s) \leq G_{\rho_{\sigma, t}^{\beta_{t}, \omega_{j}}}\left(\frac{1}{2}\right)$ for all $0 \leq s \leq \frac{1}{2}$, which implies (77).

Hence, the remaining task is to show the existence of $\eta$ such that (78) holds, for all $j \in$ $\{1,2, \cdots, J\}$, all $t \in[0, T]$ and any initial condition $\rho_{0}$ satisfying the assumption that $D\left(\rho_{0} \| \sigma\right) \leq \epsilon$ for some $0<\epsilon<\frac{\lambda_{\min }(\sigma)^{2}}{2}$; step (III) below is fully devoted into finding such an $\eta$.

Step (III): We show there exists $\eta \in(0,1]$ satisfying (78).

By observing the term on the right hand side of (78), it suffices to show that $\frac{\lambda_{\max }\left(\rho_{\sigma, t}^{\beta}\right)}{\lambda_{\min }\left(\rho_{\sigma, t}^{\beta, t}\right)}$ is bounded from above. We first establish an upper bound for $\lambda_{\max }\left(\rho_{\sigma, t}^{\beta_{t}}\right)$. In fact, from the quantum LSI (5) we have

$$
D\left(\rho_{t} \| \sigma\right) \leq D\left(\rho_{0} \| \sigma\right) e^{-2 K t} \leq \epsilon e^{-2 K t}=: \epsilon_{t} .
$$

Moreover, by the quantum Pinsker's inequality (67), we obtain that

$$
\operatorname{Tr}\left|\rho_{t}-\sigma\right| \leq \sqrt{2 \epsilon_{t}}
$$

which implies that

$$
-\sqrt{2 \epsilon_{t}} \mathbb{I} \leq \rho_{t}-\sigma \leq \sqrt{2 \epsilon_{t}} \mathbb{I}
$$

As a result,

$$
\begin{aligned}
\sigma^{\frac{1-\beta_{t}}{2 \beta_{t}}} \rho_{t} \sigma^{\frac{1-\beta_{t}}{2 \beta_{t}}} & =\sigma^{\frac{1-\beta_{t}}{2 \beta_{t}}}\left(\rho_{t}-\sigma\right) \sigma^{\frac{1-\beta_{t}}{2 \beta_{t}}}+\sigma^{\frac{1}{\beta_{t}}} \\
& \leq \sigma^{\frac{1}{\beta_{t}}}+\sqrt{2 \epsilon_{t}} \sigma^{\frac{1-\beta_{t}}{\beta_{t}}} \\
& \leq\left(\lambda_{\max }(\sigma)^{\frac{1}{\beta_{t}}}+\sqrt{2 \epsilon_{t}} \lambda_{\min }(\sigma)^{\frac{1-\beta_{t}}{\beta_{t}}}\right) \mathbb{I} .
\end{aligned}
$$

It follows that

$$
\begin{aligned}
\lambda_{\max }\left(\rho_{\sigma, t}^{\beta_{t}}\right) & =\lambda_{\max }\left(\left(\sigma^{\frac{1-\beta_{t}}{2 \beta_{t}}} \rho_{t} \sigma^{\frac{1-\beta_{t}}{2 \beta_{t}}}\right)^{\beta_{t}}\right) \leq\left(\lambda_{\max }(\sigma)^{\frac{1}{\beta_{t}}}+\sqrt{2 \epsilon_{t}} \lambda_{\min }(\sigma)^{\frac{1-\beta_{t}}{\beta_{t}}}\right)^{\beta_{t}} \\
& =\lambda_{\max }(\sigma)\left(1+\frac{\sqrt{2 \epsilon_{t}}}{\lambda_{\min }(\sigma)}\left(\frac{\lambda_{\min }(\sigma)}{\lambda_{\max }(\sigma)}\right)^{\frac{1}{\beta_{t}}}\right)^{\beta_{t}} \\
& \leq \lambda_{\max }(\sigma) \exp \left(\beta_{t} \frac{\sqrt{2 \epsilon_{t}}}{\lambda_{\min }(\sigma)}\left(\frac{\lambda_{\min }(\sigma)}{\lambda_{\max }(\sigma)}\right)^{\frac{1}{\beta_{t}}}\right)
\end{aligned}
$$

In the last inequality we used an elementary inequality $(1+x)^{y}=\exp (y \log (1+x)) \leq \exp (x y)$ for $x, y \geq 0$. By the definition of $\beta_{t}$, the exponent on the right side of the above equation can be 
bounded above by

$$
\begin{aligned}
& \beta_{t} \frac{\sqrt{2 \epsilon_{t}}}{\lambda_{\min }(\sigma)}\left(\frac{\lambda_{\min }(\sigma)}{\lambda_{\max }(\sigma)}\right)^{\frac{1}{\beta_{t}}} \\
= & \left(1+\left(\alpha_{0}-1\right) e^{\eta 2 K t}\right) \frac{\sqrt{2 \epsilon}}{\lambda_{\min }(\sigma)} \exp \left(-K t-\frac{1}{\beta_{t}} \log \left(\frac{\lambda_{\max }(\sigma)}{\lambda_{\min }(\sigma)}\right)\right) \\
\leq & \frac{\sqrt{2 \epsilon}}{\lambda_{\min }(\sigma)}+\left(\alpha_{0}-1\right) \frac{\sqrt{2 \epsilon}}{\lambda_{\min }(\sigma)} \exp \left(\eta 2 K t-K t-\frac{1}{\beta_{t}} \log \left(\frac{\lambda_{\max }(\sigma)}{\lambda_{\min }(\sigma)}\right)\right) .
\end{aligned}
$$

If we restrict $\eta \leq \frac{1}{2}$, then the quantity above is less than $\alpha_{0} \frac{\sqrt{2 \epsilon}}{\lambda_{\min }(\sigma)}$ and thus

$$
\lambda_{\max }\left(\rho_{\sigma, t}^{\beta_{t}}\right) \leq \lambda_{\max }(\sigma) \exp \left(\alpha_{0} \frac{\sqrt{2 \epsilon}}{\lambda_{\min }(\sigma)}\right) .
$$

Next, we prove a lower bound for $\lambda_{\min }\left(\rho_{\sigma, t}^{\beta, t}\right)$. In fact, similar to (79), one can show that

$$
\begin{aligned}
\sigma^{\frac{1-\beta_{t}}{2 \beta_{t}}} \rho_{t} \sigma^{\frac{1-\beta_{t}}{2 \beta_{t}}} & =\sigma^{\frac{1-\beta_{t}}{2 \beta_{t}}}\left(\rho_{t}-\sigma\right) \sigma^{\frac{1-\beta_{t}}{2 \beta_{t}}}+\sigma^{\frac{1}{\beta_{t}}} \geq \sigma^{\frac{1}{\beta_{t}}}-\sqrt{2 \epsilon_{t}} \sigma^{\frac{1-\beta_{t}}{\beta_{t}}} \\
& \geq\left(\lambda_{\min }(\sigma)^{\frac{1}{\beta_{t}}}-\sqrt{2 \epsilon_{t}} \lambda_{\min }(\sigma)^{\frac{1-\beta_{t}}{\beta_{t}}}\right) \mathbb{I}=\lambda_{\min }(\sigma)^{\frac{1}{\beta_{t}}}\left(1-\frac{\sqrt{2 \epsilon_{t}}}{\lambda_{\min }(\sigma)}\right) \mathbb{I}>0,
\end{aligned}
$$

and hence

$$
\begin{aligned}
\lambda_{\min }\left(\rho_{\sigma, t}^{\beta_{t}}\right) & =\lambda_{\min }\left(\left(\sigma^{\frac{1-\beta_{t}}{2 \beta_{t}}} \rho_{t} \sigma^{\frac{1-\beta_{t}}{2 \beta_{t}}}\right)^{\beta_{t}}\right) \geq \lambda_{\min }(\sigma)\left(1-\frac{\sqrt{2 \epsilon_{t}}}{\lambda_{\min }(\sigma)}\right)^{\beta_{t}} \\
& =\lambda_{\min }(\sigma) \exp \left(\beta_{t} \log \left(1-\frac{\sqrt{2 \epsilon}}{\lambda_{\min }(\sigma)} e^{-K t}\right)\right) .
\end{aligned}
$$

We show that the exponent on the right side of the above equation can be bounded from below. Indeed, since $1-\frac{\sqrt{2 \epsilon}}{\lambda_{\min }(\sigma)} e^{-K t} \geq 1-\frac{\sqrt{2 \epsilon}}{\lambda_{\min }(\sigma)}>0$ and $\log (1-x) \geq-x /(1-x)$ for $x \in[0,1)$, if we restrict $\eta \leq 1 / 2$, then

$$
\begin{aligned}
0 & >\beta_{t} \log \left(1-\frac{\sqrt{2 \epsilon}}{\lambda_{\min }(\sigma)} e^{-K t}\right)=\left(1+\left(\alpha_{0}-1\right) e^{\eta 2 K t}\right) \log \left(1-\frac{\sqrt{2 \epsilon}}{\lambda_{\min }(\sigma)} e^{-K t}\right) \\
& \geq \log \left(1-\frac{\sqrt{2 \epsilon}}{\lambda_{\min }(\sigma)}\right)-\left(\alpha_{0}-1\right) e^{\eta 2 K t} \frac{\frac{\sqrt{2 \epsilon}}{\lambda_{\min }(\sigma)} e^{-K t}}{1-\frac{\sqrt{2 \epsilon}}{\lambda_{\min }(\sigma)} e^{-K t}} \\
& =\log \left(1-\frac{\sqrt{2 \epsilon}}{\lambda_{\min }(\sigma)}\right)-\left(\alpha_{0}-1\right) e^{\eta 2 K t-K t} \frac{\frac{\sqrt{2 \epsilon}}{\lambda_{\min }(\sigma)}}{1-\frac{\sqrt{2 \epsilon}}{\lambda_{\min }(\sigma)}} \\
& \geq \log \left(1-\frac{\sqrt{2 \epsilon}}{\lambda_{\min }(\sigma)}\right)-\left(\alpha_{0}-1\right) \frac{\frac{\sqrt{2 \epsilon}}{\lambda_{\min }(\sigma)}}{1-\frac{\sqrt{2 \epsilon}}{\lambda_{\min }(\sigma)}} \geq-\alpha_{0} \frac{\frac{\sqrt{2 \epsilon}}{\lambda_{\min }(\sigma)}}{1-\frac{\sqrt{2 \epsilon}}{\lambda_{\min }(\sigma)}} .
\end{aligned}
$$


This implies that

$$
\lambda_{\min }\left(\rho_{\sigma, t}^{\beta_{t}}\right) \geq \lambda_{\min }(\sigma) \exp \left(-\alpha_{0} \frac{\frac{\sqrt{2 \epsilon}}{\lambda_{\min (\sigma)}}}{1-\frac{\sqrt{2 \epsilon}}{\lambda_{\min }(\sigma)}}\right) .
$$

Combining the estimations (80) and (81) yields

$$
\begin{aligned}
\frac{\lambda_{\max }\left(\rho_{\sigma, t}^{\beta_{t}}\right)}{\lambda_{\min }\left(\rho_{\sigma, t}^{\beta_{t}}\right)} & \leq \frac{\lambda_{\max }(\sigma) \exp \left(\alpha_{0} \frac{\sqrt{2 \epsilon}}{\lambda_{\min }(\sigma)}\right)}{\lambda_{\min }(\sigma) \exp \left(-\alpha_{0} \frac{\frac{\sqrt{2 \epsilon}}{\lambda_{\min }(\sigma)}}{1-\frac{\sqrt{2 \epsilon}}{\lambda_{\min }(\sigma)}}\right)} \\
& =\frac{\lambda_{\max }(\sigma)}{\lambda_{\min }(\sigma)} \exp \left(\alpha_{0} \sqrt{2 \epsilon} \frac{2 \lambda_{\min }(\sigma)-\sqrt{2 \epsilon}}{\lambda_{\min }(\sigma)\left(\lambda_{\min }(\sigma)-\sqrt{2 \epsilon}\right)}\right)=: \Lambda .
\end{aligned}
$$

This leads to

$$
\frac{2 \sqrt{e^{\omega_{j}} \frac{\lambda_{\min }\left(\rho_{\sigma, t}^{\beta_{t}}\right)}{\lambda_{\max }\left(\rho_{\sigma, t}^{\beta_{t}}\right)}}}{1+e^{\omega_{j}} \frac{\lambda_{\max }\left(\rho_{\sigma, t}^{\left.\beta_{t}\right)}\right.}{\lambda_{\min }\left(\rho_{\sigma, t}^{\beta_{t}}\right)}} \geq \frac{2 \sqrt{e^{\omega_{j}} \frac{1}{\Lambda}}}{1+e^{\omega_{j}} \Lambda} .
$$

This finishes the proof of (78) with

$$
\eta:=\min \left(\frac{1}{2}, \min _{j=1}^{\lrcorner}\left\{\frac{2 \sqrt{e^{\omega_{j}} \frac{1}{\Lambda}}}{1+e^{\omega_{j}} \Lambda}\right\}\right)>0 .
$$

\section{F. Discussion on quantum comparison theorem}

Finally, we would like to comment on the quantum comparison theorem (see Proposition V.8).

Discussion on its proof and the hypercontractivity: The main idea of proving Proposition V.8 is to verify the hypercontractivity in the sense of noncommutative $\mathbb{L}_{\alpha}$ space (see e.g., [45, Definition 12]) under the assumption of the quantum LSI. More specifically, suppose $\beta_{t}=1+\left(\alpha_{0}-1\right) e^{\eta 2 K t}$ for some constant $\eta>0$ and $\rho_{t}$ follows the Lindblad equation, i.e., $\dot{\rho}_{t}=\mathcal{L}^{\dagger}\left(\rho_{t}\right)$, then for the time interval $[0, T]$, where $T=\frac{1}{2 K \eta} \log \left(\frac{\alpha_{1}-1}{\alpha_{0}-1}\right)$, we need to show that

$$
\left[\operatorname{Tr}\left(\left(\Gamma_{\sigma}^{\frac{1-\beta_{t}}{\beta_{t}}}\left(\rho_{t}\right)\right)^{\beta_{t}}\right)\right]^{\frac{1}{\beta_{t}}} \text { is a non-increasing function of time } t \in[0, T] .
$$

The function $F_{t}$ in the last subsection is simply the logarithm of this function. To see why this requirement (83) is closely related to the hypercontractivity in the noncommutative $\mathbb{L}_{\alpha}$ spaces, let us introduce the relative density $X_{t}=\Gamma_{\sigma}^{-1}\left(\rho_{t}\right)$; the time-evolution of the relative density $X_{t}$ has the generator $\mathcal{L}$, i.e., $X_{t}=e^{t \mathcal{L}}\left(X_{0}\right)$, which can be directly verified with the help of (12). With these 
notations and definitions, (83) means $\left\|e^{t \mathcal{L}}\left(X_{0}\right)\right\|_{\beta_{t}, \sigma} \leq\left\|X_{0}\right\|_{\alpha_{0}, \sigma}$, which has the same form as [45, Definition 12]. In a recent paper [46], for primitive Lindblad equations with GNS-detailed balance, a quantum Stroock-Varopoulos inequality (see [46, Theorem 14]) has been proved, whose implication (see [46, Corollary 17]) shows that one can pick $\eta_{S V I}=2 \kappa_{2} / K$ (see (28) for the definition of $\kappa_{2}$ ); the subscript $S V I$ is used to emphasize using the quantum Stroock-Varopoulos inequality. Recall that $K=2 \kappa_{1}$ (31); the quantum Stroock-Varopoulos inequality shows that $\kappa_{1} \geq \kappa_{2}$ (see also Theorem II.10), hence $\eta_{S V I} \leq 1$, which is consistent with our observation in the proof that $\eta \leq 1$. We make some comments on the difference between Proposition V.8 and the above estimation using the quantum Stroock-Varopoulos inequality in [46]:

1. Using the estimation from the quantum Stroock-Varopoulos inequality, the prefactor $\frac{1}{2 K \eta}$ in (66), is simply $\frac{1}{4 \kappa_{2}}$; in this paper, we directly use $K / 2 \equiv \kappa_{1}$ and the information about the spectrum of $\sigma$.

2. There is, however, a more stringent assumption on the initial condition $\rho_{0}$ in Proposition V.8. Because of such a restriction on initial conditions, even though our proof is related to verify the hypercontractivity, strictly speaking, we haven't really proved the hypercontractivity by solely assuming the quantum LSI (5) for $\alpha=1$.

3. Despite the restriction on initial conditions $\rho_{0}$, our proof is probably more similar to the classical result for the Fokker-Planck equation case in [28, Lemma 3.4]. Moreover, our proof for the quantum comparison theorem does not require any prior knowledge about noncommutative $\mathbb{L}_{\alpha}$ spaces.

Comparison with the classical result: By comparing Proposition V.8 and a similar result for the Fokker-Planck equation (c.f. [28, Lemma 3.4]), it appears that the waiting time $T$ in Proposition V.8 is much larger than that for the Fokker-Planck equation. More specifically, the parameter $\eta \leq 1$ for the Lindblad equation, and $\eta=1$ for the Fokker-Planck equation.

\section{CONCLUSION AND DISCUSSIONS}

In this paper, we have extended the gradient flow structure of the quantum relative entropy in [18] to sandwiched Rényi divergences of any order $\alpha \in(0, \infty)$, for primitive Lindblad equations with GNS-detailed balance. The necessary condition for the validity of such a gradient flow structure has been briefly discussed. Furthermore, we have proved the exponential decay of the sandwiched Rényi divergence of any order $\alpha \in(0, \infty)$. We conclude with some remarks on open questions and future research directions.

1. (More general entropy). In this paper, we have considered the entropy production of the sandwiched Rényi divergence, which is a generalization of the quantum relative entropy. One natural question is whether the gradient flow structure and exponential decay proved in this paper for the sandwiched Rényi divergence can be extended to the more general $(\alpha, z)$-Rényi divergence $[41,42]$, for certain ranges of $(\alpha, z)$.

2. (Gap between sufficient and necessary conditions for having a gradient flow structure). We have mentioned in Sec. IV that there is still a gap between sufficient and necessary conditions to regard Lindblad equations as the gradient flow dynamics of sandwiched Rényi divergences (including the quantum relative entropy [19]). Characterizing the QMS with various detailed balance conditions, beyond GNS-detailed balance, might be important to resolve this issue, 
i.e., studying different versions of Theorem II.1 by considering various detailed balance assumptions.

3. (Quantum Wasserstein distance). In the classical optimal transport theory, Wasserstein distance, introduced to capture the cost of transportation between two probability measures, has been widely studied [54]. Due to its success for classical systems, it is a natural question to explore the notion quantum Wasserstein distance. There are many attempts to define this concept, via the Monge formalism [55, 56], the Kantorovich formalism [57-59] and the Benamou-Brenier formalism [17, 18, 33,60-63]. The one that is most relevant to this paper is Benamou-Brenier formalism [64], which offers a dynamical picture to view the Wasserstein distance between any two states $\rho_{0}$ and $\rho_{1}$ as the minimal Lagrangian along all possible paths connecting $\rho_{0}$ and $\rho_{1}$. This Lagrangian could possibly be defined via metric tensors in Riemannian manifolds. In [18, Sec. 8], with the variational formalism of primitive Lindblad equations with GNS-detailed balance for the quantum relative entropy, quantum Wasserstein distance is defined via Benamou-Brenier formalism. With the generalization of this variational formalism to the case of the sandwiched Rényi divergence, one could straightforwardly generalize the quantum Wasserstein distance defined in [18] to a quantum $(\alpha, q)$-Wasserstein distance.

Definition VI.1 (Quantum $(\alpha, q)$-Wasserstein distance). For any order $\alpha \in(0, \infty)$ and power $q \geq 1$, a quantum $(\alpha, q)$-Wasserstein distance is defined in the following way: for any $\rho_{0}, \rho_{1} \in \mathfrak{D}$,

$$
W_{\alpha, q}\left(\rho_{0}, \rho_{1}\right):=\inf _{\gamma(s)}\left(\int_{0}^{1}\left(g_{\alpha, \gamma(s), \mathcal{L}}(\dot{\gamma}(s), \dot{\gamma}(s))\right)^{\frac{q}{2}} \mathrm{~d} s\right)^{\frac{1}{q}}
$$

where the infimum is taken over all smooth paths $\gamma(\cdot):[0,1] \rightarrow \mathfrak{D}_{+}$that connect $\rho_{0}$ and $\rho_{1}$; more specifically, $\gamma(0)=\rho_{0}, \gamma(1)=\rho_{1}$ and $\rho(s) \in \mathfrak{D}_{+}$for all $s \in(0,1)$.

The study of the properties of this quantum Wasserstein distance is also an interesting topic for future works.

4. (Lindblad equation with energy-conservation term). In general, Lindblad equation has both energy-conservation term and dissipative term, i.e., it has the form

$$
\dot{\rho}_{t}=\mathcal{L}^{\dagger}\left(\rho_{t}\right)=-i\left[H, \rho_{t}\right]+\sum_{j} c_{j}\left(\left[V_{j} \rho_{t}, V_{j}^{*}\right]+\left[V_{j}, \rho_{t} V_{j}^{*}\right]\right),
$$

where $c_{j}$ are non-negative constants. The Hamiltonian $H$ in general does not vanish $[29,30]$. Therefore, it is also an interesting question to explore how we could generalize results in the present paper to such general Lindblad equations with non-trivial $H$.

An exact construction of gradient flow structure in a Riemannian manifold (see Sec. II A) does not seem to be possible, even for classical kinetic Fokker-Planck equations. However, for a generalized Kramers equation (probably regarded as the classical analog of Lindblad equations with a nontrivial Hamiltonian term), some JKO schemes have already existed in [65]. As far as we know, currently, there is no any type of JKO scheme for Lindblad equations, which is probably also an interesting question to study, due to the current active research on quantum Wasserstein distance, as we mentioned above. 
As for convergence rate to the equilibrium, there is another approach known as hypocoercivity (see e.g. [66]), for kinetic Fokker-Planck equations. We are curious about whether this approach admits a quantum extension to help study the convergence of Lindblad equations with non-trivial Hamiltonian term $H$; however, this is far beyond the scope of this paper.

Acknowledgment. This work is partially supported by the National Science Foundation under grant DMS-1454939. We thank Iman Marvian and Henry Pfister for helpful discussions.

[1] Herbert Spohn, "Entropy production for quantum dynamical semigroups," Journal of Mathematical Physics 19, 1227-1230 (1978).

[2] F. Benatti and H. Narnhofer, "Entropy behaviour under completely positive maps," Letters in Mathematical Physics 15, 325-334 (1988).

[3] Heinz-Peter Breuer, "Quantum jumps and entropy production," Phys. Rev. A 68, 032105 (2003).

[4] Franco Fagnola and Rolando Rebolledo, "Entropy production for quantum Markov semigroups," Communications in Mathematical Physics 335, 547-570 (2015).

[5] Alexander Müller-Hermes, Daniel Stilck França, and Michael M. Wolf, "Entropy production of doubly stochastic quantum channels," Journal of Mathematical Physics 57, 022203 (2016).

[6] A. Arnold, J. A. Carrillo, L. Desvillettes, J. Dolbeault, A. Jüngel, C. Lederman, P. A. Markowich, G. Toscani, and C. Villani, "Entropies and equilibria of many-particle systems: An essay on recent research," Monatsh. Math. 142, 35-43 (2004).

[7] Göran Lindblad, "Completely positive maps and entropy inequalities," Communications in Mathematical Physics 40, 147-151 (1975).

[8] Rupert L. Frank and Elliott H. Lieb, "Monotonicity of a relative Rényi entropy," Journal of Mathematical Physics 54, 122201 (2013).

[9] Alexander Müller-Hermes and David Reeb, "Monotonicity of the quantum relative entropy under positive maps," Annales Henri Poincaré 18, 1777-1788 (2017).

[10] Thomas M Cover and Joy A Thomas, Elements of information theory (John Wiley \& Sons, 2012).

[11] Fernando Brandão, Michał Horodecki, Nelly Ng, Jonathan Oppenheim, and Stephanie Wehner, "The second laws of quantum thermodynamics," Proceedings of the National Academy of Sciences 112, 3275-3279 (2015).

[12] R. Jordan, D. Kinderlehrer, and F. Otto, "The variational formulation of the Fokker-Planck equation," SIAM Journal on Mathematical Analysis 29, 1-17 (1998).

[13] Felix Otto, "The geometry of dissipative evolution equations: The porous medium equation," Communications in Partial Differential Equations 26, 101-174 (2001).

[14] Matthias Erbar, "The heat equation on manifolds as a gradient flow in the Wasserstein space," Annales de l'Institut Henri Poincaré, Probabilités et Statistiques 46, 1-23 (2010).

[15] Jan Maas, "Gradient flows of the entropy for finite Markov chains," Journal of Functional Analysis 261, 2250 - 2292 (2011).

[16] Matthias Erbar and Jan Maas, "Gradient flow structures for discrete porous medium equations," Discrete \& Continuous Dynamical Systems - A 34, 1355-1374 (2014).

[17] Eric A. Carlen and Jan Maas, "An analog of the 2-Wasserstein metric in non-commutative probability under which the Fermionic Fokker-Planck equation is gradient flow for the entropy," Communications in Mathematical Physics 331, 887-926 (2014).

[18] Eric A. Carlen and Jan Maas, "Gradient flow and entropy inequalities for quantum Markov semigroups with detailed balance," Journal of Functional Analysis 273, 1810 - 1869 (2017).

[19] Eric A. Carlen and Jan Maas, "Non-commutative calculus, optimal transport and functional inequalities in dissipative quantum systems," (2018), arXiv:1811.04572 [math.OA].

[20] Leonard Gross, "Logarithmic Sobolev inequalities," American Journal of Mathematics 97, 1061 (1975). 
[21] Leonard Gross, "Hypercontractivity and logarithmic Sobolev inequalities for the Clifford-Dirichlet form," Duke Mathematical Journal 42, 383-396 (1975).

[22] P. Diaconis and L. Saloff-Coste, "Logarithmic Sobolev inequalities for finite Markov chains," The Annals of Applied Probability 6, 695-750 (1996).

[23] F. Otto and C. Villani, "Generalization of an inequality by Talagrand and links with the logarithmic Sobolev inequality," Journal of Functional Analysis 173, 361 - 400 (2000).

[24] Alice Guionnet and B Zegarlinksi, "Lectures on logarithmic Sobolev inequalities," in Séminaire de probabilités XXXVI (Springer, 2003) pp. 1-134.

[25] Maxim Raginsky and Igal Sason, "Concentration of measure inequalities in information theory, communications, and coding," Foundations and Trends in Communications and Information Theory 10, 1-246 (2013), arXiv 1212.4663.

[26] Dominique Bakry, Ivan Gentil, and Michel Ledoux, Analysis and geometry of Markov diffusion operators (Springer, Cham; New York, 2014).

[27] D. Burgarth, G. Chiribella, V. Giovannetti, P. Perinotti, and K. Yuasa, "Ergodic and mixing quantum channels in finite dimensions," New Journal of Physics 15, 073045 (2013).

[28] Yu Cao, Jianfeng Lu, and Yulong Lu, "Exponential decay of Rényi divergence under Fokker-Planck equations," (2018), arXiv:1805.06554 [math.AP].

[29] G. Lindblad, "On the generators of quantum dynamical semigroups," Communications in Mathematical Physics 48, 119-130 (1976).

[30] Vittorio Gorini, Andrzej Kossakowski, and E. C. G. Sudarshan, "Completely positive dynamical semigroups of N-level systems," Journal of Mathematical Physics 17, 821-825 (1976).

[31] E. B. Davies, "Markovian master equations," Communications in Mathematical Physics 39, 91-110 (1974).

[32] Heinz-Peter Breuer and F. Petruccione, The Theory of Open Quantum Systems (Oxford University Press, New York, 2007).

[33] Melchior Wirth, "A noncommutative transport metric and symmetric quantum Markov semigroups as gradient flows of the entropy," (2018), arXiv:1808.05419 [math.OA].

[34] Martin Müller-Lennert, Frédéric Dupuis, Oleg Szehr, Serge Fehr, and Marco Tomamichel, "On quantum Rényi entropies: A new generalization and some properties," Journal of Mathematical Physics 54, 122203 (2013).

[35] Mark M. Wilde, Andreas Winter, and Dong Yang, "Strong converse for the classical capacity of entanglement-breaking and Hadamard channels via a sandwiched Rényi relative entropy," Communications in Mathematical Physics 331, 593-622 (2014).

[36] Milán Mosonyi and Tomohiro Ogawa, "Quantum hypothesis testing and the operational interpretation of the quantum Rényi relative entropies," Communications in Mathematical Physics 334, 1617-1648 (2015).

[37] K. Temme, M. J. Kastoryano, M. B. Ruskai, M. M. Wolf, and F. Verstraete, "The $\chi^{2}$-divergence and mixing times of quantum Markov processes," Journal of Mathematical Physics 51, 122201 (2010).

[38] Salman Beigi, "Sandwiched Rényi divergence satisfies data processing inequality," Journal of Mathematical Physics 54, 122202 (2013).

[39] Alexander Müller-Hermes and Daniel Stilck Franca, "Sandwiched Rényi convergence for quantum evolutions," Quantum 2, 55 (2018).

[40] Dénes Petz, "Quasi-entropies for finite quantum systems," Reports on Mathematical Physics 23, 57-65 (1986).

[41] Koenraad M. R. Audenaert and Nilanjana Datta, " $\alpha$ - $z$-Rényi relative entropies," Journal of Mathematical Physics 56, 022202 (2015).

[42] Eric A Carlen, Rupert L Frank, and Elliott H Lieb, "Inequalities for quantum divergences and the Audenaert-Datta conjecture," Journal of Physics A: Mathematical and Theoretical 51, 483001 (2018).

[43] Mario Berta, Omar Fawzi, and Marco Tomamichel, "On variational expressions for quantum relative entropies," Letters in Mathematical Physics 107, 2239-2265 (2017).

[44] Robert Olkiewicz and Bogusław Zegarlinski, "Hypercontractivity in noncommutative $L_{p}$ spaces," Journal of Functional Analysis 161, 246-285 (1999).

[45] Michael J. Kastoryano and Kristan Temme, "Quantum logarithmic Sobolev inequalities and rapid mixing," Journal of Mathematical Physics 54, 052202 (2013). 
[46] Salman Beigi, Nilanjana Datta, and Cambyse Rouzé, "Quantum reverse hypercontractivity: its tensorization and application to strong converses," (2018), arXiv:1804.10100 [quant-ph].

[47] Alexander Müller-Hermes, Daniel Stilck França, and Michael M. Wolf, "Relative entropy convergence for depolarizing channels," Journal of Mathematical Physics 57, 022202 (2016).

[48] Robert Alicki, "On the detailed balance condition for non-hamiltonian systems," Reports on Mathematical Physics 10, 249-258 (1976).

[49] Franco Fagnola and Veronica Umanità, "Generators of detailed balance quantum Markov semigroups," Infin. Dimens. Anal. Quantum. Probab. Relat. Top. 10, 335-363 (2007).

[50] Franco Fagnola and Veronica Umanità, "Generators of KMS symmetric Markov semigroups on $\mathcal{B}(\mathrm{h})$ symmetry and quantum detailed balance," Commun. Math. Phys. 298, 523-547 (2010).

[51] Cambyse Rouzé and Nilanjana Datta, "Relating relative entropy, optimal transport and Fisher information: a quantum HWI inequality," (2017), arXiv:1709.07437 [quant-ph].

[52] Cambyse Rouzé and Nilanjana Datta, "Concentration of quantum states from quantum functional and transportation cost inequalities," Journal of Mathematical Physics 60, 012202 (2019).

[53] Dominique Bakry and Michel Émery, "Diffusions hypercontractives," in Séminaire de Probabilités XIX 1983/84 (Springer, 1985) pp. 177-206.

[54] Cédric Villani, Optimal transport: old and new (Springer, Berlin, 2009).

[55] Karol Zyczkowski and Wojeciech Slomczynski, "The Monge distance between quantum states," Journal of Physics A: Mathematical and General 31, 9095 (1998).

[56] Karol Zyczkowski and Wojciech Slomczynski, "The Monge metric on the sphere and geometry of quantum states," Journal of Physics A: Mathematical and General 34, 6689 (2001).

[57] François Golse, Clément Mouhot, and Thierry Paul, "On the mean field and classical limits of quantum mechanics," Communications in Mathematical Physics 343, 165-205 (2016).

[58] J. Agredo and F. Fagnola, "On quantum versions of the classical Wasserstein distance," Stochastics 89, 910-922 (2017).

[59] François Golse and Thierry Paul, "Wave packets and the quadratic Monge-Kantorovich distance in quantum mechanics," Comptes Rendus Mathematique 356, 177-197 (2018).

[60] David F. Hornshaw, " $L^{2}$-Wasserstein distances of tracial $W^{*}$-algebras and their disintegration problem," (2018), arXiv:1806.01073 [math.OA].

[61] Yongxin Chen, Wilfrid Gangbo, Tryphon T. Georgiou, and Allen Tannenbaum, "On the matrix Monge-Kantorovich problem," (2017), arXiv:1701.02826 [math.FA].

[62] Y. Chen, T. T. Georgiou, and A. Tannenbaum, "Matrix optimal mass transport: A quantum mechanical approach," IEEE Transactions on Automatic Control 63, 2612-2619 (2018).

[63] Yongxin Chen, Tryphon T. Georgiou, and Allen Tannenbaum, "Wasserstein geometry of quantum states and optimal transport of matrix-valued measures," in Emerging Applications of Control and Systems Theory, edited by Roberto Tempo, Stephen Yurkovich, and Pradeep Misra (Springer International Publishing, Cham, 2018) pp. 139-150.

[64] Jean-David Benamou and Yann Brenier, "A computational fluid mechanics solution to the MongeKantorovich mass transfer problem," Numerische Mathematik 84, 375-393 (2000).

[65] Manh Hong Duong, Mark A. Peletier, and Johannes Zimmer, "Conservativedissipative approximation schemes for a generalized Kramers equation," Mathematical Methods in the Applied Sciences 37, 2517-2540 (2014).

[66] Cédric Villani, "Hypocoercivity," Memoirs of the American Mathematical Society 202 (2009). 